\title{
Compartment-dependent chromatin interaction dynamics revealed by liquid chromatin Hi-C
}

Houda Belaghzal ${ }^{1^{*}}$, Tyler Borrman ${ }^{2^{*}}$, Andrew D. Stephens ${ }^{3}$, Denis L. Lafontaine ${ }^{1}$, Sergey V. Venev ${ }^{1}$, Zhiping Weng ${ }^{2}$, John F. Marko ${ }^{3,4}$, Job Dekker ${ }^{1,5,6 \#}$

${ }^{1}$ Program in Systems Biology, Department of Biochemistry and Molecular Pharmacology, University of Massachusetts Medical School, Worcester, MA, 01605, USA

2 Program in Bioinformatics and Integrative Biology, University of Massachusetts Medical School, Worcester, MA, 01605, USA

${ }^{3}$ Department of Molecular Biosciences, Northwestern University, Evanston, IL 60208, USA

${ }^{4}$ Department of Physics and Astronomy, Northwestern University, Evanston, IL 60208, USA

${ }^{5}$ Howard Hughes Medical Institute, Chevy Chase, MD 20815, USA

${ }^{6}$ Lead Contact

* These authors contributed equally

\# Correspondence: Job Dekker (Job.Dekker@umassmed.edu)

\section{Keywords}

Chromosome compartmentalization, nuclear organization, phase separation, microphase, copolymer, nuclear body, chromatin dynamics, chromatin looping

\section{Highlights}

- Liquid chromatin Hi-C detects chromatin interaction dissociation rates genome-wide

- Chromatin conformations in distinct nuclear compartments differ in stability

- Stable heterochromatic associations are major drivers of chromatin phase separation

- CTCF-CTCF loops are stabilized by encirclement of loop bases by cohesin rings

\section{Figures 1-7}

\section{Methods}

\section{Supplemental Figures S1-S6}

Supplemental Tables S1-S3

Supplemental Movies S1-S2. 


\section{SUMMARY}

Chromosomes are folded so that active and inactive chromatin domains are spatially segregated. Compartmentalization is thought to occur through polymer phase/microphase separation mediated by interactions between loci of similar type. The nature and dynamics of these interactions are not known. We developed liquid chromatin $\mathrm{Hi}-\mathrm{C}$ to map the stability of associations between loci. Before fixation and $\mathrm{Hi}-\mathrm{C}$, chromosomes are fragmented removing the strong polymeric constraint to enable detection of intrinsic locus-locus interaction stabilities. Compartmentalization is stable when fragments are over 10-25 kb. Fragmenting chromatin into pieces smaller than $6 \mathrm{~kb}$ leads to gradual loss of genome organization. Dissolution kinetics of chromatin interactions vary for different chromatin domains. Lamin-associated domains are most stable, while interactions among speckle and polycomb-associated loci are more dynamic. Cohesin-mediated loops dissolve after fragmentation, possibly because cohesin rings slide off nearby DNA ends. Liquid chromatin Hi-C provides a genome-wide view of chromosome interaction dynamics. 


\section{INTRODUCTION}

The spatial organization of chromosomes plays roles in many aspects of genome function, including gene regulation, DNA replication, DNA repair, and chromosome compaction and segregation. Genomic and imaging approaches are producing high-resolution descriptions of the conformation of chromosomes in cell populations, in single cells, across the cell cycle, and during development (Bickmore and van Steensel, 2013; Bonev and Cavalli, 2016; Chen et al., 2018; Dekker et al., 2017; Dekker and Mirny, 2016; Gibcus et al., 2018; Hug et al., 2017; Kaaij et al., 2018; Lieberman-Aiden et al., 2009; Nagano et al., 2013; Nagano et al., 2017; Naumova et al., 2013; Nir et al., 2018; Ramani et al., 2017; Rao et al., 2014; Wang et al., 2016).

At the scale of the nucleus, chromosomes occupy separate territories with some interdigitation where they touch (Branco and Pombo, 2006; Cremer and Cremer, 2001). At the mega-base $(\mathrm{Mb})$ scale, different types of chromosomal domains can be discerned. Domains of silent heterochromatin are peripherally localized near the nuclear lamina, and around the nucleolus (Bickmore and van Steensel, 2013; Guelen et al., 2008; Padeken and Heun, 2014). Other domains of silent chromatin such as those bound by polycomb complexes can cluster together as well, e.g. to form polycomb bodies (Pirrotta and Li, 2012). Active segments of the genome are found around bodies such as nuclear speckles (Fakan and Puvion, 1980; Spector and Lamond, 2011) and structures enriched in transcription regulatory factors (Cho et al., 2018; Chong et al., 2018; Iborra et al., 1996; Pombo et al., 1999).

$\mathrm{Hi}-\mathrm{C}$ data confirmed the spatial segregation of active and inactive segments of chromosomes: chromatin interaction maps display a "plaid" pattern, which reflects the segregation of the genome in two major spatial compartments referred to as $A$ and $B$ compartments that correspond to open and active chromatin and closed and silent chromatin, respectively (Lieberman-Aiden et al., 2009; Simonis et al., 2006). High-resolution (kb) Hi-C maps allowed splitting these two major compartments in 5 subtypes (A1, A2, B1, B2, and B3) that differ in interaction patterns and chromatin modification status (Rao et al., 2014). In mammalian cells these domains are typically hundreds of $\mathrm{kb}$ up to several $\mathrm{Mb}$ in size. Belmont and colleagues related these sub-compartments to structures such as speckles (A1 domains) in the interior and the peripheral lamina (B2 and B3) (Chen et al., 2018). Data obtained with SPRITE showed similar clustering of genomic sub-compartments around the nucleolus, speckles, and the periphery (Quinodoz et al., 2018). 
At the scale of hundreds of $\mathrm{kb}, \mathrm{Hi}-\mathrm{C}$ and $5 \mathrm{C}$ experiments uncovered topologically associating domains (TADs; (Dixon et al., 2012; Nora et al., 2012)). TADs were identified as self-interacting domains separated by boundaries that are in many cases bound by CTCF. Higher resolution HiC (Rao et al., 2014), ChIA-PET (Tang et al., 2015), and 4C data (de Wit et al., 2015; Guo et al., 2015; Vietri Rudan et al., 2015) showed that CTCF sites at boundaries can engage in looping interactions, especially when the sites are in convergent orientation.

Major new questions revolve around the molecular and biophysical processes by which different aspects of chromosome conformation form. Significant progress has been made in developing and testing mechanistic models for TAD and loop formation. The model that currently has most experimental support proposes that TADs and loops form via loop extrusion performed by the cohesin complex (Alipour and Marko, 2012; Fudenberg et al., 2017; Fudenberg et al., 2016; Nasmyth, 2001; Riggs, 1990; Sanborn et al., 2015). In this model, loop extrusion proceeds till the complex encounters a CTCF protein bound to a CTCF site in the convergent orientation. As a result, CTCF-CTCF loops form, and interactions between loci located between convergent CTCF sites are generally increased (TADs). The mechanisms of loop extrusion are not known in detail yet, but may involve the cohesin ring encircling strands of DNA at the bases of loops in a topological or pseudo-topological manner (Fudenberg et al., 2017; Haering et al., 2008; Ivanov and Nasmyth, 2005; Kagey et al., 2010; Srinivasan et al., 2018).

Much less is known about the processes that determine nuclear compartmentalization. Mechanisms of compartmentalization are distinct from the formation of TADs and loops, as mutations in CTCF or cohesin disrupt TADs but not compartmentalization per se (Haarhuis et al., 2017; Nora et al., 2017; Nuebler et al., 2018; Rao et al., 2017; Schwarzer et al., 2017; Wutz et al., 2017). Compartmentalization has been proposed to be the result of polymer phase separation driven by attractions between chromatin domains of the same or similar status (Di Pierro et al., 2016; Erdel and Rippe, 2018; Falk et al., 2019; Jost et al., 2014; Lieberman-Aiden et al., 2009; MacPherson et al., 2018; Michieletto et al., 2016; Nuebler et al., 2018; Shi et al., 2018). Polymer models simulating such attractions can reproduce the plaid pattern characteristic of Hi-C interaction maps (Di Pierro et al., 2016; Falk et al., 2019; Jost et al., 2014; Nuebler et al., 2018). However, the molecular and biophysical basis of these attractions is unknown. Possibly these attractions result from co-association of domains with sub-nuclear bodies that themselves appear to form by a process of liquid-liquid phase separation (e.g. 
(Courchaine et al., 2016; Feric et al., 2016; Larson et al., 2017; Marzahn et al., 2016; Sawyer et al., 2018; Strom et al., 2017; Zhu and Brangwynne, 2015). An example is the interaction between heterochromatic loci driven by multivalent interactions among HP1 proteins and between HP1 proteins and H3K9me3-modified chromatin domains (Larson et al., 2017; Strom et al., 2017).

$\mathrm{Hi}-\mathrm{C}$ contact maps readily reveal global spatial separation of active and inactive chromatin domains, and Hi-C sub-compartments suggest the presence of a number of different types of sub-nuclear neighborhoods. However, Hi-C interaction maps are population-averaged, steadystate datasets and do not reveal the biophysical nature of the interactions that lead to this diverse set of nuclear neighborhoods or the dynamic mobility of loci within these compartments. For instance, $\mathrm{A}$ and $\mathrm{B}$ compartments appear equally prominent in $\mathrm{Hi}-\mathrm{C}$ datasets, but whether the forces that lead to their formation are equally strong, frequent, or dynamic is not known.

Live-cell imaging studies have shown that loci are constrained in their motion and that there are substantial variations in the dynamics and mobility of different loci, e.g. euchromatic vs. heterochromatic loci and loci tethered to the nuclear periphery vs. loci located in the nuclear interior (Bronshtein et al., 2009; Bronshtein et al., 2015; Hediger et al., 2002; Marshall et al., 1997; Nagashima et al., 2019; Shinkai et al., 2016; Thakar et al., 2006; Therizols et al., 2010). Such differences can be reproduced in coarse-grained simulations of chromatin (Liu et al., 2018). Imaging-based studies have been instrumental in uncovering aspects of chromatin interactions and dynamics, but are limited in scale, i.e. only one or a few specific loci can be studied at one time. In addition, when whole genome dynamics is analyzed microscopically (e.g. (Zidovska et al., 2013)), positions of specific sequences have not as yet been determined.

New approaches are required to identify and quantify the molecular processes and biophysical forces that drive chromosome and nuclear compartmentalization and to characterize the dynamics and mobility of loci within these compartments. Here we describe liquid chromatin Hi$\mathrm{C}$, a $\mathrm{Hi}-\mathrm{C}$ variant that quantifies the stability of chromosome conformation and chromatin interactions between loci genome-wide. We find that different types of nuclear subcompartments differ in stability of chromatin interactions. The results suggest that compartmentalization is mainly due to strong and stable heterochromatic interactions, while associations between open regions at and around nuclear speckles, and between loci in the B1 sub-compartment enriched in polycomb binding, are more dynamic. 


\section{RESULTS}

\section{Measuring the stability of chromatin interactions that maintain nuclear compartmentalization}

The formation of spatially segregated heterochromatic and euchromatic domains can be viewed as microphase separation of a polymer composed of different types of monomers (loci). For instance, a "block copolymer" is a polymer that contains a series of alternating blocks (e.g., Atype and B-type, or blocks of euchromatin and heterochromatin), each composed of multiple monomers (A monomers and B monomers; Figure 1A). When As attract As and Bs attract Bs, such polymer can fold into spatially segregated domains of As and Bs (Figure 1A; (de Gennes, 1979; Leibler, 1980; Matsen and Schick, 1994)). A key point is that the A and B blocks of a copolymer cannot macroscopically phase separate because they are connected: copolymers undergo microphase separation, forming microdomains of sizes comparable to the polymer coil size of the individual blocks. Applied to chromatin in vivo, microphase separation may underlie the formation of chromatin interaction domains. However, the biophysical forces and interaction dynamics that determine chromosome compartmentalization in cells are not known.

Whether microphase separation of a block copolymer occurs depends on the interaction strengths between monomers as well as the lengths of the blocks of monomers of each type (Figure 1A). Flory-Huggins polymer theory predicts that spatial segregation will occur when the product of the length of the blocks ( $N$, the number of monomers that make up blocks) and their effective preferential homotypic interaction strength $(\chi$, a parameter that represents the difference in the strength of homotypic interactions as compared to heterotypic (A-B) interactions) is larger than a critical value $C$ (de Gennes, 1979; Leibler, 1980; Matsen and Schick, 1994); see Supplemental Materials). Large blocks of a polymer can spatially segregate even when attractive interactions among monomers are weak, while short blocks will only phase separate when interactions are sufficiently strong. Given that in mammals, domains of heterochromatin and euchromatin are typically large (hundreds of kb up to several $\mathrm{Mb}$ ), spatial segregation can occur even when attractive forces between monomers (loci) are weak. Thus, a wide range of interaction strengths between loci within $A$ and $B$ compartments could lead to a spatially segregated genome. The corollary is that observation of spatially compartmentalized chromosomes per se, e.g., by $\mathrm{Hi}-\mathrm{C}$, does not provide quantitative information about the strength of the interactions that drive this organization. Should quantitative measurements of chromatin interaction strength or stability be available genome-wide, such data would allow deeper 
Figure 1

A

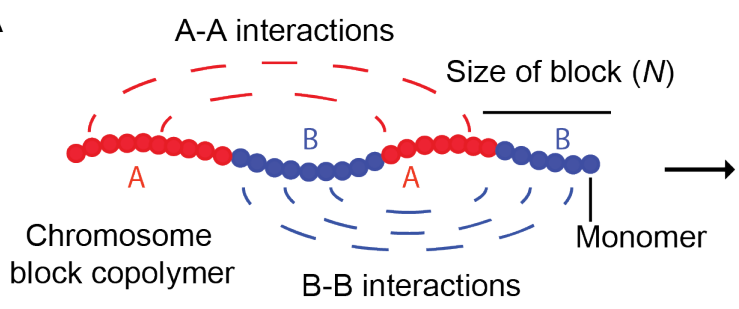

B

Purified nuclei Low density / unstable interactions

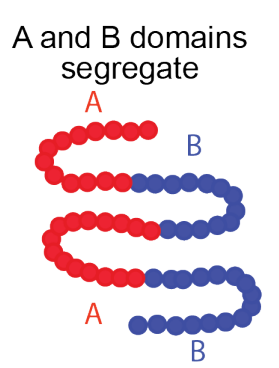

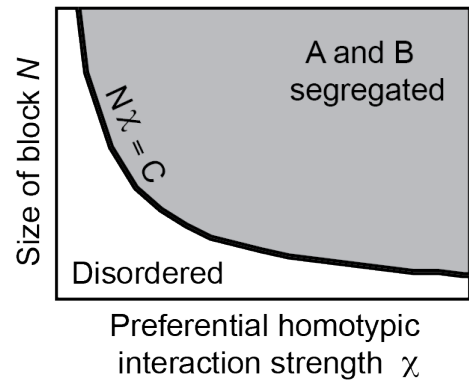

interaction strength $\chi$

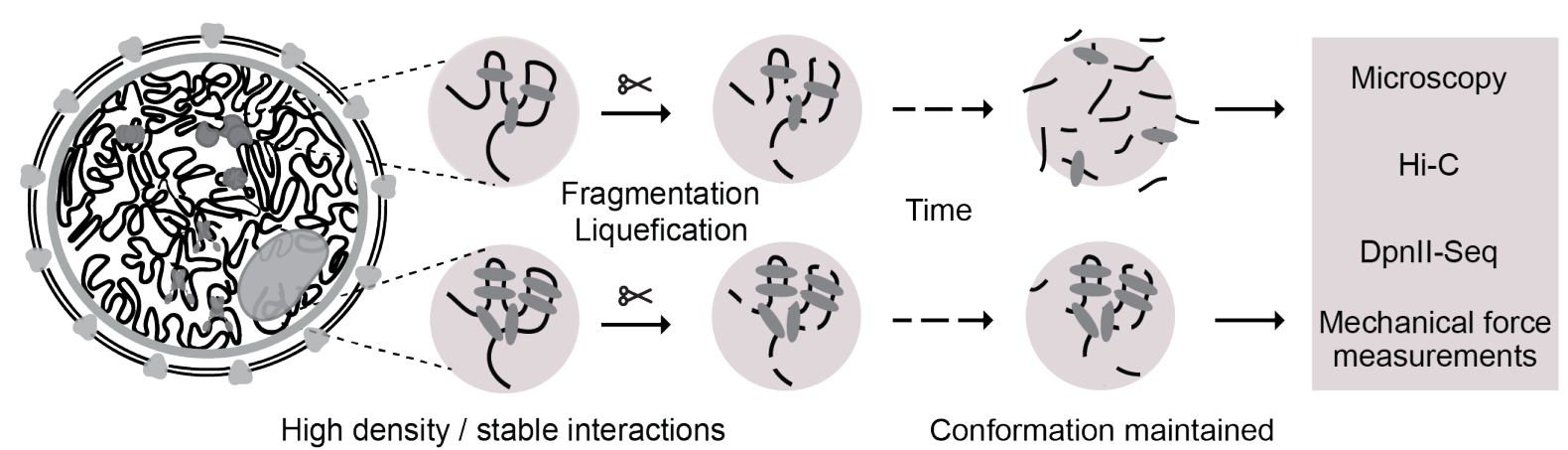

Figure 1: Approach for measuring chromatin interaction stability

A: Block copolymer composed of a series of alternating A and B blocks, each composed of a number of monomers (left). The polymer can fold into spatially segregated domains of As and Bs (middle). FloryHuggins polymer theory predicts that spatial segregation will occur when the product of the length of the blocks $N$ (the number of monomers that make up blocks) and their effective preferential homotypic interaction strength $\chi$ (difference in the strength of homotypic interactions as compared to heterotypic (AB) interactions) is larger than a critical value $C$.

B: Workflow to determine the stability of chromatin interactions genome-wide, DNA (black), varying chromatin features or proteins maintaining DNA conformation (grey ovals).

molecular understanding of the mechanisms leading to chromosome and nuclear compartmentalization, by relating these measurements to binding of specific factors, association with sub-nuclear structures, and the presence of chromatin modifications and processes such as transcription and replication. 
The dependence of microdomain formation on the product of block size and interaction strength suggests an experimental approach to quantify the strengths and dynamics of interactions between individual loci that drive chromosome compartmentalization (Figure 1A, B). One can start with a compartmentalized state of the genome and fragment the chromosomes, e.g., by in situ restriction digestion, and then identify conditions where chromatin fragments become so short that the chromatin interaction strength between the segments is not sufficient to maintain a phase- or microphase-separated state. As a result, chromosomal domains and compartments will disassemble over time and the chromosomal fragments of different type (e.g., As and Bs) will become mixed, i.e. chromatin becomes liquid-like as opposed to a glassy state for intact chromosomes (Shi et al., 2018). The kinetics of this dissolution and mixing process can then be assessed genome-wide by $\mathrm{Hi}-\mathrm{C}$ at different times after chromatin fragmentation. Domains formed by strong, stable, and abundant interactions will dissociate more slowly than domains formed by weak, unstable, or infrequent interactions (Figure 1A, B). Such approach will identify the minimum length of the blocks of monomers required for phase separation, the strength and stability of chromatin interactions, the dissolution kinetics of initially phase-separated subnuclear domains upon fragmentation, and how these parameters vary along the genome. Here we describe such strategy that we call liquid chromatin $\mathrm{Hi}-\mathrm{C}$.

\section{Chromosome conformation in isolated nuclei}

To facilitate enzymatic fragmentation of chromosomes, we isolated nuclei from K562 cells, a cell line with extensive public data on chromosome conformation and chromatin state through efforts of the ENCODE project (ENCODE-Project-Consortium, 2012). We performed four analyses to demonstrate that chromosome conformation in isolated K562 nuclei was the same as that in intact cells. First, DAPI staining and imaging showed that nuclei were intact with Lamin A as a ring at the nuclear periphery (Figure 2A). Second, we performed 3C (Dekker et al., 2002) to assess known looping interactions between the beta-globin locus control region and the expressed gamma-globin genes (Chien et al., 2011; Dostie et al., 2006). These interactions were readily detected in purified nuclei, as they were in intact cells (Figure S1). Third, 5C analysis (Dostie et al., 2006) of a $1 \mathrm{Mb}$ region surrounding the beta-globin locus showed that known CTCF-mediated interactions were also preserved (Figure S1; (Dostie et al., 2006; Kang et al., 2017; Splinter et al., 2006; Tolhuis et al., 2002). Fourth, genome-wide Hi-C analysis (Belaghzal et al., 2017; Lieberman-Aiden et al., 2009) confirmed that chromosome territories, compartments (determined by principle component analysis, with compartments captured by the first principle component (PC1 (Belaghzal et al., 2017; Lieberman-Aiden et al., 2009)), TADs, 
and CTCF-CTCF loops were intact in isolated nuclei and quantitatively similar to those in intact cells (Figure S1). We conclude that chromosome conformation and nuclear compartmentalization as detected by $3 \mathrm{C}$-based assays are intact in purified nuclei.

\section{Extensive chromatin fragmentation leads to the formation of liquid chromatin}

Next, we determined the effect of different levels of chromatin fragmentation on overall nuclear organization. We incubated purified nuclei for four hours with restriction enzymes that digest chromatin with different frequencies. To quantify the extent of digestion, DNA was purified, and the size distribution of DNA fragments was determined using an Agilent Fragment Analyzer. Digestion with HindlII resulted in fragments that ranged in size from $\sim 10-25 \mathrm{~kb}$ (Figure 2B). A minority of molecules was over $25 \mathrm{~kb}(<15 \%)$, indicating that most of the genome was fragmented to a similar extent. Digestion with Dpnll resulted in fragments that ranged in size between $\sim 1$ and $\sim 6 \mathrm{~kb}$, with less than $6 \%$ of fragments $>6 \mathrm{~kb}$ (Figure 2B). Microscopic inspection of nuclear morphology by DAPI and Lamin A immunofluorescence staining showed that fragmentation of chromatin with HindlII had only minor effects on nuclear morphology (Figure 2A). We did occasionally notice some small amounts of DNA emerging as tiny droplets from the nuclear periphery, suggesting that Hindlll digestion led to some solubilization of chromatin. In contrast, fragmentation of chromatin with Dpnll led to large-scale alteration of nuclear morphology as detected by DAPI staining, and large droplets of apparently liquid chromatin (not surrounded by Lamin A) emerged from the nuclear periphery (Figure 2A, arrow).

We next tested whether different chromatin fragmentation levels had an effect on nuclear stiffness, which reflects the integrity of chromosome conformation and chromatin interactions inside the nucleus. We previously showed that single-nucleus isolation and whole-nucleus extension via micromanipulation with micropipettes provides a reliable and robust way to measure the stiffness of the nucleus (Stephens et al., 2017). K562 cells were pre-treated with the actin depolymerizing drug latrunculin $A$ to allow isolation of the nucleus. Isolated nuclei were attached to two micropipettes at opposite ends, and the whole nucleus was extended by moving an extension micropipette. The deflection of a force micropipette multiplied by its premeasured 
Figure 2

A

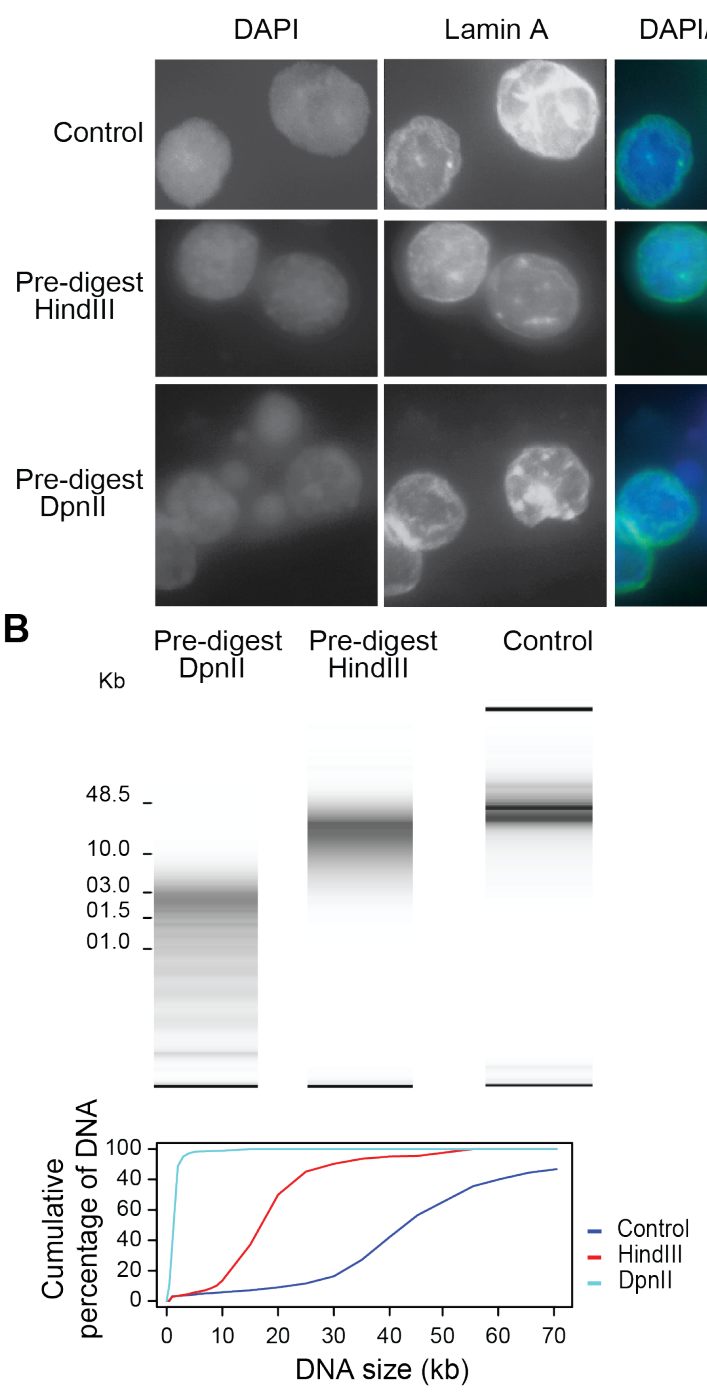

C

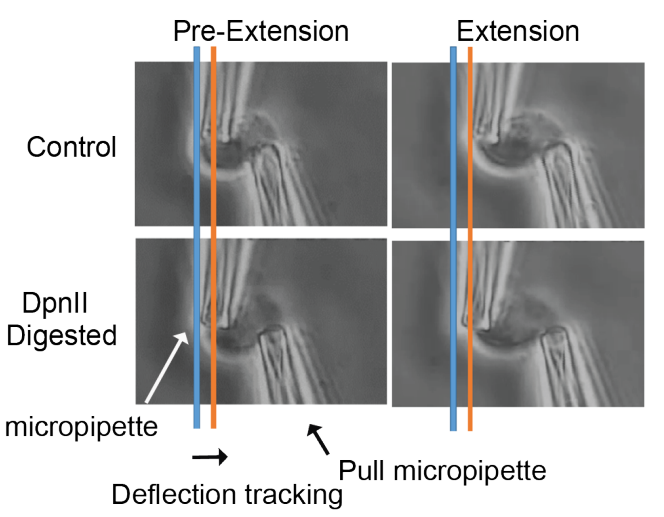

D
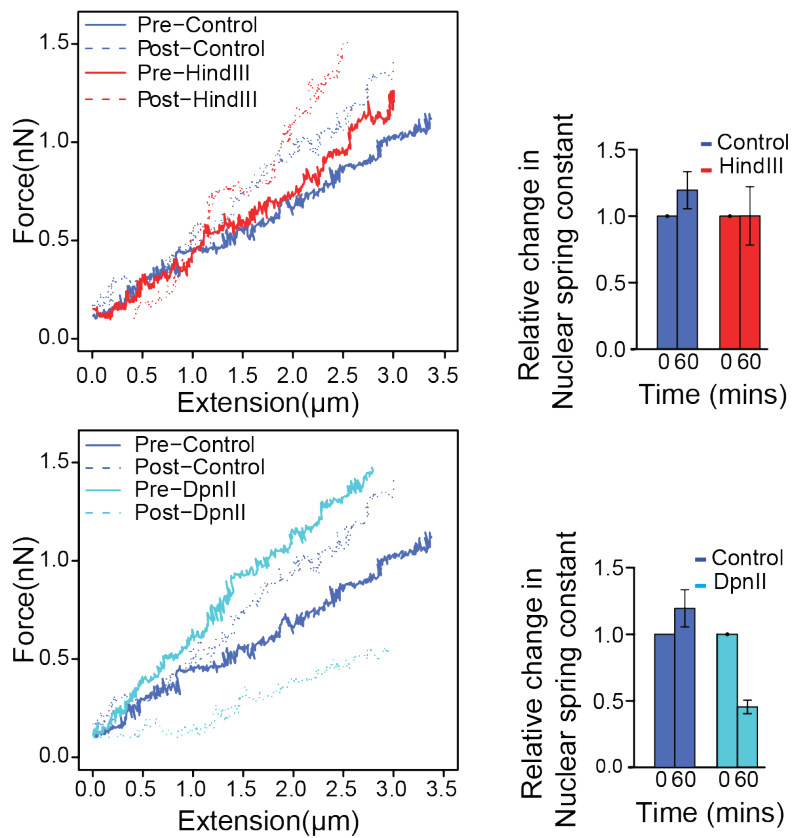

Figure 2: Extensive fragmentation of chromatin leads to liquefied chromatin

A: Nuclear and chromatin morphology before and after chromatin fragmentation. Top row: control nuclei in restriction buffer, middle row nuclei digested for 4 hours with Hindlll. Bottom row: nuclei digested for 4 hours with DpnII. Nuclei were stained with DAPI (left column), with antibodies against Lamin A (middle column). The right column shows the overlay of the DAPI and Lamin A stained images. HindllI digestion did not lead to major alteration in nuclear morphology and chromatin appearance, while Dpnll digestion led to the appearance of DAPI stained droplets (arrow) exiting the nuclei. 
B: Top: DNA purified from undigested nuclei, and nuclei pre-digested with Dpnll and HindIII was run on a Fragment Analyzer. Bottom: cumulative DNA length distributions calculated from the Fragment Analyzer data.

C: Micromanipulation of single nuclei. Isolated nuclei were attached to two micropipettes at opposite ends. Nuclei were extended by moving the right micropipette (Extension micropipette) and the force required was calculated from the deflection of the calibrated "force" (left) pipette. Blue and orange lines indicate the position of the force pipette before and after extension for control nuclei. After digestion of nuclei with Dpnll (bottom) extension required less force as indicated by the much smaller deflection of the force pipette as compared to control nuclei (see also Supplemental Movies 1 and 2).

D. Force-extension plots (left) for control nuclei before and 60 minutes after incubation in restriction buffer (pre- and post control), for nuclei before and after digestion with Dpnll, and for nuclei before and after HindIII digestion. Right panel: relative change in nuclear spring constants, calculated from the slopes of the force-extension plots shown on the left. Bars indicate standard error of the mean $(n=5$ Dpnll predigested nuclei, and $n=4$ HindIII pre-digested nuclei).

spring constant provides a measure of the force (Figure 2C; Supplemental Movie 1, 2). This data provides a force vs. extension plot (Figure 2D, plots on the left), in which the slope of the line fitted to the data is the nuclear spring constant in $\mathrm{nN} / \mu \mathrm{m}$ (Figure 2D, bar plots on the right). Extension between $0-30 \%$ strain measures the chromatin-dominated regime of nuclear force response (Stephens et al., 2017; Stephens et al., 2018), and simulations of nuclear mechanics suggest chromosomal interactions contribute to this regime (Banigan et al., 2017). Isolated single nuclei were measured for their native spring constant before treatment with a restriction endonuclease and then re-measured 60 minutes post-treatment. Nuclei treated with control conditions (only restriction buffer added to the media) showed a slight stiffening of the nucleus (Figure 2D). Treatment of nuclei with HindIII did not significantly decrease the stiffness compared to controls. In sharp contrast, Dpnll-treated nuclei displayed a significant decrease $(\sim 75 \%)$ in stiffness, consistent with previous experiments treating nuclei isolated from mouse embryonic fibroblasts with Alul (Stephens et al., 2017). We conclude that global chromosome and nuclear organization can tolerate genome-wide fragmentation to 10-25 kb segments, indicating that sufficient numbers of relatively stable chromatin interactions are maintained between these large fragments throughout the genome to maintain nuclear stiffness. In contrast, fragmenting the genome to smaller than $6 \mathrm{~kb}$ segments results in extensive loss of chromatin 
morphology, loss of chromatin-mediated stiffness, and the appearance of a liquid-like state of chromatin.

\section{Liquid chromatin Hi-C analysis reveals that compartmental segregation requires chromatin fragments larger than 6 kb}

To determine how chromosome folding and nuclear compartmentalization across the genome is altered as a function of chromatin fragmentation level, we applied $\mathrm{Hi}-\mathrm{C}$ before (conventional $\mathrm{Hi}$ $\mathrm{C}$ ) and after chromatin liquefication (liquid chromatin $\mathrm{Hi}-\mathrm{C}$ ). Nuclei were digested with either HindIII or Dpnll for 4 hours followed by formaldehyde fixation and $\mathrm{Hi}-\mathrm{C}$ analysis (Figure S2A). Liquid chromatin $\mathrm{Hi}-\mathrm{C}$ interaction maps obtained from nuclei that were pre-digested with HindIII were remarkably similar to those obtained with nuclei that were not pre-digested (Figure 3A). The relationship between interaction frequency and genomic distance was largely unaffected, with only a slight redistribution to longer-range interactions (Figure 3B). The ratio of intra- vs. inter-chromosomal interactions was also highly similar to that in untreated nuclei (Figure 3B). Compartments (Figure 3D) were readily detectable as a plaid pattern in Hi-C interaction maps. This pattern is captured by the first principle component (PC1). Compartment positions were unaffected (Spearman $\rho=0.99)$.

Chromosome compartment strength can be visualized and quantified by plotting interaction frequencies between pairs of $40 \mathrm{~kb}$ loci arranged by their values along the first eigenvector (PC1) to obtain compartmentalization saddle plots (Figure $3 \mathrm{C}$ ). In these plots the upper left quadrant represents $B-B$ interactions and the lower right corner represents $A-A$ interactions. Interestingly, in nuclei pre-digested with HindIII, the strength of preferential A-A and B-B interactions (Figure 3C; Supplemental Figure 2C shows a replicate) was somewhat increased, indicating stronger segregation of $A$ and $B$ compartments. This observation, puzzling at first, is in fact readily understood when chromosomes fold as block co-polymers. Polymer theory predicts that very mild fragmentation of a copolymer can enhance phase (or microphase) separation by removing covalent linkages between $A$ and $B$ blocks as long as the fragments are still large enough to sufficiently attract each other (See Methods). Our results show that chromatin fragments of $10-25 \mathrm{~kb}$ are long enough to allow stable segregation of $A$ and $B$ compartments genome-wide. 
bioRxiv preprint doi: https://doi org/10.1101/704957: this version posted July 16,2019 . The copyright holder for this preprint (which was not certified by peer review) is the author/funder, who has granted bioRxiv a license to display the preprint in perpetuity. It is made available under aCC-BY-NC-ND 4.0 International license.

Figure 3

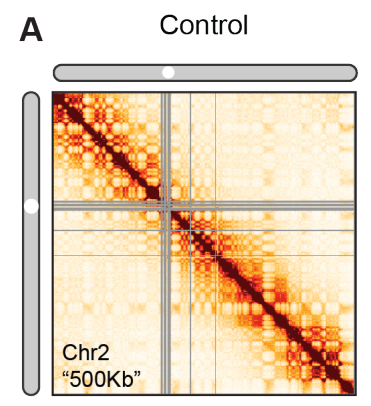

C

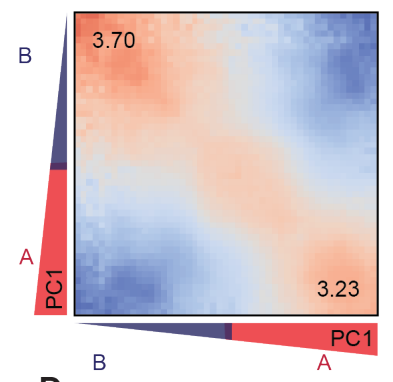

D
Predigest - HindIII
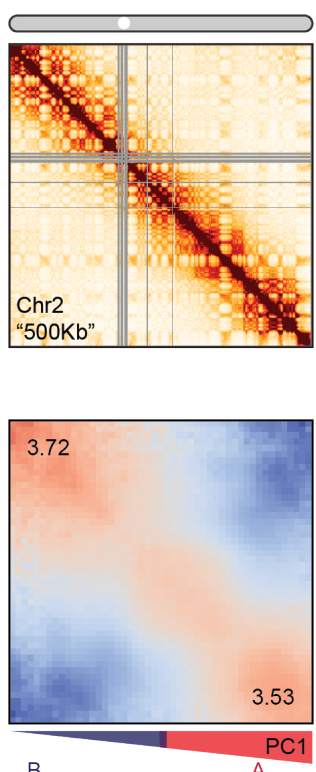

A

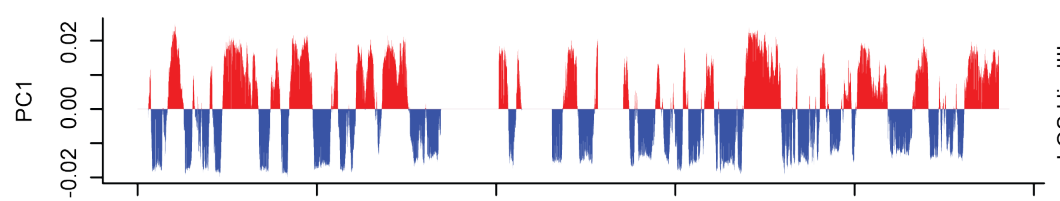
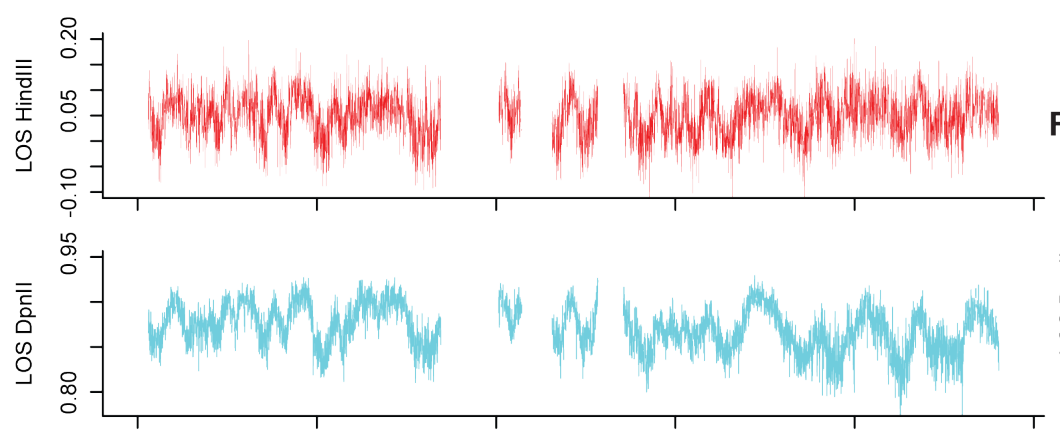

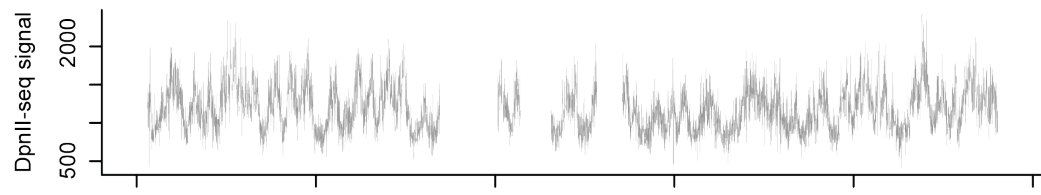

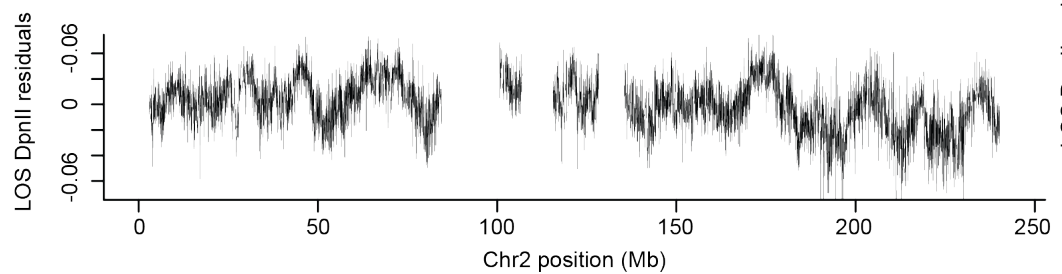

B
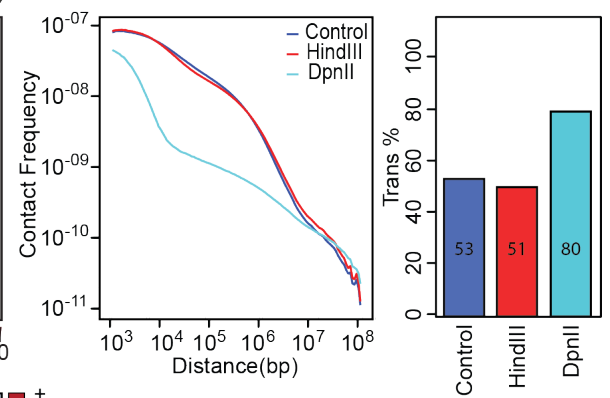

A compartment
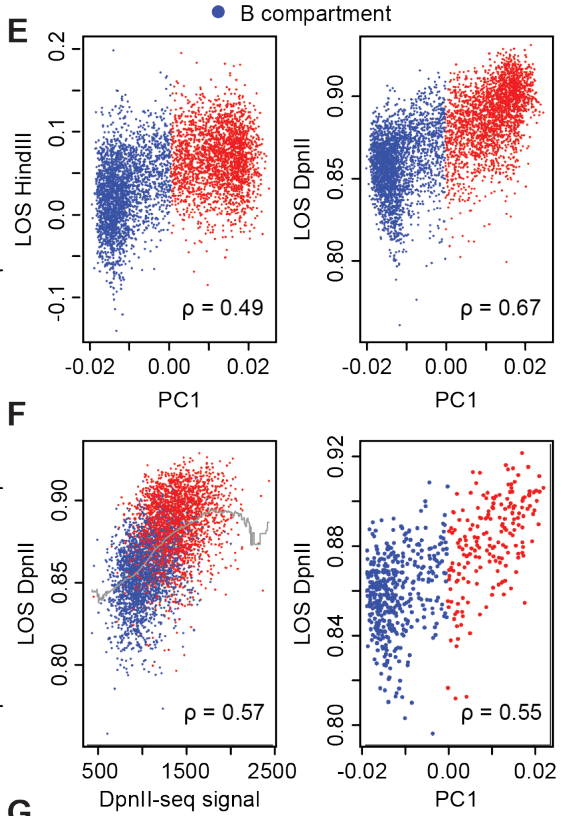

G
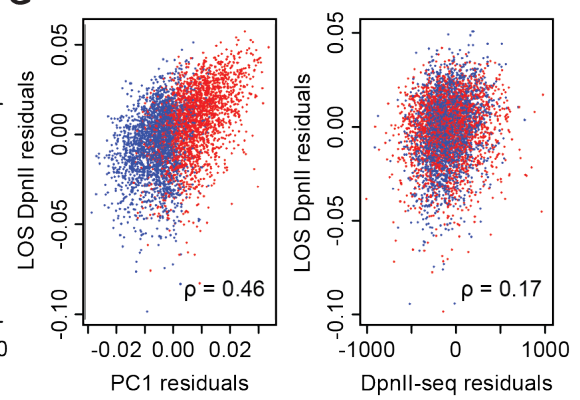
Figure 3: Hi-C analysis reveals chromosome disassembly upon chromatin liquefication

(A) $\mathrm{Hi}-\mathrm{C}$ interaction maps of chromosome 2 binned at $500 \mathrm{~kb}$. Left: interaction map for control nuclei in restriction buffer prior to pre-digestion. Middle: nuclei pre-digested for 4 hours with HindIII prior to Hi-C. Right: nuclei digested for 4 hours with Dpnll prior to Hi-C (see Figure S2A).

(B) Left: genome-wide interaction frequency as function of genomic distance for control nuclei (dark blue), nuclei pre-digested with HindIII (red), and nuclei pre-digested with Dpnll (cyan). Right: percentage of inter-chromosomal (trans) interaction frequencies.

(C) Compartmentalization saddle plots: average intra-chromosomal interaction frequencies between 40 $\mathrm{kb}$ bins, normalized by expected interaction frequency based on genomic distance. Bins are sorted by their PC1 value derived from $\mathrm{Hi}-\mathrm{C}$ data obtained with control nuclei. In these plots preferential $\mathrm{B}-\mathrm{B}$ interactions are in the upper left corner, and preferential A-A interactions are in the lower right corner. Numbers in corners represent the strength of $A A$ interactions as compared to $A B$ interaction and $B B$ interactions over BA interactions (Figure S4B).

(D) Top plot: Eigenvector 1 values (PC1, $40 \mathrm{~kb}$ resolution) across a section of chromosome 2, representing A (red) and B (blue) compartments. Second plot: Loss of pair-wise interactions "LOS" (Methods and Figure S2B) along chromosome 2 at $40 \mathrm{~kb}$ resolution for nuclei pre-digested with HindIII. Third plot: LOS for nuclei pre-digested with Dpnll. Fourth plot: Dpnll-seq signal along chromosome 2 at $40 \mathrm{~kb}$ resolution. Bottom plot: LOS-residuals for nuclei pre-digested with Dpnll after correction for Dpnll signal.

E) Correlation between LOS for nuclei pre-digested with HindIII (left) or DpnII (right) and PC1 (for chromosome 2, Spearman correlation values are indicated).

F) Left: correlation between LOS for nuclei pre-digested with Dpnll and Dpnll-seq signal (for chromosome 2). Grey line indicates moving average used for residual calculation. Right: correlation between LOS for nuclei pre-digested with Dpnll and PC1 for loci cut to the same extent by Dpnll (1000-1100 Dpnll-seq reads/ $40 \mathrm{~kb}$ bin; for chromosome 2). Spearman correlation values are indicated.

G) Left: partial correlation between residuals of LOS for nuclei pre-digested with Dpnll and residuals of PC1 after correcting for correlations between LOS and Dpnll-seq and PC1 and Dpnll-seq signal. Right: partial correlation between residuals of LOS for nuclei pre-digested with Dpnll and residuals of Dpnll-seq signal after correcting for correlations between LOS and PC1 and Dpnll-seq signal and PC1. Spearman correlation values are indicated.

Much more extensive changes in chromosome conformation were observed when nuclei were pre-digested for 4 hours with the frequent cutting enzyme Dpnll (Figure 3A) followed by formaldehyde fixation and $\mathrm{Hi}-\mathrm{C}$ analysis. We observed a considerable loss in shorter range $(<10$ $\mathrm{Mb}$ ) intra-chromosomal interactions, with a gain of longer range (>10 $\mathrm{Mb}$ ) interactions and inter- 
chromosomal interactions (Figure 3B). The gain in inter-chromosomal interactions appeared to be the result of random mixing of $A s$ and $B s$ as the preference for interchromosomal $A-A$ and $B-$ $\mathrm{B}$ interactions decreased. Moreover, compartment strength in cis was greatly reduced with a greater relative reduction evident in the $\mathrm{A}$ compartment (Figure $3 \mathrm{C}$ ). This more prominent loss of A-A interactions compared to B-B interactions is also apparent from direct visual inspection of the $\mathrm{Hi}-\mathrm{C}$ interaction maps: while in $\mathrm{Hi}-\mathrm{C}$ maps from undigested nuclei a plaid interaction pattern is clearly visible with two alternating patterns of chromatin interactions (representing $A$ and $B$ compartments), in liquid chromatin Hi-C maps from Dpnll-pre-digested nuclei one of these two patterns is weakened more (corresponding to the A compartment). Combined, these observations show that fragmentation in $<6 \mathrm{~kb}$ fragments leads to loss of spatial segregation of $\mathrm{A}$ and $\mathrm{B}$ compartments and dissolution of chromosome conformation genome-wide but with more extensive loss of the A compartment.

\section{Quantification of chromosome conformation dissolution upon chromatin fragmentation}

Loss of chromosome conformation and dissolution of chromosomal compartments will result in random mixing of previously spatially separated loci both in cis and in trans. In $\mathrm{Hi}-\mathrm{C}$ this will be apparent by loss of short-range interactions and gain in longer range and inter-chromosomal interactions. We used this phenomenon to quantify for each locus along the genome the extent of loss of chromosome conformation upon chromatin fragmentation. Specifically, using $\mathrm{Hi}-\mathrm{C}$ data binned at $40 \mathrm{~kb}$ resolution we developed a metric which represents the percentage change in short range intra-chromosomal interactions (up to $6 \mathrm{Mb}$ ) for each fragmentation condition relative to control nuclei, which we call loss of structure (LOS) (Figure S2B).

We first calculated LOS after 4 hours for chromatin fragmented with HindIII. We observe that in general short-range interactions are only somewhat reduced, consistent with the above observation that interactions are somewhat redistributed to longer-range interactions (Figure 3B). When LOS is plotted along chromosomes (Figure 3D), we observed that LOS is not uniform: some regions display more loss of short-range interactions than others. To determine how the loss of interactions is related to $A$ and $B$ compartments, we compared LOS to the PC1 value that captures compartmentalization (Lieberman-Aiden et al., 2009) and observed that LOS was positively correlated with PC1 (Figure 3D, 3E left panel): $\sim 3-12 \%$ loss for loci in A and $<5 \%$ loss for loci in $B$ (Figure $3 \mathrm{E}$ ). We note that this effect is very small and close to technical variation between replicates. Thus, chromosome conformation and 
compartmentalization are largely intact in nuclei pre-digested with HindIII (See Supplemental Figure S2C for a replicate).

We performed the same analysis for nuclei pre-digested with Dpnll for 4 hours followed by formaldehyde fixation and $\mathrm{Hi}-\mathrm{C}$. Consistent with the micromechanical measurements described above, we find an extensive loss of chromosome conformation, with LOS generally $>80 \%$. LOS varies along chromosomes and is strongly positively correlated with PC1 with loci in the A compartment displaying the largest loss (Figure 3D, 3E). These results again show that chromatin fragmentation to $<6 \mathrm{~kb}$ fragments leads to extensive genome-wide dissolution of chromosome conformation, random mixing of loci, and loss of spatial segregation of $A$ and $B$ compartments, with the A compartment affected to the largest extent.

\section{Independent contributions of compartment status and fragmentation level to chromatin dissolution}

As outlined above, phase segregation of polymers is predicted to depend on both the length of fragments and the attractive forces between them. Therefore, one explanation for the greater effect of fragmentation on chromatin interactions and chromosome conformation in the $A$ compartment could be that Dpnll cuts more frequently in the open and potentially more accessible A compartment. To assess this, we determined the cutting frequency of Dpnll in isolated nuclei across the genome (Dpnll-seq; Supplemental Figure S3). Nuclei were digested with Dpnll for 4 hours, and free ends were filled in with biotinylated nucleotides. After shearing, biotin-containing fragments were isolated, DNA was sequenced and reads were mapped to the genome. The frequency of fragments mapping with one end at a Dpnll restriction site was calculated along chromosomes at $40 \mathrm{~kb}$ resolution and compared to PC1. We find that digestion frequency is positively correlated with PC1 and with LOS (Figure 3D, 3F left panel; Figure S2D).

To determine whether the correlation between LOS and PC1 is only due to the fact that DpnII digestion is correlated with PC1 we calculated the partial correlation between LOS and PC1 after correcting for the correlations of PC1 and LOS with Dpnll digestion frequency (see Methods for details). We find that the residuals of PC1 and LOS are still highly correlated (Spearman $\rho=0.46$ for chromosome 2; Figure 3G). To illustrate the correlation between LOS and PC1 independent of fragmentation level directly we selected a set of loci along chromosome 2 that are all cut to the same extent (1000-1100 reads in the Dpnll-seq dataset). 
When we plot LOS vs. PC1 for this set we find a strong correlation (Figure 3F right panel, Spearman $\rho=0.55)$. We also determined the partial correlation between LOS and Dpnll-seq signal after correcting for their correlations with PC1. We find that after this correction LOS and Dpnll-seq signal remain correlated (Spearman $\rho=0.17$, Figure 3G right panel). We conclude that when generally digested to $<6 \mathrm{~kb}$ fragments both compartment status (PC1) and fragment size contribute to LOS. Importantly, this implies that the interaction strength between chromatin segments is related to their compartment status.

\section{Dissociation kinetics of chromatin interactions and compartments}

The observation that pre-digestion of chromatin with Dpnll produces chromatin fragments that are too small to maintain segregation of chromatin in $A$ and $B$ compartments allowed us to measure the dissociation kinetics of compartments and stability of chromatin interactions as loci become mobile and start to mix. We first determined the kinetics of chromatin fragmentation (Figure S4A). We digested nuclei with Dpnll for different amounts of time, ranging from 5 minutes to 16 hours. At each time point, we isolated DNA to determine the extent of digestion (Figure 4A). After 5 minutes the size range of fragments was between 3 and $15 \mathrm{~kb}$ ( $80 \%$ of fragments). After one hour $80 \%$ of DNA fragments were smaller than $7 \mathrm{~kb}$ and during the subsequent hours of digestion the fragments became gradually shorter. After 16 hours of digestion $85 \%$ of fragments were smaller than $3.5 \mathrm{~kb}$. We again sequenced DNA ends to determine the distribution of Dpnll cuts across the genome (Figure 4B). We find that at all timepoints the number of Dpnll cuts per $40 \mathrm{~kb}$ bin was correlated with PC1 (Figure 4B), but that the pattern did not change much over time (Figure 4B, correlation matrix; Figure S5). We conclude that digestion is more frequent in the A compartment at all timepoints, but that the pattern of fragmentation along chromosomes is relatively stable over the timecourse.

Micromanipulation was again used to measure the nuclear spring constant corresponding to nuclear stiffness. Nuclei displayed a significant loss in stiffness within 5 minutes, reaching background levels after 1 hour ((Stephens et al., 2017), Figure 4C) when chromatin fragments are smaller than $7 \mathrm{~kb}$. Combined these analyses show that the bulk of DNA fragmentation and chromatin liquefication occurs within the first hour, after which the nuclei have completely lost their nuclear stiffness. 
bioRxiv preprint doi: https://doi.org/10.1101/704957: this version posted July 16,2019 . The copyright holder for this preprint (which was not certified by peer review) is the author/funder, who has granted bioRxiv a license to display the preprint in perpetuity. It is made available under aCC-BY-NC-ND 4.0 International license.

Figure 4

A

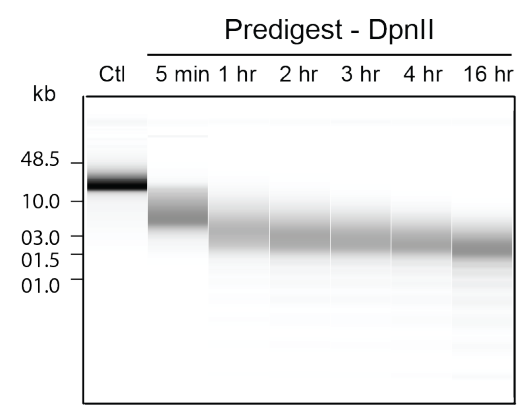

D

\section{B}

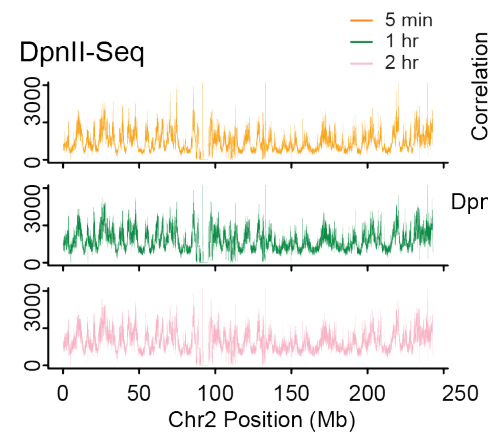

C

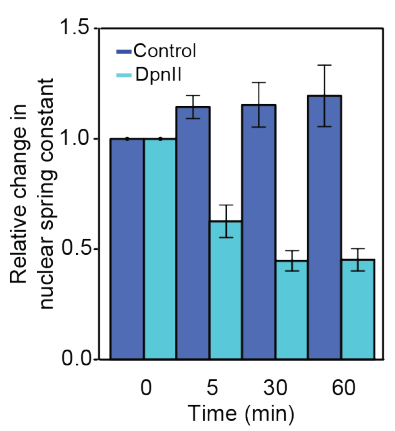

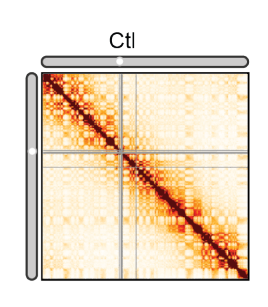
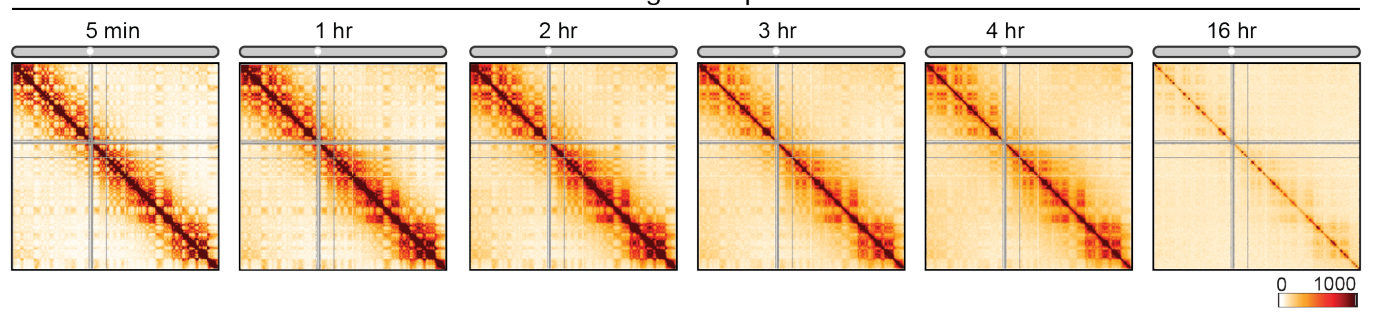

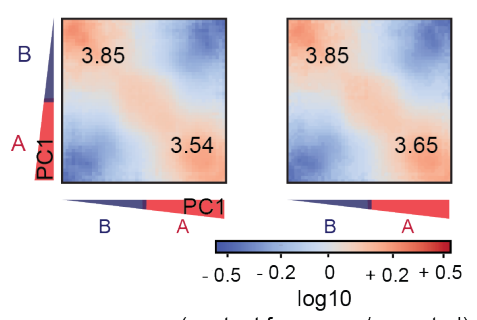

E
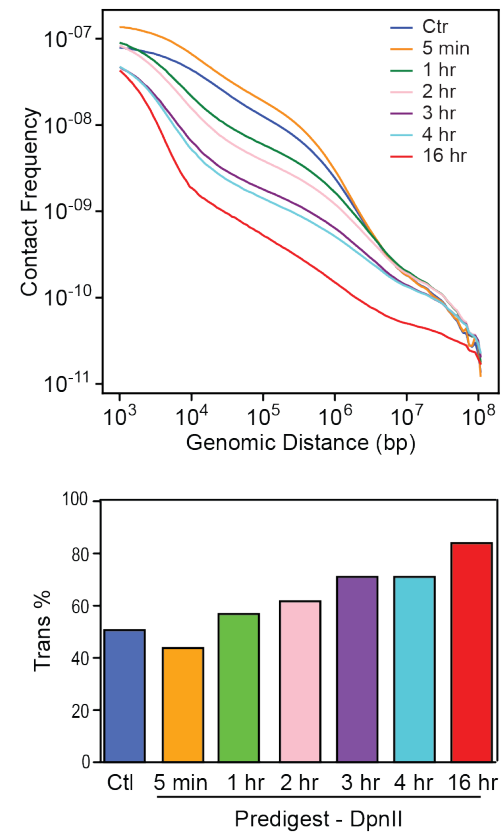
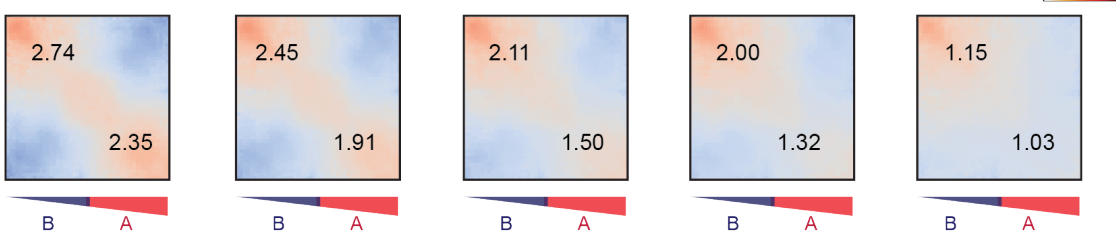

F
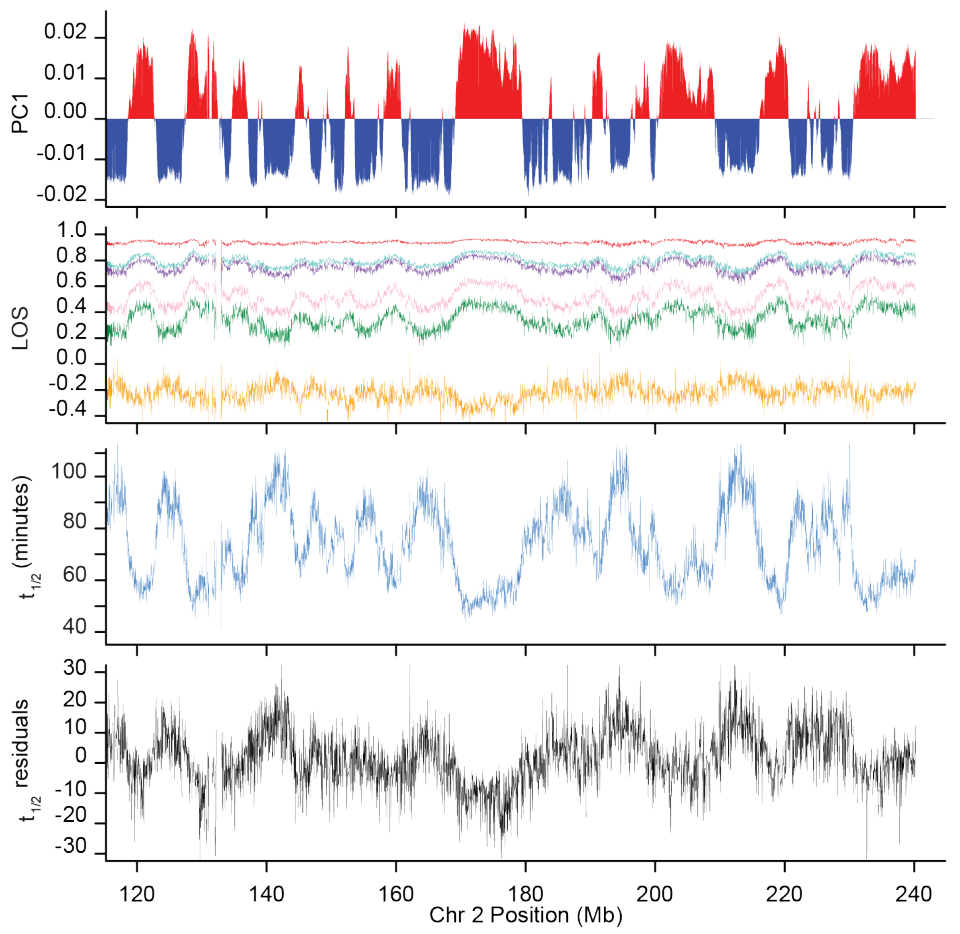
Figure 4: Kinetics of chromatin fragmentation and chromatin dissolution

(A) DNA purified from undigested nuclei, and nuclei pre-digested with Dpnll for different time points were run on a Fragment Analyzer.

(B) Left: Dpnll-seq signals along chromosome 2 binned at $40 \mathrm{~kb}$ resolution after digestion for 5 minutes, 1 hour and 2 hours. Right: correlation between Dpnll-seq signals and PC1 and between Dpnll-seq signals at different time points.

(C) Relative change in nuclear spring constant ( $\mathrm{nN} / \mu \mathrm{m})$ after Dpnll fragmentation at different time points. Spring constant is significantly decreased after 5 minutes and at background level by 1 hour $(p=0.002$, two-tailed t-test).

(D) Top row: Hi-C interaction maps of chromosome 2 binned at $500 \mathrm{~kb}$. Control: nuclei in restriction buffer for 4 hours. Pre-digest Dpnll: nuclei were pre-digested with Dpnll for 5 minutes up to 16 hours. (Figure S4A).

Bottom row: compartmentalization saddle plots for the corresponding conditions. Numbers indicate strength of A-A and B-B interactions.

(E) Top: genome-wide interaction frequency as function of genomic distance for $\mathrm{Hi}-\mathrm{C}$ data shown in panel (D). Bottom: percentage of inter-chromosomal (trans) interactions genome-wide for control nuclei and for nuclei pre-digested with Dpnll for up to 16 hours.

(F) Top: PC1 along a section of $120 \mathrm{Mb}$ of chromosome 2. Second plot: LOS along chromosome 2 at 40 $\mathrm{kb}$ resolution for all time points (Figure S2B). Third plot: half-life $\left(t_{1 / 2}\right)$ values derived from the exponential fit of the six time-points for every $40 \mathrm{~kb}$ bin (Figure S4C). Bottom plot: residuals of $t_{1 / 2}$ after correcting for correlations between $t_{1 / 2}$ and Dpnll-seq (Dpnll-seq data for $t=1$ hour).

Next, we used liquid chromatin $\mathrm{Hi}-\mathrm{C}$ to determine the kinetics with which chromosome conformation and compartmentalization are lost after chromatin fragmentation. Nuclei were predigested with Dpnll for 5 minutes up to 16 hours, followed by formaldehyde fixation and Hi-C analysis (Figure S4A). Interestingly, after 5 minutes of pre-digestion chromosome conformation and compartmentalization are intact, even though chromatin was fragmented to $3-15 \mathrm{~kb}$ segments before fixation and nuclear stiffness was significantly reduced (Figure 4C, D). Further, the percentage of intra-chromosomal interactions especially for loci separated by $<1 \mathrm{Mb}$ was increased (Figure 4E). Compartmentalization is somewhat increased for the A compartment (Figure 4D). These increases may be due to the fact that fragments are on average around 10 kb long, only somewhat shorter than after Hindlll digestion, but longer than after 4 hours of Dpnll digestion (Figures 2, 3). As discussed above mild fragmentation of a block copolymer is predicted to enhance both microphase or phase separation, depending on the cut frequency 
(see Methods). The results also indicate that interactions among fragments of around $10 \mathrm{~kb}$ are strong enough for compartmentalization to occur.

At subsequent time points, when most chromatin fragments are $<7 \mathrm{~kb}$ long and nuclear stiffness is completely lost, we observe increased loss of intra-chromosomal interactions and concomitant increased inter-chromosomal interactions genome-wide (Figure 4D, 4E). Compartmentalization, as quantified by the preference of $A-A$ and $B-B$ interactions over A-B interactions, is progressively lost (Figure 4D, lower row of heatmaps, Figure S4B). A-A interactions disappear faster than B-B interactions. After 16 hours, only a low level of preferential B-B interaction remains. This analysis shows that chromatin fragmentation transiently leads to more frequent intra-chromosomal interactions and stronger compartmentalization due to partial digestion which is then followed by progressive genomewide dissolution of chromosome conformation as fragments become too short, and interactions between these fragments too weak to maintain compartmentalization.

\section{Quantification of the half-life of chromosome conformation across the genome}

To quantify the kinetics of loss of chromosome conformation and compartmentalization, we calculated LOS genome-wide for each time point (Figure 4F). At $t=5$ minutes, LOS is generally negative indicating a gain in chromatin interactions: on average $\sim 25 \%$ gain of intrachromosomal interactions between loci separated by $<6 \mathrm{Mb}$, consistent with the initial increase in overall intra-chromosomal interactions described above (Figure 4E). LOS is inversely correlated with PC1, indicating that loci located within A compartments gain more intrachromosomal interactions than loci located within B compartments (Spearman $\rho=-0.53$ for chromosome 2, Spearman $\rho=-0.49$ genome-wide). At subsequent time points, LOS is increasingly positive as intra-chromosomal interactions are progressively lost and non-specific inter-chromosomal interactions are gained. LOS is the highest for loci located in the $A$ compartment. At $t=16$ hours, LOS is generally as high as $90 \%$, intra-chromosomal interactions are low (<20\% of total), and only preferential B-B interactions are still observed in the Hi-C interaction map (Figure 4D). Very similar results were obtained with an independent replicate of this time course experiment (see below). In that experiment, we again observed an initial transient gain in intra-chromosomal interactions after 5 minutes of pre-digestion, followed by a progressive loss of chromosome conformation genome-wide, but especially in the A compartment. 
Now that we measured LOS as a function of time after chromatin fragmentation, we could calculate the dissociation rates of chromatin interactions genome-wide. LOS as a function of time for each $40 \mathrm{~kb}$ locus was fitted to an exponential curve, which was then used to calculate the time at which each locus lost $50 \%$ of its intra-chromosomal (<6 Mb) interactions (Figure $\mathrm{S} 4 \mathrm{C}$ ). We refer to this time as the half-life $\mathrm{t}_{1 / 2}$ (minutes) of chromatin interactions at each locus (Fig. 4F). A short $\mathrm{t}_{1 / 2}$ represents unstable interactions, while more stably interacting loci will have longer $t_{1 / 2}$ values. Examining $t_{1 / 2}$ along chromosomes, we observe a strong inverse correlation with PC1 (Spearman $\rho=-0.87$; Figure S4F): interactions in the A compartment dissolve relatively fast $\left(t_{1 / 2}=40-80\right.$ minutes) while interactions in the $B$ compartment dissolve slower $\left(t_{1 / 2}\right.$ $=60-120$ minutes; Figure S4D). We also calculated $t_{1 / 2}$ genome-wide for the second independent time course experiment and find a strong correlation between $\mathrm{t}_{1 / 2}$ calculated from the two datasets (Spearman $\rho=0.78$ for chromosome 2; Spearman $\rho=0.76$ genome-wide; Figure S4E). The value of $t_{1 / 2}$ is proportional to the dissociation rate constant and thus independent of the initial level of intra-chromosomal interactions for a given locus. Indeed, $t_{1 / 2}$ remains highly correlated with PC1 even after correcting for correlations between the initial level of intra-chromosomal ( $<6 \mathrm{Mb}$ ) interactions and $t_{1 / 2}$ and between the level of intra-chromosomal interactions and PC1 (Spearman $\rho=-0.82$, Figure S4F, G).

We can also estimate the half-life of compartmentalization directly by calculating the rate of loss of preferential A-A and B-B interactions specifically from the compartmentalization saddle plots shown in Figure 4D. Using this metric, similar values of $t_{1 / 2}$ were observed: $50 \%$ loss of preferential A-A interactions was observed after $\sim 60$ minutes, while a $50 \%$ loss of preferential BB interactions occurred after $\sim 115$ minutes.

\section{Independent contributions of compartment status and fragmentation level to the half-life of chromatin interactions}

Similar to LOS, $t_{1 / 2}$ is correlated with Dpnll digestion frequency at all timepoints (Figure S5A). We again determined the individual contributions of compartment status and fragmentation level to $t_{1 / 2}$. We calculated the partial correlation between $t_{1 / 2}$ and PC1 after correcting for correlations between PC1 and $t_{1 / 2}$ with Dpnll cutting frequency. We find that $t_{1 / 2}$ and PC1 remain strongly correlated (Figure 4F), regardless of which Dpnll fragmentation dataset (genome wide Spearman $\rho$ ranging from -0.41 to $-0.60, t=5$ min up to $t=16$ hours) was used for the calculation of the partial correlation (Figure S5A, S5B). This is illustrated by plotting $t_{1 / 2}$ residuals as a function of Dpnll fragmentation level (Figure S5C). Although loci in the A compartment are 
often cut more frequently than loci in the B compartment, when comparing loci cut with similar frequency, loci in the A compartments had shorter half-lives (Figure S5C). We also calculated the partial correlation between $t_{1 / 2}$ and fragmentation level after correcting for their correlations with PC1 and find that fragmentation level also contributes to $t_{1 / 2}$ (genome wide Spearman $\rho=$ -0.48 , chromosome 2 Spearman $\rho=-0.31$ ). We conclude that after digestion with DpnII dissolution kinetics are determined by both the compartment status of loci and their fragmentation level.

Finally, we considered whether we could have overestimated the $t_{1 / 2}$ for loci in the $B$ compartment because fragmentation of these loci could be slower than for loci in the $A$ compartment. We reasoned that because after 1 hour incubation with Dpnll digestion is largely complete, calculation of LOS using the $\mathrm{Hi}-\mathrm{C}$ data at $\mathrm{t}=1$ hour as starting condition would provide an estimate of dissolution kinetics starting at a timepoint when $A$ and $B$ compartments are both extensively fragmented. We find that LOS, and $t_{1 / 2}$ calculated this way are still strongly correlated with $\mathrm{PC} 1$, and this correlation remains strong after correcting for fragmentation level (Supplemental Figure S5D, S5E, S5F).

\section{Compartment size and boundaries influence chromatin interaction stability}

We next explored the correlation between PC1 and $t_{1 / 2}$ in more detail. Although these two parameters are highly correlated, visual inspection of the data suggested that the largest $A$ compartments appeared to have the smallest $t_{1 / 2}$ while the largest $B$ compartments had the largest $t_{1 / 2}$. To quantify this, we plotted $t_{1 / 2}$ for each $40 \mathrm{~kb}$ bin as a function of the size of the compartment that the bin was located in (Figure S6D). We find that loci within small $A$ compartments had larger $t_{1 / 2}$ values than loci in larger $A$ compartments. Analogously, loci within small $B$ compartments had smaller $t_{1 / 2}$ values than loci in larger $B$ compartments.

One explanation for this effect is that proximity to a compartment boundary modulates the stability of loci. To analyze this, we aggregated PC1 and $t_{1 / 2}$ around A-B compartment boundaries (Figure S6C). We find that while PC1 switches sharply at boundaries, $t_{1 / 2}$ changes less rapidly. As a result, loci in A compartments but near a boundary have larger $t_{1 / 2}$ values than expected for their PC1 value, while loci in B compartments but near a boundary have smaller $t_{1 / 2}$ values than expected for their PC1 value. This boundary effect can contribute to bins in smaller compartments having larger (A compartment) or smaller (B-compartment) $t_{1 / 2}$ values than loci in larger compartments. 


\section{Dissociation kinetics of chromatin interactions at different sub-nuclear structures}

A compartments can be split into A1 and A2 sub-compartments that are both characterized by open and active chromatin, with the $\mathrm{A} 1$ sub-compartment the most enriched in active histone modifications. The A1 sub-compartment is found in close proximity to nuclear speckles (Chen et al., 2018). Inactive chromatin can be classified into B1, B2 and B3 sub-compartments. B2 and B3 are generally inactive domains and are located near the nuclear lamina (B2 and B3) and the nucleolus (B2) (Chen et al., 2018; Quinodoz et al., 2018; Rao et al., 2014). B1 is enriched in the repressive H3K27me3 mark, which is often associated with polycomb binding. To relate subcompartment status to chromatin dissociation rates, we compared the residuals of $t_{1 / 2}$ (after correcting for fragmentation level) for loci located in the 5 sub-compartments defined for K562 cells ((Xiong and $\mathrm{Ma}, 2019)$; Figure $5 \mathrm{~A})$. We find that residual $\mathrm{t}_{1 / 2}$ varies greatly between subcompartments: $t_{1 / 2}(A 1) \sim t_{1 / 2}(B 1)<t_{1 / 2}(A 2)<t_{1 / 2}(B 2) \sim t_{1 / 2}(B 3)$.

It is noteworthy that interaction dissociation rates for loci in the B1 sub-compartment are as high or higher (residuals of $\mathrm{t}_{1 / 2}$ as or more negative) than those for loci in the active and open A1 subcompartment. Many B1 sub-compartments are indeed embedded within A compartments (Figure S6C, E) and a subset is found close to nuclear speckles (Chen et al., 2018). Within the B compartment, interactions between Lamin-associated loci in the B3 sub-compartments dissociate the slowest while interactions between loci in the B2 sub-compartment dissociate somewhat faster. These observations indicate that loci associated with different sub-nuclear structures display a range of interaction stabilities. We also noted that $\mathrm{A} 2$ sub-compartments are frequently found near A-B boundaries (Figure S6C) and that A2 and B2 sub-compartments tend to be located in smaller $A$ and $B$ compartments respectively (Figure S6E), providing an additional explanation for the compartment size effect on $t_{1 / 2}$ described above.

Consistent with the relation between chromatin state, sub-compartment status, and DNA replication timing (Rao et al., 2014), we find a strong correlation between $t_{1 / 2}$ residuals and replication timing. We split the genome into 10 bins, where each bin corresponds to sets of loci that share the same $t_{1 / 2}$ residual interval. We then explored the enrichment for varying chromatin features for each $t_{1 / 2}$ residual interval (Figure 5B, D). Chromatin interactions for early replicating domains had short half-lives, while interactions for loci in later replicating domains were more stable (Figure $5 B$ ). Interestingly, loci with the shortest $t_{1 / 2}$ residuals replicate in the middle of $S$ phase. 
bioRxiv preprint doi: https://doi.ora/10.1101/704957: this version posted Julv 16. 2019. The copvriaht holder for this preprint (which was not certified by peer review) is the author/funder, who has granted bioRxiv a license to display the preprint in perpetuity. It is made available under aCC-BY-NC-ND 4.0 International license.

Figure 5
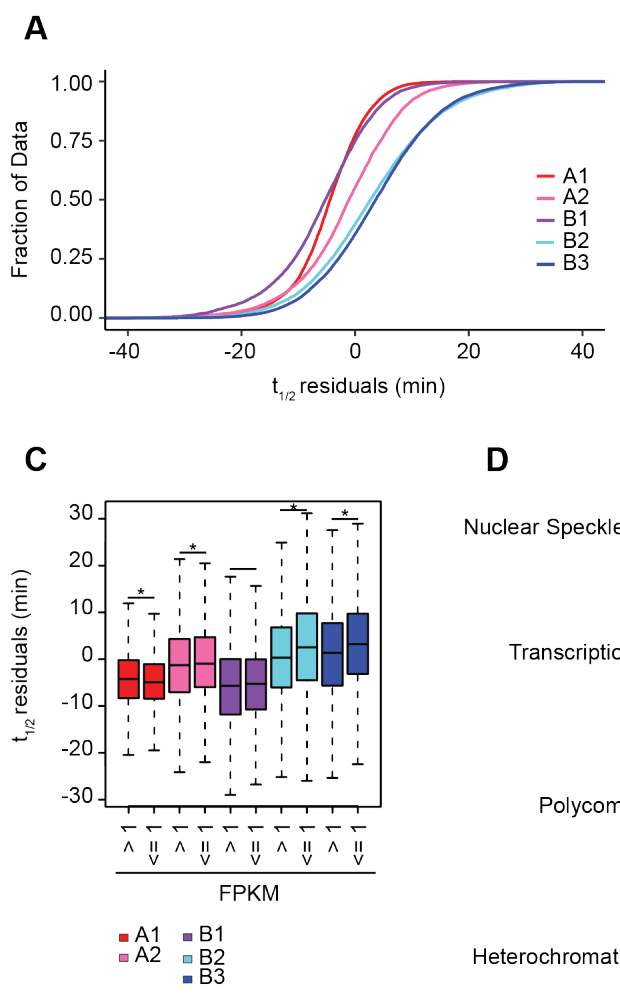

D
Nuclear Speckles

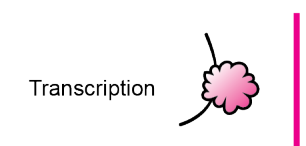

B

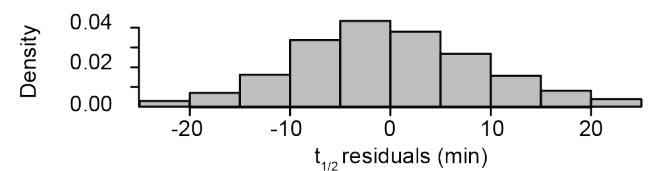

Repli-seq G1 Repli-seq S1

Repli-seq S2

Repli-seq S3

Repli-seq 54

Repli-seq G2

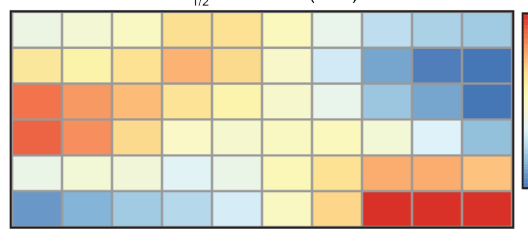

$+0.6$

$0.0 \stackrel{0}{0}$

$-0.6$

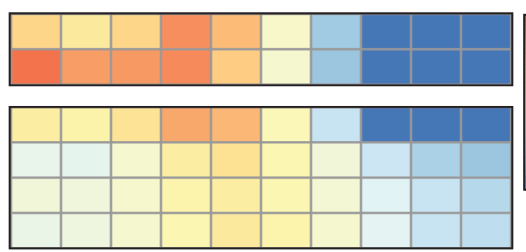

$+0.6$

pSC35

Pol II

H3K9ac

$\mathrm{H} 3 \mathrm{~K} 27 \mathrm{ac}$

H3K4me3
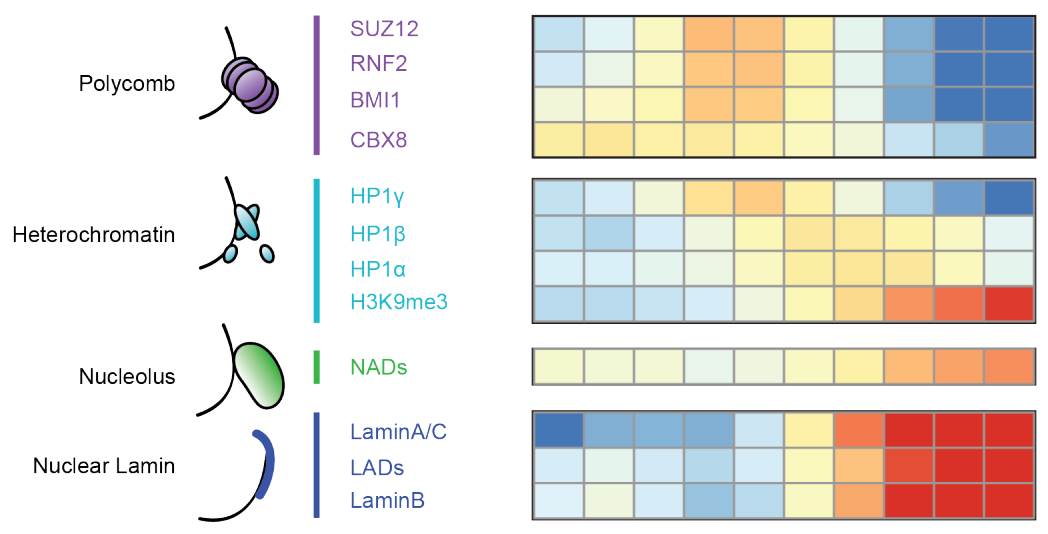
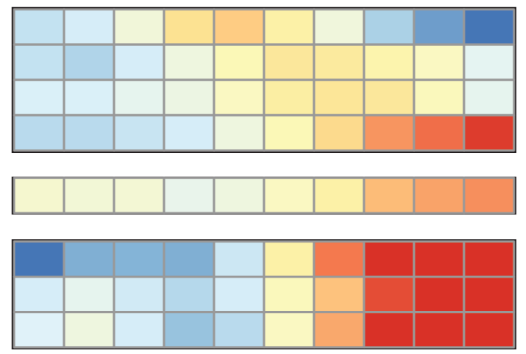

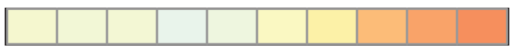

E

Predigest - Dpnll
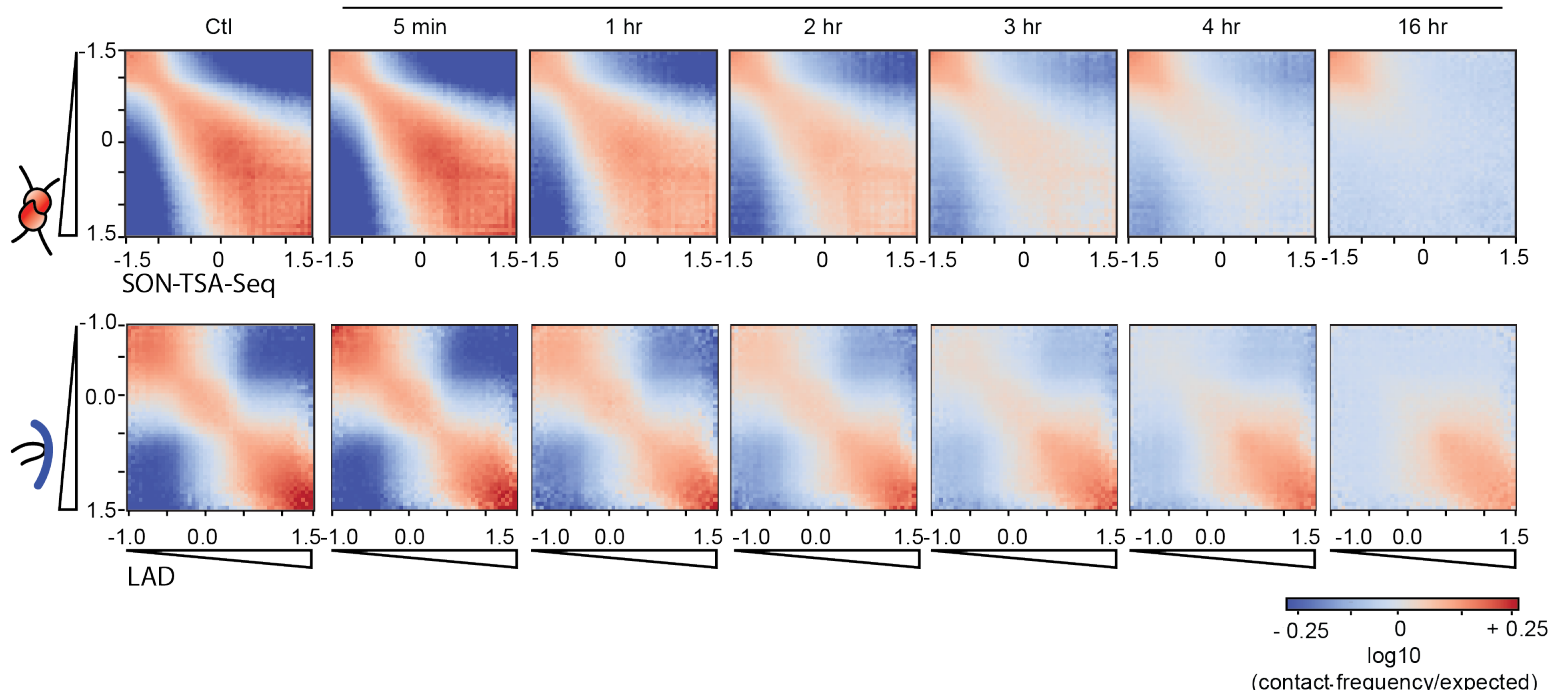

(contact-frequency/expected) 
Figure 5: Dissociation kinetics of chromatin interactions at different sub-nuclear structures

(A) Cumulative distributions of residuals of $\mathrm{t}_{1 / 2}$ (in minutes) for each of the five annotated subcompartments.

(B) Top: the genome was split into 10 bins, where each bin corresponds to sets of loci that share the same $t_{1 / 2}$ residual interval. Bottom: For each $t_{1 / 2}$ residual interval the mean z-score signal of Repli-Seq data in different phases of the cell cycle G1, S1-4, G2 .

(C) Boxplot of $t_{1 / 2}$ residuals for bins with expressed genes (mean FPKM $>1$ ) and bins with low or no expression (mean FPKM <=1) stratified by sub-compartment. Significance determined by two-sample two tailed $t$-test ${ }^{*}(p<0.003)$.

(D) Heatmap of mean z-score signal enrichment for various markers of sub-nuclear structures (See Methods) stratified by $t_{1 / 2}$ residual intervals (top of panel $B$ ). For loci in each $t_{1 / 2}$ residual interval the mean z-score was quantified for different chromatin features.

(E) Homotypic interaction saddle plots for loci ranked by their association with speckles (as detected by SON-TSA-seq, top (Chen et al., 2018)) and by their association with the nuclear lamina. Preferential pairwise interactions between loci associated with the lamina can still be observed after several hours, whereas preferential pair-wise interactions between loci associated with speckles are lost more quickly.

The strong correlation between active chromatin and relatively unstable chromatin interactions led us to examine the role of transcription in more detail (Figure $5 \mathrm{C}$ ). For each subcompartment, we split loci into expressed (FPKM>=1) or not expressed $(F P K M<1)$ categories. We find that sub-compartment status is the major determinant of chromatin interaction stability, irrespective of transcriptional status. However, transcriptional status modulates $t_{1 / 2}$ to some extent: in general, loci located in B2 and B3 sub-compartments are engaged in relatively stable chromatin interactions, but interactions that involve loci that are expressed have shorter halflives. Conversely, the expression status of loci located in the A1, A2 sub-compartments had only very minor effect on the residual $t_{1 / 2}$. Expressed loci in A1 or $A 2$ sub-compartments had slightly longer and shorter residual $t_{1 / 2}$ values. Although statistically significant, the average difference was very small ( $\mathrm{A} 1$ difference $=0.87$ minutes; $\mathrm{A} 2$ difference $=0.68$ minutes) .

To explore the relationship between chromatin interaction stability, chromatin state, and association at and around sub-nuclear structures in more detail, we leveraged the wealth of chromatin state data available for K562 cells (Figure 5D, Figure S6A, B). Chen et al. mapped loci in K562 cells that are localized near nuclear speckles using the recently developed TSA-seq 
method (Chen et al., 2018). We find that loci near the speckle-associated protein pSC35 are engaged in the most unstable interactions in the genome. A similar result was obtained for an independent TSA-seq dataset for the speckle associated protein SON. Similarly, transcriptionally active loci, identified by ChIP-seq for a range of histone modifications and factors associated with open chromatin such as H3K4me3 and RNA Polll, were also involved in relatively unstable chromatin interactions, though for some marks the residual $t_{1 / 2}$ values varied more widely.

Interestingly, interactions for loci bound by polycomb complexes (a subset of which are in the B1 sub-compartment) were as unstable as active and speckle associated loci (Figure 5D, S6B). This suggests that polycomb-bound domains, are held together by highly dynamic interactions. Interestingly, half-lives differed for loci bound by different polycomb subunits. Loci with the shortest $t_{1 / 2}$ values are enriched specifically for binding the CBX8 subunit. An example of a large polycomb-bound domain in K562 cells is the HoxD cluster. The cluster is around $100 \mathrm{~kb}$ in size and covered by the polycomb subunits Suz12, RNF2, CBX8 and BMI1 and the histone modification H3K27me3 (Figure S6G). The half-life of chromatin interactions for loci in the HoxD cluster is relatively short.

Silent and closed chromatin loci around the nucleolus or at the nuclear lamina were generally engaged in the most stable interactions (Figure 5D). Chromatin interaction stabilities for loci associated with the three distinct heterochromatin proteins 1 (HP1) differed: chromatin interactions for loci associated with HP1 $\gamma(\mathrm{CBX} 3)$ were relatively unstable while interactions for loci associated with HP1 $\beta$ (CBX1) or HP1 $\alpha$ (CBX5) were more stable. This variation is in agreement with the chromosomal locations and dynamics of these three HP1 proteins. HP1 $\gamma$ is associated with active chromatin and mobile, while HP1 $\alpha$ and HP1 $\beta$ are typically found in constitutive heterochromatin near (peri) centromeres and are much less mobile (Dialynas et al., 2007). Further indications that heterochromatic loci can display a range of chromatin interaction stabilities dependent upon their precise chromatin composition comes from the observation that stability is modulated by the ratio of HP1 $\alpha$ binding and lamin association: interactions for loci that display high levels of HP1 $\alpha$ binding but low levels of lamin association are not as stable as those for loci with lower levels of HP1 $\alpha$ binding and higher lamin association (Figure $\mathrm{S} 6 \mathrm{H}$ ).

The differential stability of pair-wise chromatin interactions at different sub-nuclear structures can be directly visualized and quantified by plotting interaction frequencies between pairs of 40 
$\mathrm{kb}$ loci arranged by their level of factor binding to obtain homotypic interaction saddle plots (Figure 5E). In these plots, pair-wise interactions between loci enriched in factor binding are shown in the lower right corner, and pair-wise interactions between loci not bound by the factor are shown in the upper left corner. For instance, we observe preferential interactions between pairs of loci near speckles, as determined by SON TSA-Seq (Chen et al., 2018). After chromatin fragmentation with Dpnll, we observe a progressive loss of preferential interactions between speckle associated loci, while preferential interactions between non-speckle associated loci can be observed even after 16 hours. Conversely, after chromatin fragmentation, we find that preferential interactions between lamin-associated loci remain detectable even at late time points, while preferential interactions between loci not at the lamina disappear relatively fast.

\section{Chromatin loops dissociate upon chromatin fragmentation}

Enriched point-to-point looping interactions are detected as "dots" in $\mathrm{Hi}-\mathrm{C}$ interaction maps. The majority of these represent interactions between pairs of convergent CTCF sites (Rao et al., 2014). We were interested in determining the fate of such loops after chromatin fragmentation. We aggregated $\mathrm{Hi}-\mathrm{C}$ data for purified nuclei at pairs of sites that had previously been shown to engage in looping interactions in K562 cells (Rao et al., 2014). We readily detected these loops in intact purified nuclei (Figure 6A). After fragmentation with HindIII for 4 hours, loops remained present and appeared to become slightly stronger. However, fragmenting chromatin with Dpnll resulted in loss of loops over time. Although loops appeared somewhat increased at $t=5$ minutes after Dpnll digestion, they were greatly reduced at $\mathrm{t}=1$ hour and at later timepoints.

Chromatin loops are thought to be formed by loop extrusion mediated by cohesin that is blocked at convergent CTCF sites. Therefore, we assessed whether CTCF and cohesin binding to chromatin is affected by chromatin fragmentation. We fractionated proteins in chromatin-bound and soluble fractions using the previously described chromatin binding assay ((Liang and Stillman, 1997), Methods). In intact nuclei, most of the CTCF and cohesin is associated with chromatin (Figure 6B, C). Digesting chromatin with Hindlll did not lead to dissociation of CTCF or cohesin. However, fragmenting chromatin with Dpnll led to dissociation of cohesin after 1 hour, while CTCF binding was only weakly affected. We conclude that DNA fragmentation to $<6$ $\mathrm{kb}$ fragments, but not to $10-25 \mathrm{~kb}$ fragments, leads to loss of cohesin binding and loss of looping interactions. These results are consistent with earlier observations that showed that in yeast stable chromatin binding by cohesin requires intact DNA (Ciosk et al., 2000). These data can be interpreted in the context of the model where cohesin rings encircle DNA (pseudo-) topologically 
(Srinivasan et al., 2018). This model of binding predicts that when DNA is fragmented, the cohesin ring can slide off nearby free ends. Our observation that cohesin binding and loops are disrupted when chromatin is fragmented to $<6 \mathrm{~kb}$ fragments suggest that loops are maintained by the encirclement of cohesin rings around the loop bases bound by CTCF.

Figure 6

A

Control

\begin{tabular}{lllll}
\multicolumn{9}{c}{ Predigest -Dpnll } \\
\hline $5 \mathrm{~m}$ & $1 \mathrm{~h}$ & $2 \mathrm{hs}$ & $3 \mathrm{hs}$ & $4 \mathrm{hs}$
\end{tabular}

$$
\begin{gathered}
\text { Control } \\
\text { Hindlll }
\end{gathered}
$$

$5 \mathrm{~m} \quad 1 \mathrm{~h} \quad 2 \mathrm{hs} \quad 3 \mathrm{hs} \quad 4 \mathrm{hs} \quad 4 \mathrm{hs}$
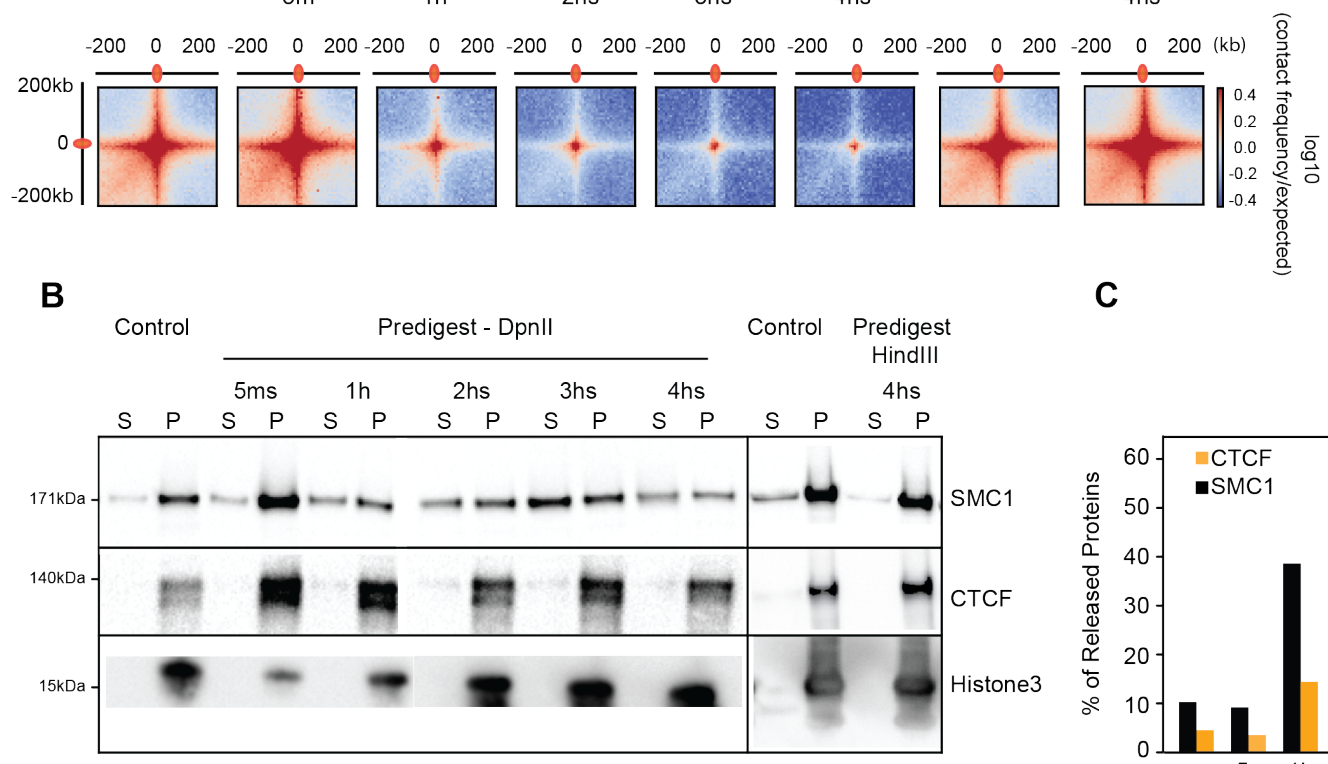

S: Supernatant

P: Pellet

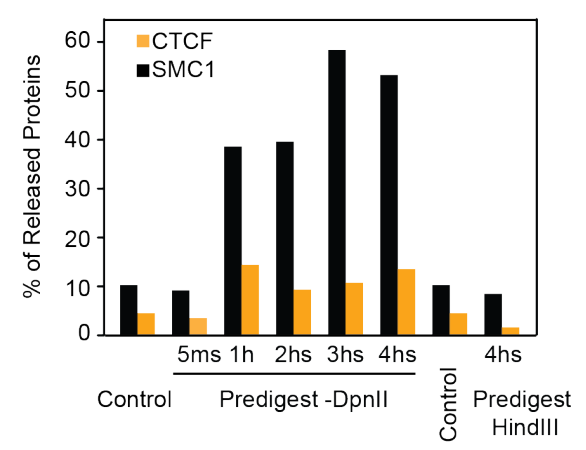

Figure 6: Chromatin loop dissociation upon fragmentation

(A) Aggregated distance-normalized $\mathrm{Hi}-\mathrm{C}$ interactions around 6,057 loops detected in $\mathrm{K} 562$ cells by HiCCUPS (Rao et al., 2014) at $10 \mathrm{~kb}$ resolution, for control nuclei and nuclei digested with Dpnll up to 4 hours, and for nuclei digested with HindllI for 4 hours.

(B) Western blot analysis of CTCF, cohesin and Histone H3 abundance in soluble and chromatin-bound fractions obtained from control nuclei and from nuclei pre-digested with Dpnll up to 4 hours and HindIII for 4 hours.

(C) Quantification of the data shown in panel B. Percentage of released protein is the ratio of protein level in the soluble fraction divided by the sum of the levels in the soluble and chromatin-bound fractions. 


\section{DISCUSSION}

$\mathrm{Hi}-\mathrm{C}$ interaction maps represent population-averaged folding of chromosomes but do not reveal whether individual pair-wise contacts are dynamic or stable. Liquid chromatin Hi-C reveals chromatin interaction stabilities genome-wide. In liquid chromatin $\mathrm{Hi}-\mathrm{C}$ chromatin is fragmented prior to fixation. After fragmentation, $\mathrm{Hi}-\mathrm{C}$ is then used at different time points to determine the extent to which the initially compartmentalized conformation of chromosomes is lost and the formerly spatially separated loci become mixed.

We observe an initial strengthening of $A / B$ compartmentalization following partial digestion. This result supports a "block copolymer" model of chromatin in the interphase nucleus, where B regions of chromosomes tend to cluster together, and A regions cluster together. Partial DNA digestion leads to a strengthening of compartmentalization by removing covalent linkages between $A$ and $B$ blocks, as long as the fragments are still large enough so that attractive forces between them are sufficient for phase segregation.

Further fragmentation of chromatin into pieces that are too short to maintain the phaseseparated state leads to progressive dissolution of chromatin conformation. The kinetics of this dissolution process provides insights into the attractive forces between chromatin segments, the intrinsic mobility of loci, the dynamics of nuclear organization, and the protein factors that can mediate chromatin interaction stability. Using liquid chromatin $\mathrm{Hi}-\mathrm{C}$ we obtain a view of the dynamics of chromatin interactions throughout the nucleus and the genome (Figure 7A). We find that chromatin dissolution is dependent on both chromatin state and fragmentation level. After correcting for fragmentation level we observe that chromatin interactions at different subnuclear structures differ in stability, with lamin-associated loci engaged in the most stable interactions and speckle and polycomb associated loci being most dynamic. Further, we find support for the model that CTCF-CTCF loops are stabilized by cohesin rings that encircle loop bases (Figure 7B).

Loci that are part of longer intact chromosomes display sub-diffusive dynamics, and their mobility is strongly constrained by the polymeric nature of chromatin and its folded state. In addition, their mobility is modulated by attractive interactions with other loci by factors that themselves are dynamic, and by local chromatin density. Live cell imaging experiments have examined locus motion extensively and found differences in mobility and constrained diffusion 
Figure 7

A

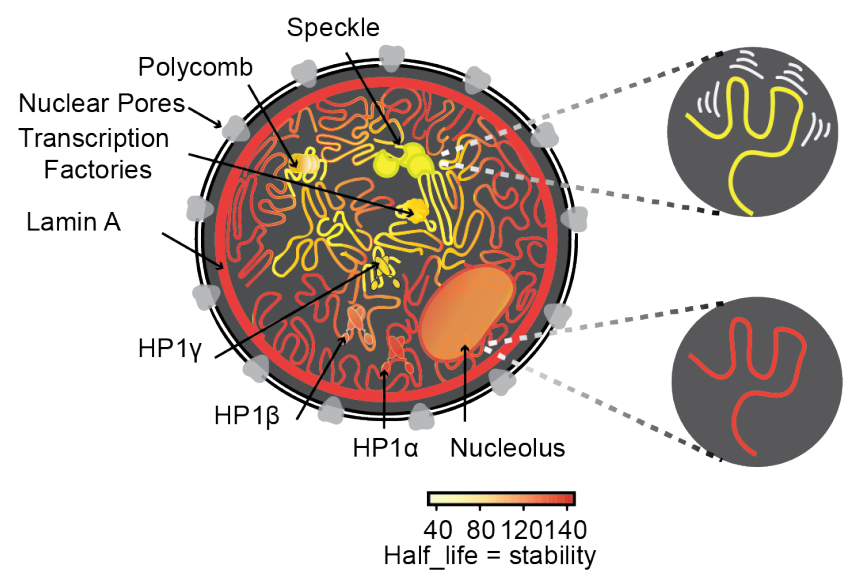

B

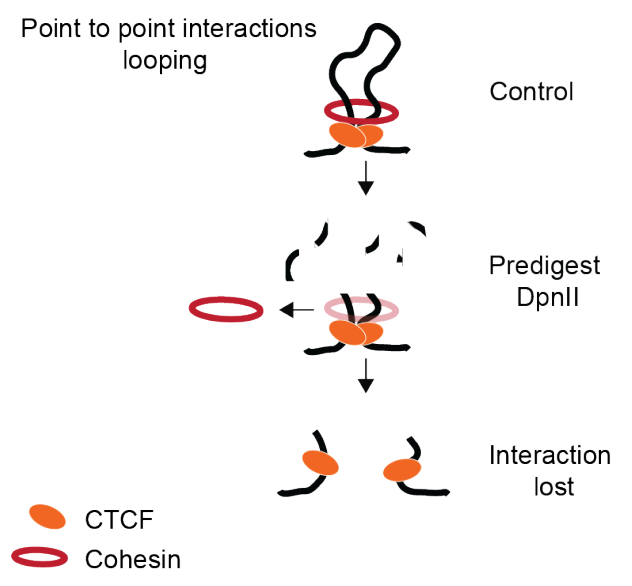

Figure 7: Illustration of chromatin interaction dynamics in the nucleus and model for cohesin loss after chromatin digestion

(A) Left: Schematic representation of varying chromatin interactions dynamics at different sub-nuclear domains. Shortest half-life reflects the least stable interactions (yellow), while longest half-life reflects the most stable interactions (dark orange). Nuclear subdomains differ greatly in their stability. Top right: Chromatin anchored at speckles is driven by the most dynamic interactions. Bottom right: Chromatin anchored at the nuclear lamina involves the most stable interactions.

(B) Model for how cohesin rings stabilize CTCF-CTCF loops by encircling loop bases. Top: Cohesin ring encircles loop bases at convergent CTCF sites. Middle: Pre-digestion with Dpnll cuts loop into chromatin fragments $<6 \mathrm{~kb}$, and the cohesin ring can slide off nearby ends. Bottom: CTCF remains bound to digested chromatin fragments but interactions between CTCF-bound sites are lost.

dependent on sub-nuclear position and chromatin state and activity (Bronshtein et al., 2009; Bronshtein et al., 2015; Hediger et al., 2002; Marshall et al., 1997; Nagashima et al., 2019; Shinkai et al., 2016; Thakar et al., 2006; Therizols et al., 2010). In such experiments the movement detected is strongly constrained by the fact that loci are part of very long chromosomes. A previous key study, which inspired the current work, aimed to identify the factors that determine intrinsic locus-locus interactions and locus mobility by explicitly removing the strong polymeric constraint due to linkage (Gartenberg et al., 2004). In that work, the mobility of an individual locus and its preference for association with sub-nuclear structures was measured by imaging after excising the locus from its normal genomic location so that it was 
freed from the polymeric constraint (Gartenberg et al., 2004). Specifically, a silent locus was excised from a yeast chromosome and its intrinsic preference for association with other silent loci and the nuclear periphery was assessed and found to depend on specific protein complexes bound to these loci that are involved in gene silencing. In our liquid chromatin Hi-C experiments, we digest DNA in situ and the polymeric constraint on movement is removed for all loci simultaneously, in effect performing a genome-wide variant of the experiments performed by Gartenberg et al. After fragmentation, dynamics of locus mixing is mostly determined by interactions of individual fragments with each other and with sub-nuclear structures mediated by local chromatin-associated factors, histone modifications, and chromatin density.

We show that chromosomal compartmentalization can tolerate genome-wide fragmentation with HindIII in >10-25 kb fragments. Our micro-mechanical elasticity measurements also show that chromosomes remain mechanically fully connected in those conditions. These results indicate that interactions between $10-25 \mathrm{~kb}$ fragments are stable enough to maintain the initially phaseor microphase- separated state of the nucleus, at least for several hours. This is in agreement with recent independent locus-specific experiments. First, previous studies had suggested that regions of several hundred kb were long enough to correctly position themselves in vivo according to their chromatin state in the corresponding compartment (van de Werken et al., 2017). Second, super-enhancers that typically range in size between 10 and $25 \mathrm{~kb}$ were found to associate both in cis and in trans, especially in the absence of cohesin (Rao et al., 2017), indicating that interactions between these 10-25 kb elements are stable enough to facilitate their clustering. Third, high-resolution Hi-C analysis in Drosophila indicates that domains $\sim 25 \mathrm{~kb}$ in size can phase-separate according to their chromatin status (Rowley et al., 2019; Rowley et al., 2017). Similarly, in Drosophila, polycomb-bound loci can cluster together at polycomb bodies when these loci are at least tens of kilobases long (Cheutin and Cavalli, 2012). Combining all these data with our liquid chromatin $\mathrm{Hi}-\mathrm{C}$ results, we conclude that chromatin phase segregation can occur when domains of a particular chromatin state are at least $10 \mathrm{~kb}$. This number is not far from an independent estimate of the block size required for phase separation based on polymer simulations of preferred interactions between HP1-bound loci (20 kb, (MacPherson et al., 2018)). Notably, digestion studies indicate that mitotic chromosomes are also constrained by stable chromatin interactions spaced by approximately 15 kb (Poirier and Marko, 2002).

Our results obtained with Dpnll digestion where the genome is fragmented in $<6 \mathrm{~kb}$ fragments show that these fragments are too short to maintain stable phase-segregated domains. We find 
that the stability of interactions between $<6 \mathrm{~kb}$ fragments, and the rate of mixing of initially segregated loci depends both on the level of Dpnll fragmentation and on their chromatin state and association with sub-nuclear structures: interactions at the nuclear lamina are relatively stable, those near nuclear speckles and polycomb complexes are highly unstable, while interactions for loci associated with different heterochromatin proteins and the nucleolus displayed a range of intermediate stabilities. The dynamics of associations between loci are therefore determined by chromatin-associated factors, and may also be determined directly by the biochemical properties of histone tail modifications. For instance, in a recent study, the Rosen lab found that chromatin fragments can form droplets in vitro and that the dynamics of chromatin fragments within these droplets are dependent upon both $\mathrm{H} 1$ binding and histone acetylation (Gibson et al., 2019): acetylation of histones resulted in more mobility of fragments and reduced droplet formation, while binding of $\mathrm{H} 1$ led to stable droplet with strongly reduced mobility of chromatin fragments. These results are consistent with the dynamics we observe here for active and inactive chromatin fragments within liquid chromatin.

Our results allow a crude estimate of the Flory-Huggins $\chi$ parameter for A/B segregation of chromatin in the nucleus. Given that HindIII and Dpnll cut chromatin into segments of approximately $15 \mathrm{~kb}$ and $6 \mathrm{~kb}$ respectively, a reasonable estimate of the minimum length of fragments necessary to drive $A / B$ segregation is $N^{*}=11 \pm 4 \mathrm{~kb}$. Given that these fragments are small compared to the $A / B$ compartmentalization scale of a few $\mathrm{Mb}$, the fragments will be essentially A- or B-type (euchromatin or heterochromatin) homopolymers. For homopolymers, the critical length needed for phase separation is $N^{*}=2 / \chi$ (de Gennes, 1979), indicating $\chi=0.18$ $\pm 0.05 / \mathrm{kb}(\chi=0.03 \pm 0.01 /$ nucleosome $)$. Given that $\chi$ is in $k_{B} T$ units $\left(1 k_{B} T=0.6 \mathrm{kcal} / \mathrm{mol}\right.$ at room temperature), its small value indicates that the effective demixing interaction between nucleosomes is weak, consistent with a liquid-like phase-separation picture for $A$ and $B$ compartments, where regulation of chromatin organization and compartmentalization is possible by relatively small changes in nucleosome interactions (e.g., via histone modifications). While extremely crude (e.g., we have used the $\chi$ estimate for a dense polymer melt rather than for a concentrated solution) our data indicate a weak value for $\chi$, and that the interactions that are driving compartmentalization are a small fraction of a $\mathrm{kcal} /(\mathrm{mol}$.nucleosome).

Generally, heterochromatic loci are engaged in the most stable interactions. This implies that these associations play a dominant role in spatial compartmentalization of the nucleus, consistent with predictions made by simulations (Falk et al., 2019). However, there is a range of 
stabilities correlating with different types of heterochromatin. Interactions between loci at the nuclear laminar are the most stable, suggesting that these loci are firmly tethered to the lamina meshwork. Imaging experiments showed that loci near the lamina can become embedded within the lamina meshwork, and that lamin proteins themselves are also stably localized within the lamina (Broers et al., 1999).

Loci associated with the three different HP1 proteins display different dissociation kinetics. Interestingly, these differences correlate with different dynamics and sub-nuclear locations of the HP1 proteins in the nucleus. HP1 $\gamma(\mathrm{CBX} 3)$ binds relatively transiently to euchromatin, which may explain the dynamic nature of chromatin interactions between loci bound by this protein (Dialynas et al., 2007). On the other hand, interactions between loci enriched in HP1 $\alpha$ (CBX5) are more stable, which likely is the result of more stable HP1 $\alpha$ binding to heterochromatin (Strom et al., 2017). Further, the clustering of heterochromatic loci bound by HP1 $\alpha$ may be related to condensate formation by HP1 $\alpha$ proteins at and around heterochromatic loci (Strom et al., 2017).

Polycomb bound regions of DNA are domains of facultative heterochromatin critical to proper vertebrate and invertebrate development. These regions spatially cluster together to form polycomb bodies visible in cell nuclei (Saurin et al., 1998). Polycomb bodies are shown to compact their associated chromatin as a potential mechanism for gene repression (Boettiger et al., 2016; Francis et al., 2004; Grau et al., 2011). Interestingly, liquid chromatin Hi-C showed differences in chromatin interaction stability between facultative heterochromatic domains marked by polycomb and constitutive heterochromatic domains marked by lamina association or binding of HP1 $\alpha / \mathrm{HP} 1 \beta$ proteins. While many chromatin contacts in constitutive heterochromatin were maintained even after 16 hours of digestion, the half-life for chromatin contacts at polycomb-bound regions was short, on a scale similar to more open and active regions of the genome. Thus, while polycomb domains and constitutive heterochromatin domains can both spatially segregate, when they are long enough, their biophysical nature is very different. The compacted states of polycomb and HP1 $\alpha$ bound chromatin appear to form via a similar phase-separation mechanism mediated by multivalent interactions between specific CBX homologs. In vitro and in vivo, both CBX2 (polycomb subunit) and CBX5 (HP1 1 ) are capable of forming condensates of polycomb bodies and constitutive heterochromatin, respectively (Larson et al., 2017; Plys et al., 2019; Tatavosian et al., 2019). Our data indicate that these different structures or condensates and associated chromatin have very different 
properties. Both are dynamic with regards to the exchange of proteins and both have a fraction of stably bound proteins (Strom et al., 2017; Youmans et al., 2018), but the stability of interactions between loci mediated by these factors is distinct, possibly related to differences in affinity between CBX proteins and chromatin: the binding affinity of CBX5 ( $\mathrm{Hp} 1 \alpha)$ for its preferential histone modification $\mathrm{H} 3 \mathrm{~K} 9 \mathrm{me} 3$ is much higher than the affinity of $\mathrm{CBX} 2$ for its preferential mark H3K27me3 (Kaustov et al., 2011).

Highly dynamic interactions occurred between loci associated with speckles. This is interesting because previous work had identified speckles to be one of two structural anchors, the other one being the lamina, that determine the organization of the nucleus (Chen et al., 2018; Quinodoz et al., 2018). Our data indicate that these structures differ greatly in how they anchor chromosome conformation: associations at the lamina involve stable interactions while anchoring at speckles is driven by more dynamic interactions.

The dynamics we observe for loci after chromatin fragmentation is likely related to the intrinsic dynamics of those loci while being part of full-length chromosomes. This is confirmed by the fact that active and inactive chromatin are more dynamic and mobile as assessed by both live cell imaging and liquid chromatin $\mathrm{Hi}-\mathrm{C}$. Our finding that interactions between active and open chromatin are more dynamic is consistent with observations that treating cells with histone deacetylase inhibitors, which leads to hyperacetylated chromatin, results in a reduced nuclear stiffness as detected by micromechanical measurements (Stephens et al., 2018). The reduced stiffness indicates weaker chromatin - chromatin interactions when chromatin is hyperacetylated.

It is important to point out that during the liquid chromatin $\mathrm{Hi}-\mathrm{C}$ procedure, some chromatin factors may dissociate from the nucleus, and this could affect the locus mixing behavior we observe. This will likely be the case for proteins that rapidly dissociate. As we discussed above, protein binding dynamics, e.g., of HP1 proteins, may determine the stability of chromatin interactions. An example of proteins that dissociate from chromatin upon fragmentation is the cohesin complex, possibly due to the way it binds chromatin by encircling chromatin fiber(s). However, the cohesin complex is not involved in compartment formation (Haarhuis et al., 2017; Nora et al., 2017; Nuebler et al., 2018; Rao et al., 2017; Schwarzer et al., 2017; Wutz et al., 2017), and thus its dissociation may not influence the stability of phase separation-driven chromatin interactions detected by liquid chromatin $\mathrm{Hi}-\mathrm{C}$. 
The current work analyzed the intrinsic chromatin interaction strengths and dissolution kinetics of chromosome conformation within otherwise inactive nuclei and how these measurements along chromosomes relate to chromatin state and chromosomal compartmentalization. Future work can focus on how these kinetic properties change in cells or nuclei, where active processes such as transcription, replication, chromatin compaction and condensation, and loop extrusion are also acting, and on determining the roles of RNAs, protein complexes, and histone modifications in modulating the attractive forces between loci and the dynamics of genome folding in general. 


\section{Acknowledgements}

J.D. and J.F.M. acknowledge support from the National Institutes of Health Common Fund 4D Nucleome Program (U54-DK107980). This work was also supported by a grant from the National Human Genome Research Institute (NHGRI) to J.D. (HG003143), another NHGRI grant to Z.W. (HG009446), and by grants from the National Cancer Institute (U54-CA193419) and from the National Institutes of Health (R01-GM105847 to J.F.M. and K99-GM123195 to A.D.S.). J.D. is an investigator of the Howard Hughes Medical Institute. We thank Leonid A. Mirny and Edward J. Banigan for discussions and Jian Ma for sharing K562 sub-compartment assignments.

\section{Author contributions}

J.D conceived the project. H.B. performed all 3C, 5C, Hi-C and liquid chromatin $\mathrm{Hi}-\mathrm{C}$ and chromatin fractionation experiments. DLL performed restriction digestion efficiency (Dpnll-seq) experiments. A.D.S. performed micromechanical studies and analyzed the data. T.B. and H.B. analyzed data. S.V. contributed analysis tools for liquid chromatin Hi-C analysis. J.F.M. provided polymer scaling ideas relevant to data interpretation. All authors contributed to writing the manuscript.

\section{Declaration of Interests}

The authors declare no competing interests.

\section{Data availability}

All data will be made publicly available upon publication via GEO and the 4D Nucleome Data Coordination Center. Submission of the all sequencing data to GEO is in progress and the accession number will be made available to the reviewers as soon as we have the number and secure accession key.

\section{Code availability}

All code for data processing and analysis, described in detail in the Methods, is available through the following GitHub accounts:

https://github.com/dekkerlab/5C-CBFb-SMMHC-Inhib

https://github.com/dekkerlab/cMapping

https://github.com/dekkerlab/cworld-dekker

https://github.com/tborrman/Dpnll-seq 
https://github.com/tborrman/digest-Hi-C

https://github.com/hms-dbmi/hic-data-analysis-bootcamp

https://github.com/mirnylab/cooltools/tree/master/cooltools

\section{REFERENCES}

Alipour, E., and Marko, J.F. (2012). Self-organization of domain structures by DNA-loopextruding enzymes. Nucleic Acids Res 40, 11202-11212.

Banigan, E.J., Stephens, A.D., and Marko, J.F. (2017). Mechanics and Buckling of Biopolymeric Shells and Cell Nuclei. Biophys J 113, 1654-1663.

Belaghzal, H., Dekker, J., and Gibcus, J.H. (2017). Hi-C 2.0: An optimized Hi-C procedure for high-resolution genome-wide mapping of chromosome conformation. Methods 123, 56-65.

Bickmore, W.A., and van Steensel, B. (2013). Genome architecture: domain organization of interphase chromosomes. Cell 152, 1270-1284.

Boettiger, A.N., Bintu, B., Moffitt, J.R., Wang, S., Beliveau, B.J., Fudenberg, G., Imakaev, M., Mirny, L.A., Wu, C.T., and Zhuang, X. (2016). Super-resolution imaging reveals distinct chromatin folding for different epigenetic states. Nature 529, 418-422.

Bonev, B., and Cavalli, G. (2016). Organization and function of the 3D genome. Nat Rev Genet $17,772$.

Branco, M.R., and Pombo, A. (2006). Intermingling of Chromosome Territories in Interphase Suggests Role in Translocations and Transcription-Dependent Associations. PLoS Biol 4, e138. Broers, J.L., Machiels, B.M., van Eys, G.J., Kuijpers, H.J., Manders, E.M., van Driel, R., and Ramaekers, F.C. (1999). Dynamics of the nuclear lamina as monitored by GFP-tagged A-type lamins. J Cell Sci 112 ( Pt 20), 3463-3475.

Bronshtein, I., Israel, Y., Kepten, E., Mai, S., Shav-Tal, Y., Barkai, E., and Garini, Y. (2009). Transient anomalous diffusion of telomeres in the nucleus of mammalian cells. Phys Rev Lett 103, 018102.

Bronshtein, I., Kepten, E., Kanter, I., Berezin, S., Lindner, M., Redwood, A.B., Mai, S., Gonzalo, S., Foisner, R., Shav-Tal, Y., et al. (2015). Loss of lamin A function increases chromatin dynamics in the nuclear interior. Nat Commun 6, 8044.

Chen, Y., Zhang, Y., Wang, Y., Zhang, L., Brinkman, E.K., Adam, S.A., Goldman, R., van Steensel, B., Ma, J., and Belmont, A.S. (2018). Mapping 3D genome organization relative to nuclear compartments using TSA-Seq as a cytological ruler. J Cell Biol 217, 4025-4048.

Cheutin, T., and Cavalli, G. (2012). Progressive polycomb assembly on H3K27me3

compartments generates polycomb bodies with developmentally regulated motion. PLoS Genet 8, e1002465.

Chien, R., Zeng, W., Kawauchi, S., Bender, M.A., Santos, R., Gregson, H.C., Schmiesing, J.A., Newkirk, D.A., Kong, X., Ball, A.R., Jr., et al. (2011). Cohesin mediates chromatin interactions that regulate mammalian beta-globin expression. J Biol Chem 286, 17870-17878.

Cho, W.K., Spille, J.H., Hecht, M., Lee, C., Li, C., Grube, V., and Cisse, II (2018). Mediator and RNA polymerase II clusters associate in transcription-dependent condensates. Science 361 , 412-415.

Chong, S., Dugast-Darzacq, C., Liu, Z., Dong, P., Dailey, G.M., Cattoglio, C., Heckert, A., Banala, S., Lavis, L., Darzacq, X., et al. (2018). Imaging dynamic and selective low-complexity domain interactions that control gene transcription. Science 361.

Ciosk, R., Shirayama, M., Shevchenko, A., Tanaka, T., Toth, A., Shevchenko, A., and Nasmyth, K. (2000). Cohesin's binding to chromosomes depends on a separate complex consisting of Scc2 and Scc4 proteins. Mol Cell 5, 243-254. 
Courchaine, E.M., Lu, A., and Neugebauer, K.M. (2016). Droplet organelles? EMBO J 35, 16031612.

Cremer, T., and Cremer, C. (2001). Chromosome territories, nuclear architecture and gene regulation in mammalian cells. Nat Rev Genet 2, 292-301.

de Gennes, P.-G. (1979). Scaling theory of polymer physics (Cornell University Press).

de Wit, E., Vos, E.S., Holwerda, S.J., Valdes-Quezada, C., Verstegen, M.J., Teunissen, H., Splinter, E., Wijchers, P.J., Krijger, P.H., and de Laat, W. (2015). CTCF Binding Polarity Determines Chromatin Looping. Mol Cell 60, 676-684.

Dekker, J., Belmont, A.S., Guttman, M., Leshyk, V.O., Lis, J.T., Lomvardas, S., Mirny, L.A., O'Shea, C.C., Park, P.J., Ren, B., et al. (2017). The 4D nucleome project. Nature 549, 219-226. Dekker, J., and Mirny, L.A. (2016). The 3D genome as moderator of chromosomal communication. Cell 164, 1110-1121.

Dekker, J., Rippe, K., Dekker, M., and Kleckner, N. (2002). Capturing Chromosome Conformation. Science 295, 1306-1311.

Di Pierro, M., Zhang, B., Aiden, E.L., Wolynes, P.G., and Onuchic, J.N. (2016). Transferable model for chromosome architecture. Proc Natl Acad Sci U S A 113, 12168-12173.

Dialynas, G.K., Terjung, S., Brown, J.P., Aucott, R.L., Baron-Luhr, B., Singh, P.B., and Georgatos, S.D. (2007). Plasticity of HP1 proteins in mammalian cells. J Cell Sci 120, 34153424.

Dixon, J.R., Selvaraj, S., Yue, F., Kim, A., Li, Y., Shen, Y., Hu, M., Liu, J.S., and Ren, B. (2012). Topological domains in mammalian genomes identified by analysis of chromatin interactions. Nature 485, 376-380.

Dostie, J., Richmond, T.A., Arnaout, R.A., Selzer, R.R., Lee, W.L., Honan, T.A., Rubio, E.H., Krumm, A., Lamb, J., Nusbaum, C., et al. (2006). Chromosome Conformation Capture Carbon Copy (5C): A Massively Parallel Solution for Mapping Interactions between Genomic Elements. Genome Res 16, 1299-1309.

ENCODE-Project-Consortium (2012). An integrated encyclopedia of DNA elements in the human genome. Nature 489, 57-74.

Erdel, F., and Rippe, K. (2018). Formation of Chromatin Subcompartments by Phase Separation. Biophys J 114, 2262-2270.

Fakan, S., and Puvion, E. (1980). The ultrastructural visualization of nucleolar and extranucleolar RNA synthesis and distribution. Int Rev Cytol 65, 255-299.

Falk, M., Feodorova, Y., Naumova, N., Imakaev, M., Lajoie, B.R., Leonhardt, H., Joffe, B., Dekker, J., Fudenberg, G., Solovei, I., et al. (2019). Heterochromatin drives organization of conventional and inverted nuclei. Nature In 570, 395-399.

Feric, M., Vaidya, N., Harmon, T.S., Mitrea, D.M., Zhu, L., Richardson, T.M., Kriwacki, R.W., Pappu, R.V., and Brangwynne, C.P. (2016). Coexisting Liquid Phases Underlie Nucleolar Subcompartments. Cell 165, 1686-1697.

Francis, N.J., Kingston, R.E., and Woodcock, C.L. (2004). Chromatin compaction by a polycomb group protein complex. Science 306, 1574-1577.

Fudenberg, G., Abdennur, N., Imakaev, M., Goloborodko, A., and Mirny, L.A. (2017). Emerging Evidence of Chromosome Folding by Loop Extrusion. Cold Spring Harb Symp Quant Biol 82, 45-55.

Fudenberg, G., Imakaev, M., Lu, C., Goloborodko, A., Abdennur, N., and Mirny, L.A. (2016). Formation of chromosomal domains by loop extrusion. Cell Rep 15, 2038-2049.

Gartenberg, M.R., Neumann, F.R., Laroche, T., Blaszczyk, M., and Gasser, S.M. (2004). Sirmediated repression can occur independently of chromosomal and subnuclear contexts. Cell $119,955-967$. 
Gibcus, J.H., Samejima, K., Goloborodko, A., Samejima, I., Naumova, N., Nuebler, J., Kanemaki, M.T., Xie, L., Paulson, J.R., Earnshaw, W.C., et al. (2018). A pathway for mitotic chromosome formation. Science 359.

Gibson, B.A., Doolittle, L.K., Jensen, L.E., Gamarra, N., Redding, S., and Rosen, M.K. (2019).

Organization and regulation of chromatin by liquid-liguid phase separation. BioRxiv doi:

https://doi.org/10.1101/523662.

Grau, D.J., Chapman, B.A., Garlick, J.D., Borowsky, M., Francis, N.J., and Kingston, R.E.

(2011). Compaction of chromatin by diverse Polycomb group proteins requires localized regions of high charge. Genes Dev 25, 2210-2221.

Guelen, L., Pagie, L., Brasset, E., Meuleman, W., Faza, M.B., Talhout, W., Eussen, B.H., de Klein, A., Wessels, L., de Laat, W., et al. (2008). Domain organization of human chromosomes revealed by mapping of nuclear lamina interactions. Nature 453, 948-951.

Guo, Y., Xu, Q., Canzio, D., Shou, J., Li, J., Gorkin, D.U., Jung, I., Wu, H., Zhai, Y., Tang, Y., et al. (2015). CRISPR Inversion of CTCF Sites Alters Genome Topology and Enhancer/Promoter Function. Cell 162, 900-910.

Haarhuis, J.H.I., van der Weide, R.H., Blomen, V.A., Yanez-Cuna, J.O., Amendola, M., van Ruiten, M.S., Krijger, P.H.L., Teunissen, H., Medema, R.H., van Steensel, B., et al. (2017). The Cohesin Release Factor WAPL Restricts Chromatin Loop Extension. Cell 169, 693-707 e614. Haering, C.H., Farcas, A.M., Arumugam, P., Metson, J., and Nasmyth, K. (2008). The cohesin ring concatenates sister DNA molecules. Nature 454, 297-301.

Hediger, F., Neumann, F.R., Van Houwe, G., Dubrana, K., and Gasser, S.M. (2002). Live imaging of telomeres: $y K u$ and Sir proteins define redundant telomere-anchoring pathways in yeast. Curr Biol 12, 2076-2089.

Hug, C.B., Grimaldi, A.G., Kruse, K., and Vaquerizas, J.M. (2017). Chromatin Architecture Emerges during Zygotic Genome Activation Independent of Transcription. Cell 169, 216-228 e219.

Iborra, F.J., Pombo, A., Jackson, D.A., and Cook, P.R. (1996). Active RNA polymerases are localized within discrete transcription "factories' in human nuclei. J Cell Sci 109, 1427-1436. Ivanov, D., and Nasmyth, K. (2005). A topological interaction between cohesin rings and a circular minichromosome. Cell 122, 849-860.

Jost, D., Carrivain, P., Cavalli, G., and Vaillant, C. (2014). Modeling epigenome folding: formation and dynamics of topologically associated chromatin domains. Nucleic Acids Res 42, 9553-9561.

Kaaij, L.J.T., van der Weide, R.H., Ketting, R.F., and de Wit, E. (2018). Systemic Loss and Gain of Chromatin Architecture throughout Zebrafish Development. Cell Rep 24, 1-10 e14.

Kagey, M.H., Newman, J.J., Bilodeau, S., Zhan, Y., Orlando, D.A., van Berkum, N.L., Ebmeier, C.C., Goossens, J., Rahl, P.B., Levine, S.S., et al. (2010). Mediator and cohesin connect gene expression and chromatin architecture. Nature 467, 430-435.

Kang, Y., Kim, Y.W., Kang, J., Yun, W.J., and Kim, A. (2017). Erythroid specific activator GATA1-dependent interactions between CTCF sites around the beta-globin locus. Biochim Biophys Acta Gene Regul Mech 1860, 416-426.

Kaustov, L., Ouyang, H., Amaya, M., Lemak, A., Nady, N., Duan, S., Wasney, G.A., Li, Z., Vedadi, M., Schapira, M., et al. (2011). Recognition and specificity determinants of the human cbx chromodomains. J Biol Chem 286, 521-529.

Larson, A.G., Elnatan, D., Keenen, M.M., Trnka, M.J., Johnston, J.B., Burlingame, A.L., Agard, D.A., Redding, S., and Narlikar, G.J. (2017). Liquid droplet formation by HP1alpha suggests a role for phase separation in heterochromatin. Nature 547, 236-240.

Leibler, L. (1980). Theory of microphase separation in block copolymers. Macromolecules 13, 1602-1617. 
Liang, C., and Stillman, B. (1997). Persistent initiation of DNA replication and chromatin-bound MCM proteins during the cell cycle in cdc6 mutants. Genes Dev 11, 3375-3386.

Lieberman-Aiden, E., van Berkum, N.L., Williams, L., Imakaev, M., Ragoczy, T., Telling, A., Amit, I., Lajoie, B.R., Sabo, P.J., Dorschner, M.O., et al. (2009). Comprehensive mapping of long-range interactions reveals folding principles of the human genome. Science 326, 289-293. Liu, L., Shi, G., Thirumalai, D., and Hyeon, C. (2018). Chain organization of human interphase chromosome determines the spatiotemporal dynamics of chromatin loci. PLoS Comput Biol 14, e1006617.

MacPherson, Q., Beltran, B., and Spakowitz, A.J. (2018). Bottom-up modeling of chromatin segregation due to epigenetic modifications. Proc Natl Acad Sci U S A 115, 12739-12744.

Marshall, W.F., Straight, A., Marko, J.F., Swedlow, J., Dernburg, A., Belmont, A., Murray, A.W., Agard, D.A., and Sedat, J.W. (1997). Interphase chromosomes undergo constrained diffusional motion in living cells. Curr Biol 7, 930-939.

Marzahn, M.R., Marada, S., Lee, J., Nourse, A., Kenrick, S., Zhao, H., Ben-Nissan, G., Kolaitis, R.M., Peters, J.L., Pounds, S., et al. (2016). Higher-order oligomerization promotes localization of SPOP to liquid nuclear speckles. EMBO J 35, 1254-1275.

Matsen, M.W., and Schick, M. (1994). Stable and unstable phases of a linear multiblcok copolymer melt. Macromolecules 27, 7157-7163.

Michieletto, D., Orlandini, E., and Marenduzzo, D. (2016). Polymer model with epigenetic recoloring reveals a pathway for the de novo establishment and 3D organization of chromatin domains. Phys Rev X 6, 041047.

Nagano, T., Lubling, Y., Stevens, T.J., Schoenfelder, S., Yaffe, E., Dean, W., Laue, E.D., Tanay, A., and Fraser, P. (2013). Single-cell Hi-C reveals cell-to-cell variability in chromosome structure. Nature 502, 59-64.

Nagano, T., Lubling, Y., Varnai, C., Dudley, C., Leung, W., Baran, Y., Mendelson Cohen, N., Wingett, S., Fraser, P., and Tanay, A. (2017). Cell-cycle dynamics of chromosomal organization at single-cell resolution. Nature 547, 61-67.

Nagashima, R., Hibino, K., Ashwin, S.S., Babokhov, M., Fujishiro, S., Imai, R., Nozaki, T., Tamura, S., Tani, T., Kimura, H., et al. (2019). Single nucleosome imaging reveals loose genome chromatin networks via active RNA polymerase II. J Cell Biol.

Nasmyth, K. (2001). Disseminating the genome: joining, resolving, and separating sister chromatids during mitosis and meiosis. Annu Rev Genet 35, 673-745.

Naumova, N., Imakaev, M., Fudenberg, G., Zhan, Y., Lajoie, B.R., Mirny, L.A., and Dekker, J. (2013). Organization of the mitotic chromosome. Science 342, 948-953. Nir, G., Farabella, I., Perez Estrada, C., Ebeling, C.G., Beliveau, B.J., Sasaki, H.M., Lee, S.D., Nguyen, S.C., McCole, R.B., Chattoraj, S., et al. (2018). Walking along chromosomes with super-resolution imaging, contact maps, and integrative modeling. PLoS Genet 14, e1007872. Nora, E.P., Goloborodko, A., Valton, A.L., Gibcus, J.H., Uebersohn, A., Abdennur, N., Dekker, J., Mirny, L.A., and Bruneau, B.G. (2017). Targeted Degradation of CTCF Decouples Local Insulation of Chromosome Domains from Genomic Compartmentalization. Cell 169, 930-944 e922.

Nora, E.P., Lajoie, B.R., Schulz, E.G., Giorgetti, L., Okamoto, I., Servant, N., Piolot, T., van Berkum, N.L., Meisig, J., Sedat, J., et al. (2012). Spatial partitioning of the regulatory landscape of the X-inactivation centre. Nature 485, 381-385.

Nuebler, J., Fudenberg, G., Imakaev, M., Abdennur, N., and Mirny, L.A. (2018). Chromatin organization by an interplay of loop extrusion and compartmental segregation. Proc Natl Acad Sci U S A 115, E6697-E6706.

Padeken, J., and Heun, P. (2014). Nucleolus and nuclear periphery: velcro for heterochromatin. Curr Opin Cell Biol 28, 54-60. 
Pirrotta, V., and Li, H.B. (2012). A view of nuclear Polycomb bodies. Curr Opin Genet Dev 22, 101-109.

Plys, A.J., Davis, C.P., Kim, J., Rizki, G., Keenen, M.M., Marr, S.K., and Kingston, R.E. (2019). Phase separation of Polycomb-repressive complex 1 is governed by a charged disordered region of CBX2. Genes Dev.

Poirier, M.G., and Marko, J.F. (2002). Mitotic chromosomes are chromatin networks without a mechanically contiguous protein scaffold. Proc Natl Acad Sci U S A 99, 15393-15397.

Pombo, A., Jackson, D.A., Hollinshead, M., Wang, Z., Roeder, R.G., and Cook, P.R. (1999).

Regional specialization in human nuclei: visualization of discrete sites of transcription by RNA polymerase III. EMBO J 18, 2241-2253.

Quinodoz, S.A., Ollikainen, N., Tabak, B., Palla, A., Schmidt, J.M., Detmar, E., Lai, M.M., Shishkin, A.A., Bhat, P., Takei, Y., et al. (2018). Higher-Order Inter-chromosomal Hubs Shape 3D Genome Organization in the Nucleus. Cell 174, 744-757 e724.

Ramani, V., Deng, X., Quu, R., Gunderson, K.L., Steemers, F.J., Disteche, C.M., Noble, W.S., Duan, Z., and Shendure, J. (2017). Massively multiplex single-cell Hi-C. Nat Methods 14, 263266.

Rao, S.S.P., Huang, S.C., Glenn St Hilaire, B., Engreitz, J.M., Perez, E.M., Kieffer-Kwon, K.R., Sanborn, A.L., Johnstone, S.E., Bascom, G.D., Bochkov, I.D., et al. (2017). Cohesin Loss Eliminates All Loop Domains. Cell 171, 305-320 e324.

Rao, S.S.P., Huntley, M.H., Durand, N.C., Stamenova, E.K., Bochkov, I.D., Robinson, J.T., Sanborn, A.L., Machol, I., Omer, A.D., Lander, E.S., et al. (2014). A 3D map of the human genome at kilobase resolution reveals principles of chromatin looping. Cell 159, 1665-1680. Riggs, A.D. (1990). DNA methylation and late replication probably aid cell memory, and type I DNA reeling could aid chromosome folding and enhancer function. Philos Trans $R$ Soc Lond $B$ Biol Sci 326, 285-297.

Rowley, M.J., Lyu, X., Rana, V., Ando-Kuri, M., Karns, R., Bosco, G., and Corces, V.G. (2019). Condensin II Counteracts Cohesin and RNA Polymerase II in the Establishment of 3D Chromatin Organization. Cell Rep 26, 2890-2903 e2893.

Rowley, M.J., Nichols, M.H., Lyu, X., Ando-Kuri, M., Rivera, I.S.M., Hermetz, K., Wang, P., Ruan, Y., and Corces, V.G. (2017). Evolutionarily Conserved Principles Predict 3D Chromatin Organization. Mol Cell 67, 837-852 e837.

Sanborn, A.L., Rao, S.S., Huang, S.C., Durand, N.C., Huntley, M.H., Jewett, A.I., Bochkov, I.D., Chinnappan, D., Cutkosky, A., Li, J., et al. (2015). Chromatin extrusion explains key features of loop and domain formation in wild-type and engineered genomes. Proc Natl Acad Sci U S A 112, E6456-6465.

Saurin, A.J., Shiels, C., Williamson, J., Satijn, D.P., Otte, A.P., Sheer, D., and Freemont, P.S. (1998). The human polycomb group complex associates with pericentromeric heterochromatin to form a novel nuclear domain. J Cell Biol 142, 887-898.

Sawyer, I.A., Sturgill, D., and Dundr, M. (2018). Membraneless nuclear organelles and the search for phases within phases. Wiley Interdiscip Rev RNA, e1514.

Schwarzer, W., Abdennur, N., Goloborodko, A., Pekowska, A., Fudenberg, G., Loe-Mie, Y., Fonseca, N.A., Huber, W., C, H.H., Mirny, L., et al. (2017). Two independent modes of chromatin organization revealed by cohesin removal. Nature 551, 51-56.

Shi, G., Liu, L., Hyeon, C., and Thirumalai, D. (2018). Interphase human chromosome exhibits out of equilibrium glassy dynamics. Nat Commun 9, 3161.

Shinkai, S., Nozaki, T., Maeshima, K., and Togashi, Y. (2016). Dynamic Nucleosome Movement Provides Structural Information of Topological Chromatin Domains in Living Human Cells. PLoS Comput Biol 12, e1005136.

Simonis, M., Klous, P., Splinter, E., Moshkin, Y., Willemsen, R., de Wit, E., van Steensel, B., and de Laat, W. (2006). Nuclear organization of active and inactive chromatin domains uncovered by chromosome conformation capture-on-chip (4C). Nat Genet 38, 1348-1354. 
Spector, D.L., and Lamond, A.I. (2011). Nuclear speckles. Cold Spring Harb Perspect Biol 3. Splinter, E., Heath, H., Kooren, J., Palstra, R.J., Klous, P., Grosveld, F., Galjart, N., and de Laat, W. (2006). CTCF mediates long-range chromatin looping and local histone modification in the beta-globin locus. Genes Dev 20, 2349-2354.

Srinivasan, M., Scheinost, J.C., Petela, N.J., Gligoris, T.G., Wissler, M., Ogushi, S., Collier, J.E., Voulgaris, M., Kurze, A., Chan, K.L., et al. (2018). The Cohesin Ring Uses Its Hinge to Organize DNA Using Non-topological as well as Topological Mechanisms. Cell 173, 1508-1519 e1518. Stephens, A.D., Banigan, E.J., Adam, S.A., Goldman, R.D., and Marko, J.F. (2017). Chromatin and lamin $A$ determine two different mechanical response regimes of the cell nucleus. Mol Biol Cell 28, 1984-1996.

Stephens, A.D., Liu, P.Z., Banigan, E.J., Almassalha, L.M., Backman, V., Adam, S.A., Goldman, R.D., and Marko, J.F. (2018). Chromatin histone modifications and rigidity affect nuclear morphology independent of lamins. Mol Biol Cell 29, 220-233.

Strom, A.R., Emelyanov, A.V., Mir, M., Fyodorov, D.V., Darzacq, X., and Karpen, G.H. (2017). Phase separation drives heterochromatin domain formation. Nature 547, 241-245.

Tang, Z., Luo, O.J., Li, X., Zheng, M., Zhu, J.J., Szalaj, P., Trzaskoma, P., Magalska, A., Wlodarczyk, J., Ruszczycki, B., et al. (2015). CTCF-Mediated Human 3D Genome Architecture Reveals Chromatin Topology for Transcription. Cell 163, 1611-1627.

Tatavosian, R., Kent, S., Brown, K., Yao, T., Duc, H.N., Huynh, T.N., Zhen, C.Y., Ma, B., Wang, H., and Ren, X. (2019). Nuclear condensates of the Polycomb protein chromobox 2 (CBX2) assemble through phase separation. J Biol Chem 294, 1451-1463.

Thakar, R., Gordon, G., and Csink, A.K. (2006). Dynamics and anchoring of heterochromatic loci during development. J Cell Sci 119, 4165-4175.

Therizols, P., Duong, T., Dujon, B., Zimmer, C., and Fabre, E. (2010). Chromosome arm length and nuclear constraints determine the dynamic relationship of yeast subtelomeres. Proc Natl Acad Sci U S A 107, 2025-2030.

Tolhuis, B., Palstra, R.J., Splinter, E., Grosveld, F., and de Laat, W. (2002). Looping and Interaction between Hypersensitive Sites in the Active beta-globin Locus. Mol Cell 10, 14531465.

van de Werken, H.J.G., Haan, J.C., Feodorova, Y., Bijos, D., Weuts, A., Theunis, K., Holwerda, S.J.B., Meuleman, W., Pagie, L., Thanisch, K., et al. (2017). Small chromosomal regions position themselves autonomously according to their chromatin class. Genome Res 27, 922933.

Vietri Rudan, M., Barrington, C., Henderson, S., Ernst, C., Odom, D.T., Tanay, A., and Hadjur, S. (2015). Comparative Hi-C reveals that CTCF underlies evolution of chromosomal domain architecture. Cell Rep 10, 1297-1309.

Wang, S., Su, J.H., Beliveau, B.J., Bintu, B., Moffitt, J.R., Wu, C.T., and Zhuang, X. (2016). Spatial organization of chromatin domains and compartments in single chromosomes. Science 353, 598-602.

Wutz, G., Varnai, C., Nagasaka, K., Cisneros, D.A., Stocsits, R.R., Tang, W., Schoenfelder, S., Jessberger, G., Muhar, M., Hossain, M.J., et al. (2017). Topologically associating domains and chromatin loops depend on cohesin and are regulated by CTCF, WAPL, and PDS5 proteins. EMBO J.

Xiong, K., and Ma, J. (2019). Revealing Hi-C subcompartments by imputing high-resolution inter-chromosomal interactions. BioRxiv doi: $h$ ttps://doi.org/10.1101/505503.

Youmans, D.T., Schmidt, J.C., and Cech, T.R. (2018). Live-cell imaging reveals the dynamics of PRC2 and recruitment to chromatin by SUZ12-associated subunits. Genes Dev 32, 794-805.

Zhu, L., and Brangwynne, C.P. (2015). Nuclear bodies: the emerging biophysics of nucleoplasmic phases. Curr Opin Cell Biol 34, 23-30. 
bioRxiv preprint doi: https://doi.org/10.1101/704957; this version posted July 16, 2019. The copyright holder for this preprint (which was not certified by peer review) is the author/funder, who has granted bioRxiv a license to display the preprint in perpetuity. It is made available under aCC-BY-NC-ND 4.0 International license.

Zidovska, A., Weitz, D.A., and Mitchison, T.J. (2013). Micron-scale coherence in interphase chromatin dynamics. Proc Natl Acad Sci U S A 110, 15555-15560. 


\section{METHODS}

\section{Digestion, cross-linking and copolymer architecture and hetero/euchromatin phase separation}

We have found that moderate digestion leads to formation of stronger inter-compartment interactions in $\mathrm{Hi}-\mathrm{C}$. While this may seem somewhat paradoxical at first glance, this effect is rather straightforward to explain if we remember two basic physical facts about chromatin in the G1 nucleus. First, individual chromosomes have a "blocky" structure, with hundreds of kilobasescale stretches of alternatingly heterochromatin-like and euchromatin-like character along their length. Second, the chromatin is in a state of quite extensive "crosslinking" (i.e., noncovalent chromatin-chromatin interactions mediated by proteins including gene regulatory factors such as cohesin, and heterochromatin linkers such as HP1 $\alpha$.

Therefore, chromatin in the G1 nucleus can be considered as a set of blocks of euchromatin and heterochromatin (the $A$ and $B$ compartments consisting of regions of predominantly euchromatin vs heterchromatin, respectively), which are constrained to be near each other by being part of the same linear chromosomes, i.e., effectively being long many-block copolymers. We suppose that the A and $B$ heterochromatin/

euchromatin monomers have a weak tendency to repel one another (or equivalently that A-A or B-B attract one another, for example via protein-mediated nucleosome-nucleosome interactions acting preferentially on euchromatin or heterochromatin, or even via physio-chemical effects such as relative hydrophobicity of more methylated nucleosomes).

If we suppose the $\mathrm{A}$ and $\mathrm{B}$ blocks to be on average $\mathrm{N}$ monomers long (roughly nucleosomes for the sake of this discussion), then under melt-like conditions the standard Flory theory of polymer phase separation predicts that if we were to cut the polymers into pure $A$ and $B$ blocks at the block boundaries (i.e., at a spacing of $\mathrm{N}$ monomers commensurate with the block sizes), they would phase separate for a segment-segment interaction strength stronger than chi* $=2 / \mathrm{N}$ (de Gennes, 1979). Note that this level of interaction (given approximately in k_B T units) is proportional to $1 / \mathrm{N}$ where $\mathrm{N}$ is crudely in nucleosome units; for 200 kilobase blocks, we have approximately $N=1000$, indicating that small fractions of a $k \_B \quad T$ in effective $A / B$ repulsion or $A$ A or B-B attraction is sufficient to drive strong euchromatin/heterochromatin phase separation (Marko and Siggia, 1997).

Now if we were to instead cut less frequently than this, say at every second block boundary, so as to arrive at a system of $A B$ linear diblock copolymers each of length $2 \mathrm{~N}$ ( $\mathrm{N}$ monomers of $A$ followed by $\mathrm{N}$ monomers of $\mathrm{B}$ ), the constraint that the $\mathrm{A}$ and $\mathrm{B}$ blocks be connected suppresses phase separation, increasing the critical interaction (all other factors held constant) to chi $^{*}=$ 5.3/N (Leibler, 1980). In this case bulk phase separation cannot occur, but instead local, or "microphase separation" occurs, with formation of micelle-like or layered phase-separated structures. Nevertheless, for chi $\gg>$ chi $^{*}$, strong segregation of the A and B monomers can still occur. 
If we were to not cut at all, but rather to suppose that the chromosomes are very long multiblock copolymers, with many blocks each of $\mathrm{N}$ monomers alternating between $\mathrm{A}$ and $\mathrm{B}$

("ABABABAB... multiblock copolymers"), the critical interaction strength will rise with increasing number of blocks, approaching the limit chi ${ }^{*}=7.5 / \mathrm{N}$ for many blocks (Matsen and Schick, 1994). Therefore, starting from this limit, the tendency for chromosome domains to phase separate will be enhanced by cutting the chromosomes up into successively smaller pieces: as chromatin cutting increases from no cutting, we expect to see intensification of $A / B$ compartment contrast in the Hi-C map.

Now, if we cut too frequently, when the cuts become spaced smaller than the block size (cut spacing $\mathrm{M}<\mathrm{N}$ monomers), we will have the situation that the critical interaction strength will become chi $^{*}=2 / M>2 / N$, i.e., the cuts are frequent enough to suppress phase separation by decreasing the amount of interaction enthalpy per polymer "molecule". Therefore we expect that overly frequent cutting will cause a reduction in A/B compartment $\mathrm{Hi}-\mathrm{C}$ map contrast, i.e., for some intermediate level of cutting similar to the sizes of the $A$ and $B$ blocks, one will see a maximum level of $A / B$ compartment contrast.

There is also likely an effect of "crosslinking" ("chromatin cross-bridging"), which provides an additional level of constraint suppressing phase separation, above the linear-multiblock architecture of chromosomes. For example, taking linear diblock copolymers (N A monomers followed by N B monomers) and circularizating them raises the critical interaction for microphase separation from $5.3 / \mathrm{N}$ to $8.9 / \mathrm{N}$, nearly a factor of 2 (Marko, 1993).

Similarly, if we start with A and B homopolymers each of length $N$, constraining them to have their ends at a flat surface, thus forcing them to mix at the surface, increases the critical interaction for phase separation from 2/N up to 4.5/N (Marko and Witten, 1991), with microphase separation again occurring in the constrained case. Releasing chromatin crosslinking/cross-bridging constraints (which also will occur for chromatin cutting) will in general also reduce the interaction strength needed to drive phase separation, increasing $A / B$ compartment contrast in Hi-C maps.

In conclusion, basic polymer phase separation theory predicts that gradually increasing the cleavage of chromatin will gradually increase the intensity of $A / B$ compartment contrast in Hi-C maps until the cuts are spaced by approximately one A or B block; further cutting will reduce the intensity of phase separation and A/B compartment contrast. Notably, the nature of the segregation can be expected to be "microphase segregation" rather than bulk phase separation, until the number of cuts is sufficient to liberate A or B "homopolymer" segments.

\section{K562 nuclei purification}

Three sucrose cushions were made before starting nuclei purification. $30 \mathrm{~mL}$ of $30 \%$ sucrose [10 mM PIPES pH 7.4, $10 \mathrm{mM} \mathrm{KCl,} 2 \mathrm{mM} \mathrm{MgCl}$, pH adjusted to 7.4 using $1 \mathrm{~N} \mathrm{KOH}, 30 \%$ sucrose, $1 \mathrm{mM}$ DTT (added prior to use), 1:100 protease inhibitor (Thermo Fisher 78438) (added prior to use)] was transferred to a $50 \mathrm{~mL}$ tube, then $5 \mathrm{~mL}$ of $10 \%$ sucrose [10 mM PIPES 
$\mathrm{pH} 7.4,10 \mathrm{mM} \mathrm{KCl}, 2 \mathrm{mM} \mathrm{MgCl} 2,10 \%$ Sucrose, $1 \mathrm{mM}$ DTT (added prior to use), 1:100 protease inhibitor (added prior to use)] was slowly loaded in top of $30 \%$ sucrose, and the tubes were incubated at $4^{\circ} \mathrm{C}$ until needed. $\mathrm{K} 562$ cell pellets (100 million cells) were lysed using the following nuclear isolation procedure. After the cells were spun, the pellets were washed twice with $10 \mathrm{~mL}$ HBSS, then pelleted after each wash at $300 \mathrm{rpm}$ for $10 \mathrm{~min}$ at $4^{\circ} \mathrm{C}$. Cell pellets were dissolved in $15 \mathrm{~mL}$ nuclear isolation buffer [10 mM PIPES PH 7.4, $10 \mathrm{mM} \mathrm{KCl,} 2 \mathrm{mM} \mathrm{MgCl} 2,1$ mM DTT (added prior to use), 1:100 protease inhibitor (added prior to use)], pH adjusted to 7.4 using $1 \mathrm{M} \mathrm{KOH}$ ]. Then, cells were lysed on ice in a $15 \mathrm{~mL}$ Dounce homogenizer with pestle $A$ (KIMBLE Kontes 885002-0015) by moving the pestle slowly up and down 20 times, followed by incubation on ice for $20 \mathrm{~min}$ and another 20 strokes. Next, each $5 \mathrm{~mL}$ of lysed extract was loaded slowly on top of a sucrose cushion prepared earlier. Then the tubes were spun for 15 $\min$ at $800 \mathrm{~g}$ at $4^{\circ} \mathrm{C}$. The supernatant was removed carefully for a good recovery of the nuclei pellet in the bottom of the tube. Nuclei pellets were resuspended in $1 \mathrm{~mL}$ of HBSS, then spun for $5 \mathrm{~min}$ at $5,000 \mathrm{~g}$ at $4^{\circ} \mathrm{C}$ using a benchtop refrigerated centrifuge. Then, the nuclei pellet was resuspended in $3 \mathrm{~mL} \mathrm{HBSS}$, and $1 \mu \mathrm{L}$ was taken to quantify the nuclei before the $3 \mathrm{~mL}$ was split over two microfuge tubes and spun for $5 \mathrm{~min}$ at $5,000 \mathrm{~g}$ at $4^{\circ} \mathrm{C}$ using a benchtop refrigerated centrifuge. Finally, the nuclei pellet was dissolved into an adequate total volume to obtain 1 million nuclei per $0.1 \mathrm{~mL}$ of Nuclei storage buffer (NSB) [10 mM PIPES pH 7.4, $10 \mathrm{mM} \mathrm{KCl,} 2$ $\mathrm{mM} \mathrm{MgCl}$, 50\% glycerol, $8.5 \%$ sucrose, $1 \mathrm{mM}$ DTT (added prior to use), 1:100 protease inhibitor (added prior to use)]. Each $0.5 \mathrm{~mL}$ of NSB containing 5 million nuclei was transferred to a microfuge tube and stored at $-80^{\circ} \mathrm{C}$.

\section{C (Chromosome Conformation Capture)}

$3 \mathrm{C}$ was performed as described in "From cells to chromatin: Capturing snapshots of genome organization with 5C technology" (Ferraiuolo et al., 2012).

Crosslinking: $1.25 \mathrm{~mL}$ of $37 \%$ formaldehyde was added to $40 \mathrm{~mL}$ of HBSS. 50 million cells or nuclei were washed twice using $20 \mathrm{~mL}$ of HBSS and then pelleted at $500 \mathrm{~g}$ for $10 \mathrm{~min}$. The pellet was resuspended in $5 \mathrm{~mL}$ HBSS and then added to $41.25 \mathrm{~mL}$ of HBSS and formaldehyde (final formaldehyde concentration was 1\%). The sample was incubated at RT for 10 min on a rocking platform. Afterward, to stop cross-linking $2.5 \mathrm{~mL}$ of $2.5 \mathrm{M}$ glycine was added and samples were incubated at RT for 5 min on a rotating platform. To pellet the crosslinked cells or nuclei the sample was centrifuged at $800 \mathrm{~g}$ for $10 \mathrm{~min}$ at $4^{\circ} \mathrm{C}$. After discarding the supernatant the pellet was washed twice using HBSS. Next, the pellet was either processed immediately as described below or was stored at $-80^{\circ} \mathrm{C}$ after flash freezing using liquid nitrogen.

Cell lysis: (This step was included when cells are used, but was skipped for $3 \mathrm{C}$ with purified nuclei). Cells were lysed by adding $2 \mathrm{~mL}$ of cold lysis buffer [10 mM Tris- $\mathrm{HCl}(\mathrm{pH}=8.0), 10 \mathrm{mM}$ $\mathrm{NaCl}, 0.2 \%$ Igepal CA-630 (NP40)] and $20 \mu \mathrm{L}$ of $100 x$ Protease inhibitors. The sample was incubated on ice for $15 \mathrm{~min}$ to let the cells swell. The cells were lysed on ice using the homogenizer with pestle A (KIMBLE Kontes 885300-0002) by moving the pestle slowly up and down 30 times and incubating on ice for 1 min followed by another 30 strokes. The sample was transferred to two $1.5 \mathrm{~mL}$ microcentrifuge tubes, spun at 5,000 $\mathrm{g}$ at RT for $5 \mathrm{~min}$ using a benchtop centrifuge. 
Digestion: each pellet was washed using $1 \mathrm{~mL}$ cold $1 \mathrm{X}$ NEBuffer 2.1, then spun at 5,000 $\mathrm{g}$ for 5 min at RT using a benchtop centrifuge, afterward each pellet was resuspended in $250 \mu \mathrm{L}$ of $1 \mathrm{X}$ NEB2.1 buffer, and the two pellets were pooled $(\sim 500 \mu \mathrm{L}) .50 \mu \mathrm{L}$ aliquots of the suspension were transferred to 10 new $1.5 \mathrm{~mL}$ microfuge tube and $292 \mu \mathrm{L}$ of $1 \times$ NEBuffer 2.1 was added to each tube. Next, $38 \mu \mathrm{L}$ of $1 \%$ SDS was added per tube and mixed well, the samples were incubated at $65^{\circ} \mathrm{C}$ for $10 \mathrm{~min}$, then placed on ice. $44 \mu \mathrm{L}$ of $10 \%$ Triton $\mathrm{X}-100$ was added to each tube to quench SDS. Finally, $400 \mathrm{U}$ of EcoRI (NEB R0101L) was added per tube and incubated at $37^{\circ} \mathrm{C}$ overnight on a thermocycler (with $900 \mathrm{rpm}$ for $30 \mathrm{sec}$ every $4 \mathrm{~min}$ ).

Ligation: $86 \mu \mathrm{L}$ of $10 \%$ SDS was added to the digested samples and the samples were then incubated at $65^{\circ} \mathrm{C}$ for 30 min for EcoRI inactivation after which the tubes were placed on ice. Each sample was then transferred to a $15 \mathrm{~mL}$ conical tube and $7.69 \mathrm{~mL}$ of ligation mix was added [820 $\mu \mathrm{L}$ 10\% Triton X-100, $820 \mu \mathrm{L}$ 10x ligation buffer (500 mM Tris-HCl pH7.5, $100 \mathrm{mM}$ $\mathrm{MgCl} 2,100 \mathrm{mM}$ DTT), $82 \mu \mathrm{L} 10 \mathrm{mg} / \mathrm{mL}$ BSA, $82 \mu \mathrm{L} 100 \mathrm{mM}$ ATP and $5.886 \mu \mathrm{L}$ ultrapure distilled water]. Finally, $10 \mathrm{U}$ of T4 ligase (Invitrogen 15224090) was added per tube before incubation at $16^{\circ} \mathrm{C}$ for $2 \mathrm{hr}$ on a thermocycler (with $900 \mathrm{rpm}$ for $30 \mathrm{sec}$ every $4 \mathrm{~min}$ ).

Reverse Crosslinking: $50 \mu \mathrm{L}$ of $10 \mathrm{mg} / \mathrm{mL}$ proteinase K (Fisher BP1750I-400) was added per tube, the sample was incubated at $65^{\circ} \mathrm{C}$ for $4 \mathrm{hr}$ followed by a second addition of $50 \mu \mathrm{L} 10$ $\mathrm{mg} / \mathrm{mL}$ Proteinase $\mathrm{K}$ and overnight incubation at $65^{\circ} \mathrm{C}$ on a thermocycler (with $900 \mathrm{rpm}$ for 30 sec every $4 \mathrm{~min})$.

DNA purification: Tubes were cooled at room temperature, at this stage each tube contains $8.21 \mathrm{~mL}$ final volume. The samples from every two tubes were combined to a $50 \mathrm{~mL}$ conical tube $(\sim 16,42 \mathrm{~mL})$ to have five tubes in total. DNA was extracted by adding an equal volume of $17 \mathrm{~mL}$ of saturated phenol pH8.0: chloroform (1:1) (Fisher BP1750I-400) and vortexing for 3 min. Then the mix was transferred to a $15 \mathrm{~mL}$ phase-lock tube (Quiagen 129073) followed by spinning tubes at $5,000 \mathrm{~g}$ for $10 \mathrm{~min}$. The upper phase was taken to a $50 \mathrm{~mL}$ tube to start the second extraction. We added an equal volume of $17 \mathrm{~mL}$ saturated phenol $\mathrm{pH}$ 8.0: chloroform (1:1), vortexing for $1 \mathrm{~min}$. Then the upper phase was transferred to a $15 \mathrm{~mL}$ phase-lock tube, and tubes were centrifuged at $5,000 \mathrm{~g}$ for $10 \mathrm{~min}$. We pooled all the upper phases from all 5 tubes $\sim 85 \mathrm{~mL}$ into a single $300 \mathrm{~mL}$ high-speed centrifuge tube to precipitate the DNA. $8.5 \mathrm{~mL}$ (1/10 volume) of $3 \mathrm{M}$ sodium acetate $\mathrm{pH} 5.2$ was added and brief vortexing was performed, then $212 \mathrm{~mL}$ (2.5 volumes) of ice-cold $100 \%$ ethanol was added, and the tube was inverted slowly several times and incubated at $-80^{\circ} \mathrm{C}$ for $1 \mathrm{hr}$. Afterward, the DNA was pelleted at 16,000 $\mathrm{g}$ for $30 \mathrm{~min}$ at $4^{\circ} \mathrm{C}$. The supernatant was discarded and the pellet was dissolved in $500 \mu \mathrm{L} 1 \mathrm{X}$ TLE and transferred to a $0.5 \mathrm{~mL}$ AMICON Ultra Centrifuge filter (UFC5030BK EMD Millipore). The column was centrifuged for $5 \mathrm{~min}$ at $14,000 \mathrm{~g}$ and the flow-through was discarded. The column was washed 4 times using $450 \mu \mathrm{L}$ of $1 X$ TLE for desalting DNA. After the final wash, the library remaining in the column $(\sim 50 \mu \mathrm{L})$ was eluted in $30 \mu \mathrm{L}$ of $1 \mathrm{XTLE}$, the column was flipped upside down into a new tube to collect DNA by centrifugation for 3 min at 4,000 g. RNA was degraded by adding $1 \mu \mathrm{L}$ of $10 \mathrm{mg} / \mathrm{mL}$ RNAase $A$ and incubation for $30 \mathrm{~min}$ at $37^{\circ} \mathrm{C}$.

Quality control assessment: to test the quality of the $3 \mathrm{C}$ library we used PCR to amplify a specific ligation product formed by two nearby restriction fragments, using the following primers: GPF33: GACCTCTGCACTAGGAATGGAAGGTTAGCC GPF23: GACTAATTCCTGACACTACTTGAGGGATAC 
The amplicon was digested with EcoRI to assess the efficiency of $3 \mathrm{C}$ ligation. $3 \mathrm{C}$ primers used for analysis of the beta-globin locus are listed in Supplemental Table S1.

\section{BAC library for 3C-PCR}

BAC DNA was generated as described (Dostie et al., 2006). A control ligation library covering the Beta-globin locus (ENCODE region ENm009) was generated using BACs overlapping the region. Starting with a mixture of DNA of seven BACs (CTC-775N13, RP11-715G8, CTD3048C22, CTD3055E11, CTD-2643I7, CTD-3234J1, and RP11-589G14) (Invitrogen), mixed in equimolar ratios, we used the same steps described in the $3 \mathrm{C}$ protocol above starting from the digestion step. BAC clones were digested with EcoRI, then randomly ligated, and the DNA was purified. The BAC ligation library reflects random ligation of EcoRI fragments throughout the beta-globin locus, so any difference in PCR signal for $3 \mathrm{C}$ primer pairs along the beta-globin locus due to differences in primer efficiency can be corrected by normalizing the amount of PCR product obtained with the $3 \mathrm{C}$ library to the amount obtained with the BAC ligation library.

\section{Chromosome Conformation Capture Carbon Copy (5C) \\ Experimental design}

Probes were designed as described (Dostie et al., 2006). $2135 \mathrm{C}$ probes were designed for a $\sim 1$ Mb region (chr11:4730996 -5729937; hg18) around the Beta-globin locus at EcoRI restriction sites using publicly available 5C primer design tools (Lajoie et al., 2009). Probes were designed according to a single alternating scheme exactly as described before (Lajoie et al., 2009) and the genomic uniqueness of all primers was verified with the SSAHA algorithm. For each EcoRI fragment at the $1 \mathrm{Mb}$ target region a primer was designed. $1045^{\prime}$ forward (FOR) and 109 5' reverse (REV) primers were designed. 5C primers are listed in Supplemental Table S2.

\section{Generation of $\mathbf{5 C}$ libraries}

$5 \mathrm{C}$ libraries were generated as described before (Ferraiuolo et al., 2012), with three modifications. First, we skipped the gel purification after the adaptor ligation and replaced this with a 1:1 Ampure step to remove unligated DNA and adaptors. Second, barcoded Illumina adaptors were used. Third, we performed the final PCR using TruSeq DNA LT kit Set A (REF 15041757).

Annealing: The $5 \mathrm{C}$ probes were pooled and combined with the $3 \mathrm{C}$ template each reaction contained 800,000 genome copies of $3 \mathrm{C}$ template and $0.2 \mathrm{fmol}$ per $5 \mathrm{C}$ probe $[800,000$ genome copies of 3C template, $2 \mu \mathrm{L}$ of 10X NEB4 (NEB B7004S), $2.75 \mu \mathrm{L}$ of Salmon Sperm DNA (250 ng; (Invitrogen ${ }^{\mathrm{TM}} 15632011$ ), $0.25 \mu \mathrm{L}$ of $1 \mathrm{fmol} / \mu \mathrm{L}$ probes , up to $20 \mu \mathrm{L}$ ultrapure distilled water]. We set up 8 annealing reactions for each library in a 96-well PCR plate. We then incubated the samples in a PCR machine and ran the following program $\left[95^{\circ} \mathrm{C}\right.$ for $9 \mathrm{~min}$, Ramp $0.1^{\circ} \mathrm{C} / \mathrm{sec}$ to $55 \mathrm{C}$, then keep at $55^{\circ} \mathrm{C}$ for $12 \mathrm{hr}$.

Ligation: We ligated $5 \mathrm{C}$ probe pairs, which represent a specific ligation junction in the $3 \mathrm{C}$ library, by adding $20 \mu \mathrm{L}$ of ligation mix [2 $\mu \mathrm{L}$ of [10X Taq DNA ligase buffer (NEB B0208S), 0.25 $\mu \mathrm{L}$ Taq DNA ligase (NEB M0208S), 17.75 uL ultrapure distiller water] while the samples are kept in the PCR block at $55^{\circ} \mathrm{C}$. We then incubated the reactions for $1 \mathrm{hr}$ at $55^{\circ} \mathrm{C}$ followed by a 
$10 \mathrm{~min}$ incubation at $65^{\circ} \mathrm{C}$; samples were then cooled to $4^{\circ} \mathrm{C}$. Negative controls (no ligase, no template, no $5 \mathrm{C}$ oligonucleotide) were included to ensure the absence of any contamination.

PCR amplification: Universal emulsion primers were used for amplification of the ligated product by using $5 \mathrm{C}$ forward and reverse emulsion primers [Forward_primer:

CCTCTCTATGGGCAGTCGGTGAT. Reverse_primer : CTGCCCCGGGTTCCTCATTCTCT] for $25 \mathrm{PCR}$ cycles [6 $\mu \mathrm{L}$ of ligation product, $2.5 \mu \mathrm{L}$ of 10XPCR $\left(600 \mathrm{mM}\right.$ Tris-SO $\mathrm{SO}_{4} \mathrm{pH} 8.9,180 \mathrm{mM}$ $\left.\left(\mathrm{NH}_{4}\right)_{2} \mathrm{SO}_{4}\right), 1.8 \mathrm{mM} \mathrm{MgCl} 2,0.2 \mathrm{mM}$ dNTP, $0.5 \mu \mathrm{L}$ F-emulsion primer $(80 \mu \mathrm{M}), 0.5 \mu \mathrm{L} \mathrm{R}-$ emulsion primer $(80 \mu \mathrm{M}), 0.225 \mu \mathrm{L}$ AmpliTaqÒ Gold DNA polymerase, ultrapure distilled water to bring volume up to $25 \mu \mathrm{L}$ ]. We then amplified DNA using this PCR program: [95 9 min, 25 cycles $\left.\left(95^{\circ} \mathrm{C} 30 \mathrm{~s}, 65^{\circ} \mathrm{C} 30 \mathrm{~s}, 72^{\circ} 30 \mathrm{~s}\right), 95^{\circ} \mathrm{C} 30 \mathrm{~s}, 65^{\circ} \mathrm{C} 30 \mathrm{~s}, 72^{\circ} \mathrm{C} 8 \mathrm{~min}, 4^{\circ} \mathrm{C}\right]$.

We pooled all the PCR reactions for the same library together and concentrated the DNA to 50 $\mu \mathrm{L}$ using $0.5 \mathrm{~mL}$ AMICON Ultra Centrifuge filter (UFC5030BK EMD Millipore). DNA was then loaded on a $2 \%$ agarose gel, along with a low molecular weight ladder, and the gel was run in a $4^{\circ} \mathrm{C}$ room at 200 volts for $90 \mathrm{~min}$. The $150 \mathrm{bp}$ DNA that corresponded to the ligated 5C probes was isolated from the gel using the QIAquick Gel Extraction Kit Protocol (QIAGEN 28115). DNA was finally eluted in $32 \mu \mathrm{L}$ of $1 \mathrm{XTLE}$.

A-tailing: A dATP was added to the 3 ' ends of the $5 \mathrm{C}$ library by adding $18 \mu \mathrm{L}$ of A-tailing mix [5 $\mu \mathrm{L}$ NEB buffer $2.1,10 \mu \mathrm{l}$ of $1 \mathrm{mM}$ dATP, $3 \mu \mathrm{L}$ Klenow exo (NEB M0212S)] to the $32 \mu \mathrm{L}$ of DNA sample from the previous step. The reaction was then incubated in a PCR machine $\left[a t 37^{\circ} \mathrm{C}\right.$ for $30 \mathrm{~min}$, then at $65^{\circ} \mathrm{C}$ for $20 \mathrm{~min}$, and finally cooled down to $4^{\circ} \mathrm{C}$ ]. Next, the tube was placed on ice immediately. 1:1 Ampure was used to remove unligated adaptors. The DNA was finally eluted in $40 \mu \mathrm{L} 1 \mathrm{X}$ T4 DNA Ligase buffer (Invitrogen).

Illumina adapter ligation and paired-end PCR: For this step, we used the TruSeq DNA LT kit Set A (REF 15041757). $10 \mu \mathrm{L}$ of ligation mix [5 $\mu \mathrm{L}$ Illumina paired-end adapters, $3 \mu \mathrm{L}$ T4 DNA ligase Invitrogen, $2 \mu \mathrm{L} 5 \mathrm{x}$ T4 DNA ligase buffer (Invitrogen 5X)] was added to the $40 \mu \mathrm{L}$ sample from the previous step. The ligation sample was then incubated at RT for 2 hours on a tube rotator. Afterward, the sample was run on a $2 \%$ agarose gel in a cold room $4^{\circ} \mathrm{C}$ at 150 volts for 120 min along with a low molecular weight ladder. The $270 \mathrm{bp}$ band that corresponds to $5 \mathrm{C}$ products (150 bp) ligated to the two adaptors (64 bp) was extracted from the gel and isolated using the QIAquick Gel Extraction Kit (QIAGEN 28115). DNA was finally eluted in $30 \mu \mathrm{L} 1 \mathrm{XTLE}$.

\section{Pre-digestion of nuclei (liquify chromatin)}

Purified nuclei as described above (K562 nuclei purification) were placed on ice and $1 \mathrm{~mL}$ of HBSS was added to each $0.5 \mathrm{~mL}$ of 5 million frozen nuclei. After thawing, nuclei were centrifuged $5 \mathrm{~min}$ at $5,000 \mathrm{~g}$. The nuclei pellet was washed twice with 1 XNEB3.1 for nuclei that would be digested with Dpnll or 1XNEB2.1 for nuclei that would be digested with HindIII. The nuclei were pelleted for $5 \mathrm{~min}$ at $5,000 \mathrm{~g}$ after each wash.

Isolated nuclei: a sample of 5 million nuclei was resuspended in 1,250 $\mu \mathrm{L}$ of $1 \mathrm{X}$ NEB3.1 as control, and then processed immediately for $\mathrm{Hi}-\mathrm{C}$ starting at the crosslinking step (see below $\mathrm{Hi}$ C 2.0 protocol).

Undigested nuclei: Each sample of two million nuclei was resuspended in $500 \mu \mathrm{L}$ of $1 \mathrm{X}$ NEB3.1 on ice, as and control for the pre-digestion and then treated as described immediately below. 
Dpnll pre-digestion: Each sample of two million nuclei was resuspended in $500 \mu \mathrm{L}$ of $1 \mathrm{X}$ NEB3.1 on ice. Next, $120 \mathrm{U}$ of Dpnll (NEB R0543S) was added to the sample in order to obtain

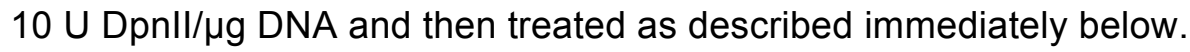

HindIII pre-digestion: Each sample of two million nuclei was resuspended in $500 \mu \mathrm{L}$ of $1 \mathrm{X}$ NEB2.1 on ice. Next, $600 \mathrm{U}$ of HindIII (NEB R0104T) was added to the sample in order to obtain $50 \mathrm{U}$ HindIII/ $\mu \mathrm{g}$ of DNA and then treated as described immediately below.

Next, control and pre-digestion samples were incubated at $37^{\circ} \mathrm{C}$ on a thermocycler $(900 \mathrm{rpm}$ for $30 \mathrm{sec}$ every $4 \mathrm{~min}$ ) for $5 \mathrm{~min}$ up to $16 \mathrm{~h}$. Afterward, samples were placed on ice for $10 \mathrm{~min}$.For Dpnll-seq and assessment of fragmentation level, a final volume of $10 \mathrm{mM}$ of EDTA was added to inactivate the endonuclease, followed immediately by the Dpnll-seq protocol (details of protocols below. Dpnll-Seq) or DNA purification for fragment analyzer analysis. For Hi-C, we proceeded immediately to the first step of the protocol (crosslinking as described below). For microscopy, nuclei samples were cross-linked with a $4 \%$ final concentration of paraformaldehyde.

\section{Hi-C 2.0}

$\mathrm{Hi}-\mathrm{C}$ was performed as described (Belaghzal et al., 2017) with some modifications in the crosslinking and lysis step as described below.

Crosslinking: isolated, undigested, and pre-digested (with liquified chromatin) nuclei were not pelleted after the pre-digestion step above but were crosslinked immediately as follows: for each sample $1,250 \mu \mathrm{L}$ volume of nuclei in the digestion buffer was transferred to a $21.875 \mathrm{~mL} \mathrm{mix}$ [ $625 \mu \mathrm{L}$ of $37 \%$ formaldehyde $+21.25 \mathrm{~mL}$ of HBSS]. For intact cells: 5 million K562 cells or nuclei were washed twice with $15 \mathrm{~mL}$ of HBSS and pelleted at $300 \mathrm{~g}$ for $10 \mathrm{~min}$, then resuspended in $2.5 \mathrm{~mL}$ of HBSS. The sample was transferred to $20.625 \mathrm{~mL}$ crosslinking mix [625 $\mu \mathrm{L}$ of $37 \%$ formaldehyde $+20 \mathrm{~mL}$ of HBSS].

All samples were incubated at RT for $10 \mathrm{~min}$ on a rocking platform. Next, to stop cross-linking $1.25 \mathrm{~mL}$ of $2.5 \mathrm{M}$ glycine was added to each sample and the mix was incubated at RT for $5 \mathrm{~min}$ on a rocking platform. To pellet the crosslinked cells/nuclei, the sample was centrifuged at 1,000 $\mathrm{g}$ for $10 \mathrm{~min}$ at $4^{\circ} \mathrm{C}$. The supernatant was discarded and the pellet was washed twice with HBSS before going to the next step or storing samples at $-80^{\circ} \mathrm{C}$.

Cells lysis: This step is not needed for isolated, undigested, and pre-digested (with liquified chromatin) nuclei. For $\mathrm{Hi}-\mathrm{C}$ with intact cells: the 5 million crosslinked cells were lysed by adding $1 \mathrm{~mL}$ cold lysis buffer [10 mM Tris- $\mathrm{HCl}(\mathrm{pH}=8.0), 10 \mathrm{mM} \mathrm{NaCl}, 0.2 \%$ Igepal CA-630 (NP40)] and $10 \mu \mathrm{L}$ of $100 \mathrm{X}$ Protease inhibitors. The sample was incubated on ice for $15 \mathrm{~min}$ to let the cells swell. The cells were lysed on ice using a dounce homogenizer with pestle A (KIMBLE Kontes 885300-0002) by moving the pestle slowly up and down 30 times and incubating on ice for 1 min followed by another 30 strokes. The sample was transferred to a new $1.5 \mathrm{~mL}$ microcentrifuge tube, and the sample was centrifuged at 5,000 $\mathrm{g}$ at RT for $5 \mathrm{~min}$.

Digestion: from each sample (isolated undigested, and pre-digested (with liquified chromatin) nuclei and lysed cells) the pellet was resuspended in $500 \mu \mathrm{L}$ of ice-cold $1 \mathrm{X}$ NEBuffer 3.1 , and pelleted for $5 \mathrm{~min}$ at $4,000 \mathrm{~g}$. The pellet was washed twice using $500 \mu \mathrm{L}$ of ice-cold $1 \mathrm{X}$ NEBuffer 3.1. After the last wash, the pellet was resuspended in $350 \mu \mathrm{L} 1 \mathrm{X}$ NEBuffer 3.1, and $8 \mu \mathrm{L}$ was taken and kept at $4^{\circ} \mathrm{C}$ to assess the DNA integrity later. $38 \mu \mathrm{L}$ of $1 \%$ SDS was added to $342 \mu \mathrm{L}$ $\left(380 \mu \mathrm{L}\right.$ total volume), and the mixture was resuspended and incubated for $10 \mathrm{~min}$ at $65^{\circ} \mathrm{C}$. The 
tube was placed on ice immediately afterward. Next $43 \mu \mathrm{L}$ of $10 \%$ Triton X-100 was added and the sample was mixed gently by pipetting. The tubes were placed at room temperature and 12 $\mu \mathrm{L}$ of $10 \mathrm{X}$ NEBuffer 3.1 was added. Then $400 \mathrm{U}$ of Dpnll (R0543L) was added and mixed gently before an overnight incubation at $37^{\circ} \mathrm{C}$ on a thermocycler (with $900 \mathrm{rpm}$ for 30 sec every $4 \mathrm{~min}$ ). Biotin Fill-in: After overnight digestion, the sample was incubated at $65^{\circ} \mathrm{C}$ for 20 min in order to inactivate the restriction enzyme. Then, $10 \mu \mathrm{L}$ of the digested sample was taken and kept at $4^{\circ} \mathrm{C}$ to assess the digestion efficiency later. DNA ends were marked with biotin-14-dATP by adding $60 \mu \mathrm{L}$ of biotin fill-in master mix [1XNEB 3.1, $0.25 \mathrm{mM}$ dCTP, $0.25 \mathrm{mM}$ dGTP, $0.25 \mathrm{mM}$ dTTP, $0.25 \mathrm{mM}$ biotin-dATP (ThermoFisher\#19524016), 50U Klenow polymerase Polymerase I (NEB M0210L)]. Next, the sample was incubated for $4 \mathrm{~h}$ at $23^{\circ} \mathrm{C}$ on a thermocycler (with $900 \mathrm{rpm}$ for $30 \mathrm{sec}$ every $4 \mathrm{~min}$ ). Finally, the sample was placed on ice immediately for 15 min before proceeding to the next step.

Ligation: After fill-in, the total sample volume was $\sim 535 \mu \mathrm{L}$. Ligation was performed by adding $665 \mu \mathrm{L}$ of ligation mix [240 $\mu \mathrm{L}$ of $5 x$ ligation buffer (1.8X) (Invitrogen), $120 \mu \mathrm{L} 10 \%$ Triton X-100, $12 \mu \mathrm{L}$ of $10 \mathrm{mg} / \mathrm{mL}$ BSA, $50 \mu \mathrm{L}$ T4 DNA ligase (Invitrogen 15224090), and $243 \mu \mathrm{L}$ ultrapure distilled water (Invitrogen)], to make a total volume of 1,200 $\mu \mathrm{L}$. The reaction was then incubated at $16^{\circ} \mathrm{C}$ for 4 hours in a Thermomixer with interval shake.

Reverse Crosslinking: $50 \mu \mathrm{L}$ of $10 \mathrm{mg} / \mathrm{mL}$ proteinase K (Fisher BP1750I-400) was added after ligation, the sample was incubated at $65^{\circ} \mathrm{C}$ for $4 \mathrm{hr}$ followed by a second addition of 50 $\mu \mathrm{L} 10 \mathrm{mg} / \mathrm{mL}$ Proteinase $\mathrm{K}$ and overnight incubation $65^{\circ} \mathrm{C}$

DNA purification: Reactions were cooled to room temperature and the $1.3 \mathrm{~mL}$ total volume was transferred to a $15 \mathrm{~mL}$ tube. The DNA was extracted by adding an equal volume of $1.3 \mathrm{~mL}$ of saturated phenol pH 8.0: chloroform (1:1) (Fisher BP1750l-400) and vortexing for $1 \mathrm{~min}$. Then the total volume of $2.6 \mathrm{~mL}$ was transferred to a $15 \mathrm{ml}$ phase-lock tube (Quiagen \#129065) and tubes were centrifuged at $5,000 \mathrm{~g}$ for $10 \mathrm{~min}$. The upper phase was transferred to a $15 \mathrm{~mL}$ tube to start the second extraction. An equal volume of $1.3 \mathrm{~mL}$ saturated phenol pH8.0: chloroform $(1: 1)$ was added and the sample was vortexed for $1 \mathrm{~min}$. Then the mix was transferred to $15 \mathrm{ml}$ phase-lock tube (Quiagen \#129065) followed by spinning tubes at 5,000 $\mathrm{g}$ for $10 \mathrm{~min}$. The upper phase of $\sim 1.3 \mathrm{~mL}$ was transferred to a $15 \mathrm{~mL}$ tube (high speed) to precipitate the DNA. 1/10 volume $(130 \mu \mathrm{L})$ of $3 \mathrm{M}$ sodium acetate $\mathrm{pH} 5.2$ was added and the sample was briefly vortexed. Then, 2.5 volumes of ice-cold $100 \%$ ethanol $3.25 \mathrm{~mL}$ was added, the tube was inverted slowly several times and then incubated at $-80^{\circ} \mathrm{C}$ for $1 \mathrm{hr}$. Next, the DNA was pelleted at $16,000 \mathrm{~g}$ for $30 \mathrm{~min}$ at $4^{\circ} \mathrm{C}$. The supernatant was discarded and the pellet was dissolved in $500 \mu \mathrm{L} 1 \mathrm{X}$ TLE and transferred to a $0.5 \mathrm{~mL}$ AMICON Ultra Centrifuge filter (UFC5030BK EMD Millipore). The column was spun for $5 \mathrm{~min}$ at $14,000 \mathrm{~g}$ and the flow-through was discarded. The column was washed 4 times using $450 \mu \mathrm{L}$ of $1 \mathrm{X}$ TLE for desalting of DNA. After the final wash the DNA remaining in the column $(\sim 50 \mu \mathrm{L})$ was eluted in $52 \mu \mathrm{L}$ of 1 XTLE. The column flipped upside down into a new tube to collect DNA and spun for $3 \mathrm{~min}$ at $4,000 \mathrm{~g}$, the volume was adjusted to $102 \mu \mathrm{L}$. RNA was degraded by adding $1 \mu \mathrm{L}$ of $10 \mathrm{mg} / \mathrm{mL}$ RNAase A and incubation for $30 \mathrm{~min}$ at $37^{\circ} \mathrm{C}$. To quantify the DNA concentration, $2 \mu \mathrm{L}$ of the final DNA sample along with the first 8 $\mu \mathrm{L}$ sample taken before digestion, the $10 \mu \mathrm{L}$ sample taken after digestion, and various amounts of the $1 \mathrm{~kb}$ ladder (NEB\#N3232s) were run on 1\% Agarose gel.

Removal of Biotin from unligated ends: To remove biotinylated nucleotides at DNA ends that did not ligate, the Hi-C sample was treated with T4 DNA polymerase. For each Hi-C sample, we 
assembled the following reaction: [up to $5 \mu \mathrm{g}$ of Hi-C library, $5 \mu \mathrm{L} \mathrm{10x} \mathrm{NEBuffer} \mathrm{3.1,0.025} \mathrm{mM}$ dATP, $0.025 \mathrm{mM}$ dGTP and $15 \mathrm{U}$ T4 DNA polymerase (NEB \# M0203L). The samples were brought up to $50 \mu \mathrm{L}$ total volume adding ultrapure distilled water. Reactions were incubated at $20^{\circ} \mathrm{C}$ for 4 hours, the enzyme was then inactivated by incubation of the reaction for 20 mins at $75^{\circ} \mathrm{C}$ and placed at $4^{\circ} \mathrm{C}$. Next, the samples were pooled and the volume was brought up to 130 $\mu L$ 1XTLE in preparation for sonication.

Sonication: the DNA was sheared to a size of 100-300 bp using a Covaris instrument [Duty cycle $10 \%$, Intensity 5, Cycles per Burst 200, set Mode Frequency sweeping, continuous degassing, process time $60 \mathrm{sec}$, Number of cycles] for 3 cycles. The volume was brought up to $500 \mu \mathrm{L}$ using TLE for Ampure fractionation.

Size fractionation using AMpure XP: $500 \mu \mathrm{L}$ AMpure beads (Beckman Coulter A63881) were added to a $1.5 \mathrm{~mL}$ tube labeled as $1.1 \mathrm{X}$. Then the tube was placed on the Magnetic Particle Separator (MPS) for $5 \mathrm{~min}$, and the supernatant was removed. Beads were resuspended in 150 $\mu \mathrm{L}$ AMpure mixture in order to make the $1.1 \mathrm{X}$ solution. $400 \mu \mathrm{L}$ of AMpure mixture was added to $500 \mu \mathrm{L}$ of sonicated DNA from the previous step and the tube was labeled $0.8 \mathrm{X}$. The sample was vortexed and spun down briefly followed by incubation at RT for 10 min on a rotating platform. Then the tube was placed on the MPS for 5 min at RT. The supernatants were collected and added to the 1.1X tube, the tube was briefly vortexed and spun down followed by incubation at RT for 10 min on a rotating platform. Then the tube was placed on the MPS for 5 min at RT. The supernatant was discarded and the beads in $0.8 \mathrm{X}$ and 1.1X tubes were washed twice with $1 \mathrm{~mL} \mathrm{70 \%} \mathrm{ethanol.} \mathrm{Beads} \mathrm{were} \mathrm{reclaimed} \mathrm{by} \mathrm{the} \mathrm{MPS} \mathrm{for} 5 \mathrm{~min}$. Beads were then airdried on the MPS until ethanol had evaporated completely. Next, $51 \mu \mathrm{L}$ of 1 XTLE was added to the $0.8 \mathrm{X}$ and 1.1X tubes to resuspend the DNA from the beads. Tubes were incubated at RT on a rotating platform for $10 \mathrm{~min}$. Then the tubes with AMpure beads from both $0.8 \mathrm{X}$ and $1.1 \mathrm{X}$ tubes were placed on the MPS for 5 min. Finally, the supernatants were transferred to $1.7 \mathrm{~mL}$ tubes labeled 0.8X and 1.1X. Our sample with DNA that ranges from 100-300 bp is in the 1.1X sample, the 0.8X sample was kept in case more DNA was needed. DNA from both samples $0.8 \mathrm{X}$ and $1.1 \mathrm{X}$ were quantified by running $1 \mu \mathrm{L}$ on a $2 \%$ agarose gel along with different amounts of low molecular weight DNA ladder (100 ng, 200 ng, 400 ng).

End Repair: $50 \mu \mathrm{L}$ of Hi-C sample was transferred to a PCR tube, then $20 \mu \mathrm{L}$ of the end-repair mix [3.5X NEB ligation buffer (NEB B0202S), $17.5 \mathrm{mM}$ dNTP mix, 7.5 U T4 DNA polymerase (NEBM0203L), 25 U T4 polynucleotide kinase (NEB M0201S), 2.5 U Klenow polymerase Polymerase I (NEB M0210L)] was added. The $70 \mu \mathrm{L}$ total volume reaction was then incubated at $37^{\circ} \mathrm{C}$ for $30 \mathrm{~min}$, followed by incubation at $65^{\circ} \mathrm{C}$ for $20 \mathrm{~min}$ to inactivate Klenow polymerase, and then the sample was put at $4^{\circ} \mathrm{C}$. The volume was brought up to $400 \mu \mathrm{L}$ using $1 \mathrm{X} \mathrm{TLE}$ for the next step.

Biotin pull-down: All the following steps were performed with $1.5 \mathrm{~mL}$ loBind tubes (Eppendorf 22431021). $15 \mu \mathrm{L}$ of MyOne streptavidin C1 beads mix (Thermo Fisher 65001) was transferred to a $1.5 \mathrm{~mL}$ tube. The beads were washed twice by adding $400 \mu \mathrm{L}$ of TWB [ $5 \mathrm{mM}$ Tris- $\mathrm{HCl}$ pH8.0, $0.5 \mathrm{mM}$ EDTA, $1 \mathrm{M} \mathrm{NaCl}, 0.05 \%$ Tween20] followed by incubation for $3 \mathrm{~min}$ at RT. The tube was then placed on an MPS for $1 \mathrm{~min}$ and the supernatant was removed. After the washes, the beads were resuspended in $400 \mu \mathrm{L}$ of $2 \mathrm{X}$ Binding Buffer (BB) [10 mM Tris- $\mathrm{HCl} \mathrm{pH} 8,1 \mathrm{mM}$ EDTA, $2 \mathrm{M} \mathrm{NaCl}$ ] and mixed with the $400 \mu \mathrm{L}$ DNA from the previous step in a new $1.5 \mathrm{~mL}$ tube. The mixture was incubated for $15 \mathrm{~min}$ at RT with rotation, the tube was then placed on the MPS 
for $1 \mathrm{~min}$ and the supernatant was removed. The DNA bound to the beads was washed by adding $400 \mu \mathrm{L}$ of $1 \mathrm{X} \mathrm{BB}$ and transferred to a new tube. The beads were reclaimed against the MPS for $1 \mathrm{~min}$, and the supernatant was discarded. The second wash used $100 \mu \mathrm{L}$ of $1 \mathrm{X}$ TLE, beads were reclaimed against MPS for $1 \mathrm{~min}$, and the supernatant was discarded. Finally, the DNA bound to the beads was eluted in $32 \mu \mathrm{L}$ of $1 \mathrm{X}$ TLE.

A-tailing: A dATP was added to the 3 ' ends by adding $18 \mu \mathrm{L}$ of A-tailing mix [ $5 \mu \mathrm{L}$ NEB buffer $3.1,10 \mu \mathrm{L}$ of $1 \mathrm{mM}$ dATP, $3 \mathrm{U}$ Klenow exo (NEB M0212S)] to the $32 \mu \mathrm{L}$ of DNA sample from the previous step. The reaction was incubated in a PCR machine [at $37^{\circ} \mathrm{C}$ for $30 \mathrm{~min}$, then at $65^{\circ} \mathrm{C}$ for $20 \mathrm{~min}$, followed by cool down to $4^{\circ} \mathrm{C}$ ]. Next, the tube was placed on ice immediately. The sample was transferred to a $1.5 \mathrm{~mL}$ loBind tube, the tube was placed on the MPS for $1 \mathrm{~min}$ and the supernatant was removed. The streptavidin beads bound to DNA were washed twice using $100 \mu \mathrm{L}$ 1X T4 DNA Ligase Buffer (Invitrogen). Finally, streptavidin beads bound to DNA were resuspended in $40 \mu \mathrm{L} 1 \mathrm{X}$ T4 DNA Ligase buffer (Invitrogen).

Illumina adapter ligation and paired-end PCR: For this step, the TruSeq DNA LT kit Set A (REF\#15041757) was used. $10 \mu \mathrm{L}$ of ligation mix [5 $\mu \mathrm{L}$ Illumina paired-end adapters, $3 \mu \mathrm{L}$ T4 DNA ligase Invitrogen, $2 \mu \mathrm{L} 5 x$ T4 DNA ligase buffer (Invitrogen 5X)] was added to the $40 \mu \mathrm{L} \mathrm{Hi-}$ C sample from the previous step. The ligation sample was then incubated at RT for 2 hours on a rotator. The sample was transferred to a $1.5 \mathrm{~mL}$ loBind tube, the tube was placed on the MPS for $1 \mathrm{~min}$ and the supernatant was removed. The streptavidin beads bound to DNA were washed twice with $400 \mu \mathrm{l}$ of TWB, then twice using $100 \mu \mathrm{L} 1 \mathrm{X}$ TLE. Finally, the sample was resuspended in $20 \mu \mathrm{L}$ Of 1 XTLE.

Illumina Truseq Kit for PCR: We performed three trial PCR reactions as follows [2.5 $\mu L$ DNA bound to beads, $2 \mu \mathrm{L}$ of Primers mix (TruSeq DNA LT kit Set A 15041757)), $10 \mu \mathrm{L}$ Master Mix (TruSeq DNA LT kit Set A 15041757), $10.5 \mu \mathrm{L}$ of ultrapure distilled water (Invitrogen)]. We split the $25 \mu \mathrm{L}$ over three PCR tubes $(5 \mu \mathrm{L}, 5 \mu \mathrm{L}, 15 \mu \mathrm{L}$ per tube). Each of the three samples was then amplified with different numbers of PCR cycles $(6,8,10$ respectively) to assess the Hi-C library quality: [ $30 \mathrm{sec}$ at $98^{\circ} \mathrm{C}, \mathrm{n}$ cycles of $\left(30 \mathrm{sec}\right.$ at $98^{\circ} \mathrm{C}, 30 \mathrm{sec}$ at $65^{\circ} \mathrm{C}, 30 \mathrm{sec}$ at $\left.72^{\circ} \mathrm{C}\right), 5$ min at $72^{\circ} \mathrm{C}$, hold at $10^{\circ} \mathrm{C}$ ]. $10 \mu \mathrm{L}$ was taken from the $15 \mu \mathrm{L}$ sample (with 10 PCR cycles), the $10 \mu \mathrm{L}$ sample was then digested with Clal for $1 \mathrm{~h}$ by adding $10 \mu \mathrm{L}$ of digestion mix [1.5 $\mu \mathrm{L} 10 \mathrm{x}$ NEB Cutsmart buffer, $1.5 \mu \mathrm{L}$ Clal ( NEB R0197S ), $7 \mu \mathrm{L}$ ultrapure distilled water]. The $5 \mu \mathrm{L}$ of each PCR cycle sample along with the $20 \mu \mathrm{L}$ digested sample, and titration of the low molecular ladder (100 ng, 200 ng, 400 ng) (NEB) were run on a 2\% Agarose gel. After digestion with Clal, a downward shift of the amplified DNA to smaller sizes is expected, which indicates DNA ends were correctly filled in and ligated (creating a Clal site). The number of PCR cycles to generate the final $\mathrm{Hi}-\mathrm{C}$ material for deep sequencing was chosen based the minimum number of PCR cycles in the PCR titration that was needed to obtain sufficient amounts of DNA for sequencing using the remaining $17.5 \mu \mathrm{L} \mathrm{Hi-C} \mathrm{sample.}$

\section{Dpnll-Seq}

For each Dpnll-Seq library, 10 million nuclei were used right after the pre-digestion procedure described above (Pre-digestion of nuclei). The pre-digested nuclei were then treated as follows: 
Proteinase K: $50 \mu \mathrm{L}$ of $10 \mathrm{mg} / \mathrm{mL}$ proteinase $\mathrm{K}$ (ThermoFisher \# 25530) was added to each $500 \mu \mathrm{L}$ pre-digested nuclei sample (2 million nuclei) (See Methods: Pre-digestion) and the 5 tubes were incubated at $65^{\circ} \mathrm{C}$ for 3 hours.

DNA purification: Tubes were cooled to room temperature and all 5 samples were pooled in a single $15 \mathrm{~mL}$ tube $(2.75 \mathrm{~mL}$ total volume). The DNA was extracted by adding an equal volume of $2.75 \mathrm{~mL}$ of saturated phenol pH8.0: chloroform (1:1) (Fisher BP1750I-400), followed by vortexing for $1 \mathrm{~min}$. The sample $(5.5 \mathrm{~mL})$ was transferred to a $15 \mathrm{~mL}$ phase-lock tube (Quiagen \#129065) followed by centriguation at $5,000 \mathrm{~g}$ for $10 \mathrm{~min}$. The upper phase was transferred to a $15 \mathrm{~mL}$ tube to start the second extraction. An equal volume of $2.75 \mathrm{~mL}$ saturated phenol $\mathrm{pH} 8.0$ : chloroform (1:1) was added, followed by vortexing for $1 \mathrm{~min}$. Then the mix was transferred to a $15 \mathrm{~mL}$ phase-lock tube (Quiagen \#129065) followed by centrifugation at 5,000 $\mathrm{g}$ for $10 \mathrm{~min}$. The upper phase of $\sim 2.75 \mathrm{~mL}$ was transferred to a $15 \mathrm{~mL}$ tube (high speed), 1/10 volume $(275 \mu \mathrm{L}$ ) $3 \mathrm{M}$ sodium acetate $\mathrm{pH} 5.2$ was added followed by brief vortexing and then 2.5 volumes of icecold $100 \%$ ethanol $(6.875 \mathrm{~mL})$ were added. The tube was inverted slowly several times, incubated at $-80^{\circ} \mathrm{C}$ for $1 \mathrm{hr}$ and then DNA was pelleted by centrifugation at $16,000 \mathrm{~g}$ for $30 \mathrm{~min}$ at $4^{\circ} \mathrm{C}$. The supernatant was discarded and the pellet was dissolved in $500 \mu \mathrm{L} 1 \mathrm{X}$ NEB3.1 and transferred to a $0.5 \mathrm{~mL}$ AMICON Ultra Centrifuge filter (UFC5030BK EMD Millipore). The column was centrifuged for $5 \mathrm{~min}$ at $14,000 \mathrm{~g}$ and the flow-through was discarded. The column was washed 4 times using $450 \mu \mathrm{L}$ of $1 \mathrm{X}$ NEB3.1 for desalting of DNA. After the final wash, the library remaining in the column $(\sim 50 \mu \mathrm{L})$ was eluted in $450 \mu \mathrm{L}$ of 1 XNEB3.1; the column was flipped upside down into a new tube to collect DNA and centrifuged for $3 \mathrm{~min}$ at 4,000 g. $\sim 500$ $\mu \mathrm{L}$ of DNA was recovered. RNA was degraded by adding $1 \mu \mathrm{L}$ of $10 \mathrm{mg} / \mathrm{mL}$ RNAase $A$ and incubation for $30 \mathrm{~min}$ at $37^{\circ} \mathrm{C}$. The amount of DNA was estimated by running an aliquot on a $1 \%$ Agarose gel along with a $1 \mathrm{~kb}$ ladder (NEB\#N3232s).

Biotin Fill-in: 1XNEB3.1 was added the reaction to a final volume of $680 \mu \mathrm{L}$, and then the 680 $\mu \mathrm{L}$ was split over $21.5 \mathrm{~mL}$ tubes. DNA ends were filled in and marked with biotin-14-dATP. To each tube $60 \mu \mathrm{L}$ of biotin fill-in master mix was added: [1xNEB2.1, $0.25 \mathrm{mM} \mathrm{dCTP}, 0.25 \mathrm{mM}$ dGTP, $0.25 \mathrm{mM}$ dTTP, $0.25 \mathrm{mM}$ biotin-dATP (ThermoFisher\#19524016), $50 \mathrm{U}$ Klenow polymerase Polymerase I (NEB M0210L)]. Samples were incubated at $37^{\circ} \mathrm{C}$ in a Thermocycler for 75 mins. Next, the tubes were placed on ice immediately for $15 \mathrm{mins}$, and samples from the 2 tubes were combined to obtain a final volume $\sim 800 \mu \mathrm{L}$. Amicon filters were used to reduce the volume of the final sample from $801 \mu \mathrm{L}$ to $130 \mu \mathrm{L}$.

Sonication: DNA was sonicated to a size of $100-300$ bp using a Covaris instrument (Duty Cycle 10\%, Intensity 5, Cycles per Burst 200, set Mode Frequency sweeping, continuous degassing, process time $60 \mathrm{sec}$, Number of cycles) for 4 cycles. The $130 \mu \mathrm{L}$ of sonicated DNA was transferred to a $1.5 \mathrm{~mL}$ tube and 1 XTLE was added to a total volume of $500 \mu \mathrm{L}$. DNA fragment size was determined by running $2 \mu \mathrm{L}$ of DNA along with low molecular ladder (NEB) on a $2 \%$ agarose gel.

Size fractionation using AMpure XP: $500 \mu \mathrm{L}$ AMpure beads (Beckman Coulter A63881) were added to a $1.5 \mathrm{~mL}$ tube labeled as $1.1 \mathrm{X}$. The tube was placed on the MPS for $5 \mathrm{~min}$, and the supernatant was removed. Beads were resuspended in $150 \mu \mathrm{L}$ AMpure mixture in order to make 1.1X. $400 \mu \mathrm{L}$ of AMpure mixture was added to $500 \mu \mathrm{L}$ of sonicated DNA from the previous step and the tube was labeled $0.8 \mathrm{X}$. The sample was vortexed and centrifuged briefly using a tabletop small centrifuge followed by incubation at RT for $10 \mathrm{~min}$ on a rocking platform. Then the 
tube was placed on the MPS for $5 \mathrm{~min}$ at RT. The $0.8 \mathrm{X}$ supernatants were collected and added to the 1.1X tube, the tube was briefly vortexed and centrifuged followed by incubation at RT for $10 \mathrm{~min}$ on a rocking platform. The tube was placed on the MPS for $5 \mathrm{~min}$ at RT, and the supernatant discarded. Beads in the $0.8 \mathrm{X}$ and $1.1 \mathrm{X}$ tubes were washed twice with $1 \mathrm{~mL} 70 \%$ ethanol, reclaiming beads against the MPS for $5 \mathrm{~min}$. Beads on the MPS were then dried until ethanol had evaporated completely. Next, $51 \mu \mathrm{L}$ of 1 XTLE was added to the $0.8 \mathrm{X}$ and $1.1 \mathrm{X}$ tubes to elute DNA from the beads. Tubes were incubated at RT on a rocking platform for 10 min. The $0.8 X$ and $1.1 X$ tubes were placed on the MPS for 5 min. Finally, the supernatants were transferred to $1.7 \mathrm{~mL}$ tubes labeled $0.8 \mathrm{X}$ and $1.1 \mathrm{X}$. The $1.1 \mathrm{X}$ sample contains DNA that ranges in size from $100-300 \mathrm{bp}$. The DNA in the $0.8 \mathrm{X}$ sample was kept in case more DNA was required, in which case the DNA would be sonicated using 2 cycles followed by a similar round of size fractionation as described above. The amount of DNA from both samples $0.8 \mathrm{X}$ and $1.1 \mathrm{X}$ was quantified by running $1 \mu \mathrm{L}$ on a $2 \%$ agarose gel along with a titration of low molecular weight DNA ladder (100 ng, $200 \mathrm{ng}, 400 \mathrm{ng}$ ).

End Repair: $50 \mu \mathrm{L}$ from the 1.1X sample was transferred to a PCR tube, and $20 \mu \mathrm{L}$ of end repair mix was added: [3.5X NEB ligation buffer (NEB B0202S), $0.875 \mathrm{mM}$ dNTP mix, 0.375 $\mathrm{U} / \mu \mathrm{L}$ T4 DNA polymerase, $1.25 \mathrm{U} / \mu \mathrm{L}$ T4 polynucleotide kinase, $0.125 \mathrm{U} / \mu \mathrm{L}$ Klenow DNA polymerase]. The $70 \mu$ total volume reaction was incubated for $30 \mathrm{~min}$ at $20^{\circ} \mathrm{C}$ in a PCR machine and then placed on ice. The DNA was purified by $1: 2$ Ampure, by adding $140 \mu \mathrm{L} 2 \mathrm{X}$ Ampure solution to the $70 \mu \mathrm{L}$ DNA sample followed by incubation for 5 min at RT. The tube was placed on the MPS for 4 min to reclaim the beads and the supernatant was discarded. The beads were washed twice with $1 \mathrm{~mL}$ of $70 \%$ ethanol while on the MPS. After beads were dried DNA was eluted in $32 \mu \mathrm{L}$ TLE (pH 8.0) and incubation for 10 min at RT. The supernatant was transferred to a $1.5 \mathrm{~mL}$ tube.

A-tailing: A dATP was added to the 3' ends by adding $18 \mu \mathrm{L}$ of A-tailing mix [5 $\mu \mathrm{L}$ NEB buffer $3.1,10 \mu \mathrm{L}$ of $1 \mathrm{mM}$ dATP, $3 \mathrm{U}$ Klenow exo (NEB M0212S)] to the $32 \mu \mathrm{L}$ of DNA sample from the previous step. The reaction was then incubated in a PCR machine at $37^{\circ} \mathrm{C}$ for 30 min followed by incubation $65^{\circ} \mathrm{C}$ for $20 \mathrm{~min}$ and cooling down to $4^{\circ} \mathrm{C}$. The tube was placed on ice. The volume was brought to $100 \mu \mathrm{L}$ by adding $1 \mathrm{X}$ NEB2.1. The DNA was then purified by adding 1:2 Ampure mix ( $200 \mu \mathrm{L}$ of Ampure was added to the $100 \mu \mathrm{L}$ final DNA volume). Finally, the DNA was eluted in $40 \mu \mathrm{L}$ of $1 \mathrm{X}$ T4 DNA ligase buffer (Invitrogen $5 \mathrm{X}$ ).

Illumina adapter ligation and paired-end PCR: For this step we used the TruSeq DNA LT kit Set A (REF\#15041757). $50 \mu \mathrm{L}$ of ligation mix [25 $\mu \mathrm{L}$ Illumina paired-end adapters, $15 \mu \mathrm{L}$ T4 DNA ligase Invitrogen, $10 \mu \mathrm{L}$ 5X T4 DNA ligase buffer (Invitrogen 5X)] was added to the $40 \mu \mathrm{L}$ sample from the previous step. The ligation sample was then incubated at RT for 2 hours on a rotator. Next, the DNA was purified by adding 1:1 Ampure solution (180 $\mu \mathrm{L}$ of Ampure mix was added to the $90 \mu \mathrm{L}$ sample), the supernatant was discarded and beads were washed twice with $1 \mathrm{~mL}$ of $70 \%$ ethanol. After the last wash step, the beads were resuspended in $400 \mu \mathrm{L}$ of $1 \mathrm{X}$ TLE and incubated at RT on a rocking platform for 10 mins. The tube was placed on the MPS for 4 mins. Finally, the $400 \mu \mathrm{L}$ supernatant was transferred to a new tube.

Biotin pull-down: All the following steps are done using $1.5 \mathrm{~mL}$ loBind tube (Eppendorf 22431021). $15 \mu \mathrm{L}$ of MyOne streptavidin C1 beads mix (Thermo Fisher 65001) was transferred to a $1.5 \mathrm{~mL}$ tube. The beads were washed twice with $400 \mu \mathrm{L}$ of TWB [5 mM Tris- $\mathrm{HCl} \mathrm{pH} 8.0,0.5$ $\mathrm{mM}$ EDTA, $1 \mathrm{M} \mathrm{NaCl}, 0.05 \%$ Tween20] by incubation for $3 \mathrm{~min}$ at RT. After each wash, the tube 
was placed on the MPS for 1 min and the supernatant was removed. After the washes, the beads were resuspended in $400 \mu \mathrm{L}$ of $2 X$ Binding Buffer (BB) $[10 \mathrm{mM}$ Tris- $\mathrm{HCl} \mathrm{pH} 8.0,1 \mathrm{mM}$ EDTA, $2 \mathrm{M} \mathrm{NaCl}$ ] and mixed with the $400 \mu \mathrm{L}$ DNA from the previous step in a new $1.5 \mathrm{~mL}$. The mixture was incubated for $15 \mathrm{~min}$ at RT with rotation. The tube was then placed on the MPS for 1 min and the supernatant was removed. The DNA bound to the beads was washed first by adding $400 \mu \mathrm{L}$ of $1 \mathrm{X} \mathrm{BB}$ and transferring to a new tube. The beads were reclaimed against the MPS for $1 \mathrm{~min}$, and the supernatant discarded. $100 \mu \mathrm{L}$ of $1 \mathrm{X}$ TLE was added and the beads were reclaimed against the MPS for $1 \mathrm{~min}$, then the supernatant was discarded. Finally, the DNA bound to the beads was eluted in $32.5 \mu \mathrm{L}$ of $1 \mathrm{X}$ TLE.

PCR optimization: The Illumina Truseq Kit (DNA LT kit Set A (REF\#15041757)) was used for PCR amplification of DNA for Dpnll-Seq. The trial PCR reaction was set up as follows: $[2.5 \mu \mathrm{L}$ DNA bound to beads, $2 \mu \mathrm{L}$ of Primers mix (Truseq kit), $10 \mu \mathrm{L}$ Master Mix (Truseq kit), $10.5 \mu \mathrm{L}$ of ultrapure distilled water (Invitrogen)]. The $25 \mu \mathrm{L}$ was split over four PCR tubes (5 $\mu \mathrm{L} /$ per tube). Each of the four samples was incubated for different PCR cycles $(6,8,10$, or 12 cycles): [30 sec at $98^{\circ} \mathrm{C}$, n cycles of $\left(30 \mathrm{sec}\right.$ at $98^{\circ} \mathrm{C}, 30 \mathrm{sec}$ at $65^{\circ} \mathrm{C}, 30 \mathrm{sec}$ at $\left.72^{\circ} \mathrm{C}\right), 7 \mathrm{~min}$ at $72^{\circ} \mathrm{C}$, hold at $10^{\circ} \mathrm{C}$. The optimal PCR cycle number needed to get enough DNA for sequencing was determined by running the 4 PCR reactions on a $2 \%$ agarose gel along with low molecular ladder titration (100 ng, $200 \mathrm{ng}, 400 \mathrm{ng}$ ). Three PCR reactions of $50 \mu \mathrm{L}$ volume were then performed: [ $5 \mu \mathrm{L}$ DNA bound to beads, $4 \mu \mathrm{L}$ of Primers mix (Truseq kit), $20 \mu \mathrm{L}$ Master Mix ( Truseq kit), $21 \mu \mathrm{L}$ of ultrapure distilled water (Invitrogen)]. The 3 PCR reactions were pooled together to obtain $150 \mu \mathrm{L}$ total volume. The samples were reclaimed against the MPS for $1 \mathrm{~min}$, then the PCR products (supernatant) were taken to new $1.5 \mathrm{~mL}$ tubes. 1:1 Ampure was performed for removal of primer dimers (150 $\mu \mathrm{L}$ of Ampure and $150 \mu \mathrm{L}$ DNA sample). Finally, beads were resuspended in $35 \mu \mathrm{L}$ of TLE to elute the DNA. DNA that remained bound to beads was saved after a first wash using TBW followed by two washes with 1 X TLE and then resuspended in $30 \mu \mathrm{L}$ of $1 \mathrm{X}$ TLE.

\section{Lamin A Immunofluorescence and DAPI}

For nuclei immunofluorescence, we prepared a coverslip by adding $1 \mathrm{~mL}$ of $0.1 \%$ Poly-L-lysine solution (Sigma SLBQ5716V) for $10 \mathrm{~min}$, then coverslips were dried using Whatman papers. Each coverslip was transferred to a single well of an eight wells plate. The coverslips were washed twice using PBS. Next $500 \mu \mathrm{L}$ of $30 \%$ sucrose with $1 \mathrm{mM}$ DTT was added on top of the coverslips to protect nuclei from an abrupt contact with coverslip during spinning. 1 million control nuclei or nuclei after chromatin digestion were crosslinked for 20 min using a $4 \%$ final concentration of Paraformaldehyde immediately after pre-digestion. Next, nuclei were added slowly on top of he sucrose solutions on the coverslips and spun for 15 mins at $2,500 \mathrm{~g}$ at $4^{\circ} \mathrm{C}$. Next, nuclei were assumed to be attached to the coverslips which were then transferred to a new 8 well plate. The coverslips were washed five times with $1 \%$ PBS. Next, non-specific binding of the primary antibody was blocked by adding $500 \mu \mathrm{L}$ of the blocking buffer [3\% BSA, 1X PBS, 0.1\% Triton X-100 (Sigma 9002-93-1)] and incubating for $60 \mathrm{~min}$ at RT. Afterward, lamin $A$ antibody (ab 26300) was diluted 1:1000 in blocking buffer, and the coverslip was incubated face-down on top of a $250 \mu \mathrm{L}$ of lamin A antibody droplet that was placed on parafilm for 120 min at RT. Then, the coverslip was placed back in the well of a new plate face-up and washed five times with washing buffer (1X PBS, 0.1\% Triton X-100). The secondary antibody 
Goat Anti-Rabbit (ab150077) was diluted 1:1000 in blocking buffer, and the coverslip was incubated face-down on top of a $250 \mu \mathrm{L}$ droplet of the secondary antibody (Goat Abti-Rabbit (ab150077) that was placed on parafilm for 60 min at RT. Next, the coverslip was placed back in the well of a new plate face-up and washed five times with washing buffer (1X PBS, $0.1 \%$ Triton $\mathrm{X}-100)$ and twice with $1 \mathrm{X}$ PBS. The slide was mounted and sealed using $10 \mu \mathrm{L}$ antifade mountant with DAPI (Invitrogen P36931).

For image acquisition, we used a Nikon Eclipse Ti microscope. Imaging was performed using an Apo TIRF, N.A. 1.49, 60X oil immersion objective (Nikon), and a Zyla sCMOS camera (Andor). Z-series of $0.2 \mu \mathrm{m}$ slices were acquired using Nikon Elements software (Version 4.4).

\section{Chromatin fractionation assay}

Chromatin-bound proteins were isolated and separated from free proteins. A sample of 2 million control nuclei or pre-digested nuclei (obtained as described above "Pre-digestion of nuclei") was centrifuged at $5,000 \mathrm{~g}$ for $5 \mathrm{~min}$ at $4^{\circ} \mathrm{C}$. The supernatant was transferred to an Amicon column to reduce the volume from $500 \mu \mathrm{L}$ to $100 \mu \mathrm{L}$ by centrifugation for $4 \mathrm{~min}$ at $14,000 \mathrm{~g}$. This sample contains the free protein fraction. Next, $26 \mu \mathrm{L}$ of glycerol and $1.3 \mu \mathrm{L}$ of $100 \mathrm{X}$ protease inhibitor cocktail were added to the $100 \mu \mathrm{L}$ free proteins sample. The pellet containing the nuclei was resuspended in $100 \mu \mathrm{L}$ of nuclei purification buffer with Triton (10 mM PIPES pH 7.4, $10 \mathrm{mM}$ $\mathrm{KCl}, 2 \mathrm{mM} \mathrm{MgCl} 2,0.25 \%$ Triton, $1 \%$ Protease inhibitor, $1 \mathrm{mM} \mathrm{DTT}$ ) and incubated for $10 \mathrm{~min}$ on ice. Then, in order to protect protein structure during sonication, $25 \mu \mathrm{L}$ of glycerol was added to the $100 \mu \mathrm{L}$ pellet sample to have $20 \%$ final glycerol concentration. The sample was sonicated using a Covaris instrument at $4^{\circ} \mathrm{C}$ as follows: (Duty Cycle 10\%, Intensity 5, Cycles per Burst 200 , set Mode Frequency sweeping, continuous degassing, process time $60 \mathrm{sec}, 4$ cycles). The pellet sample contains chromatin-bound proteins, was transferred to a $1.5 \mathrm{~mL}$ tube. All samples were stored at -20 . These samples contain the protein bound CTCF and cohesin. Note: when these samples were centrifuged after the triton solubilization, we found that no SMC3 or CTCF could be detected in the supernatant. These results indicate that non-chromatin-bound proteins exit the nuclei and were recovered in the supernatant prior to triton solubilization step.

For analysis of CTCF and SMC1 chromatin binding: $15 \mu \mathrm{L}$ from each protein sample (supernatant or pellet) was mixed with $5 \mu \mathrm{L}$ of $5 \mathrm{X}$ Lane Marker Reducing Sample Buffer (Thermo Fisher 39000), then the mix was boiled for $10 \mathrm{~min}$. The samples were cooled down to RT before loading them on a 3-8\% Tris-Acetate Protein Gels (Invitrogen EA0375PK2). Next, the gel was run in 1X Tris-Acetate SDS Running Buffer (Invitrogen LA0041) for 75 min at 150V. For Histone H3: $1 \mu \mathrm{L}$ of protein sample was mixed with $14 \mu \mathrm{L}$ of PBS containing $1 \%$ Protease inhibitor, $5 \mu \mathrm{L}$ of $5 \mathrm{X}$ Lane Marker Reducing Sample Buffer was added to the mix and boiled for $10 \mathrm{~min}$. The samples were cooled down to RT before loading them in Tris-Base $4-12 \%$ (Invitrogen NP0322BOX), then the gel was run in 1X MES-SDS running buffer (Invitrogen $\mathrm{B} 0002$ ) for $60 \mathrm{~min}$ at $150 \mathrm{~V}$. The proteins were transferred from the gel to nitrocellulose membrane using $1 \mathrm{X}$ western blot transfer buffer (Thermo science 35040). The transfer was 120 min for SMC1 and CTCF and 75 min for $\mathrm{H} 3$. The nitrocellulose membranes were washed using 1X TBST [50 mM Tris-Cl, pH 7.6; $150 \mathrm{mM} \mathrm{NaCl}, 0.1 \mathrm{~mL}$ of Tween 20], then Blocked for $120 \mathrm{~min}$ using $5 \%$ milk ( $1 \mathrm{~g}$ milk in $20 \mathrm{~mL} 1 \mathrm{X}$ TBST). The membrane when then incubated overnight at $4{ }^{\circ} \mathrm{C}$ with primary antibody diluted in $5 \%$ milk [1:1000 CTCF antibody cell signaling (activeMotif 
61311), 1:2000 SMC1 (Bethyl Antibody, A300-055A), 1:4000 H3 Abcam (ab1791)] . Next, the membranes were washed 6 times for 10 min per wash using 1X TBST. The secondary antibody anti-rabbit lgG HRP from cell signaling was diluted using 5\% milk for CTCF and SMC1 [1:1000 for CTCF, 1:2000 SMC1] and in 1\% milk for H3 1:5000 dilution. Membranes were incubated for 120 min at RT. Finally, membranes were washed 6 times for 10 min using 1 X TBST. Finally, the membranes were developed using luminol-based enhanced chemiluminescence(Thermo science 34076).

\section{Micromanipulation force measurement and treatments of an isolated nuclei} Micromanipulation force measurements were conducted as described previously in Stephens et al. (Stephens et al., 2017). K562 cells were grown in microscope slide wells and treated with 1 $\mu \mathrm{g} / \mathrm{mL}$ latrunculin A (Enzo Life Sciences) for $\sim 45 \mathrm{~min}$ before single nucleus isolation. The nucleus was isolated by using small amounts of detergent (0.05\% Triton X-100 in PBS) locally sprayed onto a living cell via an "isolation" micropipette. This gentle lysis allows the use of a second micropipette to retrieve the nucleus from the cell, using slight aspiration and non-specific adherence to the inside of the micropipette. A third micropipette was then attached to the opposite end of the nucleus in a similar fashion. This last "force" micropipette was pre-calibrated for its deflection spring constant, which is on the order of $2 \mathrm{nN} / \mu \mathrm{m}$. A custom computer program written in LabView was then run to move the "pull" micropipette and track the position of both the "pull" and "force" pipettes. The "pull" pipette was instructed to move $5 \mu \mathrm{m}$ at $45 \mathrm{~nm} / \mathrm{sec}$. The program then tracked the distance between the pipettes to provide a measure of nucleus extension $\sim 3 \mu \mathrm{m}$. Tracking the distance that the "force" pipette moved/deflected multiplied by the pre-measured spring constant provides a calculation of force exerted. Calculations were done in Excel (Microsoft) to produce a force-extension plot from which the best-fit slope of the line would provide a spring constant of the nucleus $(\mathrm{nN} / \mu \mathrm{m})$. Isolated nuclei were measured twice initially to establish the native spring constant prior to treatment. After $50 \mathrm{uL}$ of buffer only (control), 100 units DpnII (|GATC) with NEB buffer 3.1, or 100 units HindIII (A|AGCTT) with NEB buffer 2.1 was added to the $1.5 \mathrm{~mL}$ imaging well and mixed gently. Force measurements were performed $5 \mathrm{~min}, 30 \mathrm{~min}$, and $60 \mathrm{~min}$ post-treatment.

\section{QUANTIFICATION AND STATISTICAL ANALYSIS}

\section{C-PCR}

The human $\beta$-globin locus is an ideal region to examine looping interactions between enhancers and genes because of the strong looping interactions between the LCR and HBG globin gene in the erythroleukemia cell line K562, which highly expresses the globin genes (Dostie et al., 2006). 3C libraries were generated from: (1) K562 cells that have an LCR-HBG interaction, (2) GM12878 cells in which the LCR-HBG looping interaction is absent, and (3) beta-globin BAC (ENm009) control to normalize for primer bias. To investigate the interaction between the LCR and HBG gene, 3C primers from (Dostie et al., 2006) were used. 16 forward primers of 28-33 bp length were designed 40-60 bp upstream of each EcoRI site throughout a $110 \mathrm{~kb}$ region around the Beta Globin locus (chr11: 5221788- 5337325). The EcoRI fragment overlapping with the LCR $(H S 3,4,5)$ was used as an anchor to detect the interaction frequencies between the LCR 
and EcoRI fragments throughout the $\beta$-globin locus. For each primer pair, triplicate PCR reactions were set up, and the mean of the three was normalized to the BAC signal for the same primer pair before plotting normalized interaction frequency in the $y$-axis, the distance from EcoRI fragment overlapping with LCR to neighboring EcoRI fragments is plotted in the $x$ axis. Error bars are the standard error of the mean (SEM). PCR primers are listed in Supplemental Table S1.

\section{C data processing}

The fastq files for $5 \mathrm{C}$ sequencing data were processed as described in https://github.com/dekkerlab/5C-CBFb-SMMHC-Inhib/blob/master/data_processing_steps.md The Fastq files were mapped using novoalign to a reference genome built from the pool of all 277 probes. After mapping, we combined the read-pairs. The results were then transferred to a matrix format, and interactions were filtered as previously described (Lajoie et al., 2009; Sanyal et al., 2012). First, interactions that belong to the same EcoRI fragment were removed. Second, outliers that are overrepresented as a result of overamplification were also removed. Outliers were defined as the interactions with a Z-score greater than 20 in all datasets. Third, probes that strongly over or underperform which leads to strongly enriched or depleted interactions in a whole row of interactions, were also removed. The four matrices were then scaled to the same number of total reads. Finally, data were binned at $20 \mathrm{~Kb}$ (median) with a sliding window with $2.5 \mathrm{~Kb}$ steps.

\section{Hi-C data processing}

$\mathrm{Hi}-\mathrm{C}$ read mapping, filtering, binning and matrix normalization were performed using the cMapping pipeline available at https://github.com/dekkerlab/cMapping (Lajoie et al., 2015). In brief, $\mathrm{Hi}-\mathrm{C}$ reads were mapped to reference human genome assembly hg19 using an iterative mapping strategy and Bowtie 2 (Langmead et al., 2009). Successfully mapped reads were then filtered to remove reads mapping to the same restriction fragment and to remove PCR duplicates. Interaction frequency versus distance plots displayed high variance for interactions . below $1 \mathrm{~kb}$ for all samples. Hence, after mapping of valid pair, we removed all pairs with a genomic distance less than $1 \mathrm{~kb}$. The remaining valid read pairs were then binned to $500 \mathrm{~kb}, 40$ $\mathrm{kb}$, and $10 \mathrm{~kb}$ resolution matrices. Outlier bins of these matrices with low signal were assigned values of NA. Then as a bias correction step, matrices were normalized such that the sum of interactions in each row/column are approximately equivalent via an iterative correction procedure (ICE) (Imakaev et al., 2012). Lastlly, for comparison between samples, matrices were scaled such that the total interactions for a genome-wide matrix equals one billion for each sample. These ICEd scaled matrices were used for subsequent analyses.

\section{A/B compartments}

All reads from $\mathrm{Hi}-\mathrm{C}$ in control K562 samples were pooled to identify $\mathrm{A}$ (active) and $\mathrm{B}$ (inactive) compartments in K562 cells. A/B compartments were identified at $40 \mathrm{~kb}$ resolution following the procedure described in (Lieberman-Aiden et al., 2009) using matrix2compartment.pl in https://github.com/dekkerlab/cworld-dekker. Briefly, each cis interaction matrix was first transformed into a z-score matrix followed by transformation into a correlation matrix. PCA was 
performed on the correlation matrix and the first eigenvector (PC1) of the PCA analysis was used to identify compartments for each chromosome. A/B compartments were assigned based on gene density such that the A-compartment was more gene-dense than the B-compartment. Positive PC1 values indicate gene-rich A compartments and negative PC1 values indicate gene-poor B compartments. For chromosome 9 the compartments were called for each chromosome arm separately as PC1 captured preferences for interactions within the same arm as opposed to canonical compartment preferences.

\section{LOS and half-life calculation}

To measure the 3D structure changes resulting from Dpnll or HindIII pre-digestion we quantified the amount of cis interactions lost or gained in a $6 \mathrm{Mb}$ window centered at every $40 \mathrm{~kb}$ bin genome wide. For each $40 \mathrm{~kb}$ bin, the percent of interactions occurring within its $6 \mathrm{Mb}$ window (corresponding to interactions less than or equal to $3 \mathrm{Mb}$ in distance either upstream or downstream from $40 \mathrm{~kb}$ bin) out of total interactions for the $40 \mathrm{~kb}$ bin (cis and trans) was calculated. These $6 \mathrm{Mb}$ cis percentages were calculated for control, Dpnll pre-digested, and HindIII-pre-digested nuclei. The change in 3D structure relative to control using these cis percentages was given by the following loss of structure (LOS) metric:

$$
\text { LOS }=\frac{\text { Control }_{\text {Cis } \%}-\text { Predigest }_{\text {Cis } \%}}{\text { Control }_{C i S} \%}
$$

Hence, LOS values in the range $(0,1)$ represent a loss in short range contacts after predigestion; LOS values < 0 represent an increase in short range contacts after pre-digest, and an LOS equal to zero would indicate no change in structure after pre-digestion. A window of $6 \mathrm{Mb}$ was chosen as we sought here to quantify interactions disrupted by pre-digestion. Many longer range interactions increased after pre-digestion, potentially due to random ligations of cut fragments that start to mix. Differences noted in A and B stability was preserved when LOS was calculated using cis percents for entire chromosomes as opposed to a $6 \mathrm{Mb}$ window, however the size of chromosomes did bias results by giving $40 \mathrm{~kb}$ bins in small chromosomes greater LOS.

To quantify the timing of disrupted interactions we generated a half-life track utilizing the Hi-C matrices from the Dpnll timecourses. For each $40 \mathrm{~kb}$ bin we fit a curve to the LOS of each timepoint following an exponential decay of the form (Supplemental Figure 4C):

$$
\text { LOS }=a-\left(b \times e^{-c \times \text { minutes }}\right)
$$

such that $a, b$ and $c$ are parameters to fit. The half-life, $\mathrm{t}_{1 / 2}$, was defined as the time required to reach half saturation, saturation being the 16 hour timepoint where maximal cis interactions have been lost. Half-life values were then computed for every $40 \mathrm{~kb}$ bin genome wide. To remove noisy and less reliable $t_{1 / 2}$ data, we first removed all extreme outliers bins where the sum of squared residuals (SSR) for the exponential fit was greater than 0.1 . Then all bins with an 
SSR greater than two standard deviations from the mean was deemed an outlier and also removed from analyses.

As LOS and $t_{1 / 2}$ are both dependent on digestion efficiency we also generated residual LOS and $t_{1 / 2}$ tracks to account for bin to bin variation in digestion efficiency. We used a moving average approach to calculate residuals for LOS as a function of Dpnll-seq signal and also $t_{1 / 2}$ as a function of DpnIl-seq signal since the relationships between these variables were non-linear (Figure 3F left, Supplementary Figure $5 \mathrm{C}$ ). For both stability metrics LOS and $\mathrm{t}_{1 / 2}$, a sliding window of 200 Dpnll-seq signal with a step size of one was used to calculate mean LOS or $t_{1 / 2}$ signal for each Dpnll-seq signal increment (Figure 3F left, Supplementary Figure 5C). Window and step size were selected by manual inspection of moving averages and compromising between over and underfitting. These moving averages were used to calculate residuals such that a positive LOS residual indicates more structure loss than expected by given digestion efficiency and a negative LOS residual indicates less structure loss than expected. As $t_{1 / 2}$ is inversely related to LOS, positive $t_{1 / 2}$ residuals indicate less structure loss than expected and negative $t_{1 / 2}$ residuals indicate more structure loss than expected. Moving averages were also used to generate residuals for Dpnll-seq as a function of PC1 and LOS as a function of PC1 (Figure 3G, right)

\section{Dpnll-seq data analysis}

Sequenced reads were mapped to the genome using the Bowtie read aligner (Langmead et al., 2009) and reads mapping to multiple sites of the genome were removed. As expected, a high percentage of reads mapped precisely to their associated restriction cut site (Supplementary Figure $3 \mathrm{C}$ ). To remove potential artificial biases, we filtered out paired-end reads from fragments whose start or end coordinate was more than three nucleotides from an appropriate restriction cut site. Filtered reads were then binned to $500 \mathrm{~kb}$ or $40 \mathrm{~kb}$ resolutions. The K562 cell line has a primarily triploid karyotype with regions of the genome in diploid and tetraploid states. Copy number state assignments for each $500 \mathrm{~kb}$ or $40 \mathrm{~kb}$ bin were assigned using publicly available K562 copy number data from the Catalogue of Somatic Mutations In Cancer (COSMIC) database (https://cancer.sanger.ac.uk/cell lines/download). Copy number segments in the COSMIC dataset were identified by PICNIC analysis of Affymetrix SNP6.0 array data (PMID:19837654). Read coverage files at $500 \mathrm{~kb}$ and $40 \mathrm{~kb}$ were corrected to a genome wide diploid state using the copy number state assignments and dividing coverage by appropriate correction value (diploid $=1$, triploid $=1.5$, tetraploid $=2$, etc.) per bin. (Supplementary Figure $3 D, E)$. Final copy number corrected coverage files were used for all downstream analysis and are available (Supplementary File). Dpnll-seq computational workflow is maintained at https://github.com/tborrman/Dpnll-seq

\section{Subcompartments}

Rao et al. (2014) divided the canonical A/B compartments into five primary subcompartments A1, A2, B1, B2, B3 based on each subcompartment's preferential Hi-C interactions in GM12878 cells. Subcompartments were annotated using high resolution $(\sim 1 \mathrm{~kb}) \mathrm{Hi}-\mathrm{C}$ data and were shown to display unique genomic and epigenomic profiles. K562 subcompartments were annotated in (Xiong and Ma, 2019) via the method SNIPER using lower resolution Hi-C data. In 
short, SNIPER infers subcompartments via a neural network approach to accurately annotate subcompartments using $\mathrm{Hi}-\mathrm{C}$ datasets with moderate coverage ( 500 million mapped read pairs). Xiong et al.'s K562 SNIPER subcompartments showed a substantial conservation with GM12878 annotations from Rao et al. (Rao et al., 2014) and were also enriched in similar epigenetic features, hence we utilized these SNIPER annotations to compare subcompartment status with chromatin stability. K562 SNIPER subcompartments were annotated at $100 \mathrm{~kb}$ resolution. To compare with our $40 \mathrm{~kb}$ resolution liquid chromatin $\mathrm{Hi}-\mathrm{C}$ data, we binned the 100 $\mathrm{kb}$ subcompartment annotations to $40 \mathrm{~kb}$ such that any $40 \mathrm{~kb}$ bin overlapping a boundary of two separate subcompartments was assigned a value of NA. Upon piling up K562 subcompartment boundaries, we also found enrichment and depletion of various chromatin features consistent with those described in both Rao et al. (Rao et al., 2014) and Xiong et al. (Xiong and Ma, 2019).

\section{Sub-nuclear structures}

To assess the effect of sub-nuclear structures on chromatin stability we utilized the extensive genetic and epigenetic data publicly available for K562 cells (Supplemental Table S3).

Fold change over control ChIP-seq tracks for histone modifications, chromatin remodellers, and other various proteins were downloaded from the ENCODE Portal. To compare ChIP-seq data with $t_{1 / 2}$, or residuals of $t_{1 / 2}$ after correction for Dpnll-signal, we binned the ChIP-seq signal tracks into $40 \mathrm{~kb}$ such that each $40 \mathrm{~kb}$ bin represented the mean signal found across the bin. Bins with no overlapping signal were designated a value of NA.

To examine the association between methylation state and $t_{1 / 2}$ or residuals of $t_{1 / 2}$ after correction for Dpnll-signal, we downloaded methylation state at CpG Whole-Genome Bisulfite Sequencing (WGBS) tracks from ENCODE. As the methylation data was mapped to hg38, we used the UCSC LiftOver program to convert coordinates to hg19. Then percentage methylation at CpG sites was binned to $40 \mathrm{~kb}$ resolution using the mean.

As there is currently no nucleolus associated domains (NADs) data available for K562, we analyzed a binary NADs state track for the human embryonic fibroblast IMR90 cell line (Dillinger et al., 2017). Dillinger et al. annotated NADs via a two-state hidden Markov model of aCGH data from DNA of isolated nucleoli. Using these annotated NADs, coverage of each $40 \mathrm{~kb}$ bin for NADs was assessed and used for all our downstream analyses.

Mapping of nuclear speckle, nuclear lamina and Polll associated loci for K562 cells was accomplished recently via the TSA-seq protocol (Chen et al., 2018). Signal tracks of log2(pulldown/input) were downloaded from GEO and binned to $40 \mathrm{~kb}$ as previously described for ChIPseq files. Microarray data for LaminB1 associated domains identified through the DamID protocol was also available from that study. We used the UCSC LiftOver program to convert coordinates from hg18 to hg19. We then binned the log2(Dam-LaminB1/Dam) signal to $40 \mathrm{~kb}$ bins as previously described for ChIP-seq files.

To analyze cell cycle relationship with chromatin stability we downloaded Repli-seq data for K562 cells from ENCODE. Actively replicating regions are quantified as a percentage 
normalized signal for FACS sorted cells in G1 phase, four stages of S phase (S1-S4) and G2 phase. Signal tracks for Repli-seq data were binned to $40 \mathrm{~kb}$ as previously described for ChIPseq files.

Binning of data was performed using the bedtools/v2.26.0 software. To assess the quality of the publicly downloaded data we generated the spearman correlation matrix of all binned signal tracks (Figure S6A). Hierarchical clustering of rows of the correlation matrix position heterochromatic marks (H3K9me3, HP1 $\alpha$, HP1 $\beta$, NADs, and LADs) near one another as expected. The majority active marks form a larger cluster, with the markers for polycomb regions (H3K27me3, CBX8, BMI1, RNF2, and SUZ12) representing facultative heterochromatin clustered together segregating active from inactive marks.

\section{Gene Expression}

To assess the effect of gene expression on chromatin stability we utilized processed gene expression quantifications of total RNA-seq for K562 cells available from ENCODE (Accession ID: ENCFF782PCD). Gene locations were mapped using the hg19 ensGene table from UCSC Table Browser. To compare expression values with $40 \mathrm{~kb}$ resolution $t_{1 / 2}$ or residuals of $t_{1 / 2}$ after correction for Dpnll-signal tracks, fragments per kilobase million (FPKM) values for each gene were binned to $40 \mathrm{~kb}$ such that each $40 \mathrm{~kb}$ bin represented the mean FPKM for all genes overlapping that bin. Bins without any genes were assigned a value of NA. Binned FPKM >=1 was determined to be a reasonable cutoff for expression by inspection of the full distribution of FPKM values.

\section{Compartmentalization saddle plots}

Saddle plot was adapted from cool tools (https://github.com/hms-dbmi/hic-data-analysisbootcamp/tree/master/notebooks/04_analysis_cooltools-eigenvector-saddle.ipynb).

To measure the strength of compartments the average intra-chromosomal interactions frequencies between $40 \mathrm{~kb}$ bins were normalized by genomic distance (observed/expected Hi-C maps). Then, in a 50 by 50 bin matrix, the distance corrected interaction bins were sorted based on their PC1 value in increasing order. Finally, all intra-chromosomal interactions with similar PC1 values were aggregated to obtain compartmentalization saddle plots. In these plots, preferential B-B interactions are in the upper left corner, and preferential A-A interactions are in the lower right corner.

\section{Homotypic interaction saddle plots}

The average intra-chromosomal interactions frequencies between $40 \mathrm{~kb}$ bins were normalized by genomic distance (observed/expected Hi-C maps). Then, in a 50 by 50 bin matrix, the distance corrected interaction bins were sorted based on their signal value (TSA-seq, DamID) for a given factor (SON, Lamin). Finally, all intra-chromosomal interactions with similar signal values were aggregated to obtain homotypic interaction saddle plots. In these plots, pair-wise interactions between loci enriched in factor binding are shown in the lower right corner, and pairwise interactions between loci not bound by the factor are shown in the upper left corner. 


\section{Scaling plot}

The script to generate scaling plots was adapted from cooltools (https://github.com/mirnylab/cooltools/tree/master/cooltools). Genome-wide curves of normalized contact frequency $P(s)$ is plotted as a function of genomic distance for all intrachromosomal interactions. Each library was normalized by total valid interactions

\section{Mean z-score heatmap}

Each genome wide $40 \mathrm{~kb}$ signal vector for a sub-nuclear structure was cleaned for outliers above three standard deviations of the vector's mean. Each cleaned vector was z-score transformed and then partitioned based on the different $t_{1 / 2}$ residual intervals for associated bins. The mean $z$-score for all bins within a given $t_{1 / 2}$ residual interval is plotted as a square in the heatmap.

\section{References}

Belaghzal, H., Dekker, J., and Gibcus, J.H. (2017). Hi-C 2.0: An optimized Hi-C procedure for high-resolution genome-wide mapping of chromosome conformation. Methods 123, 56-65. Chen, Y., Zhang, Y., Wang, Y., Zhang, L., Brinkman, E.K., Adam, S.A., Goldman, R., van Steensel, B., Ma, J., and Belmont, A.S. (2018). Mapping 3D genome organization relative to nuclear compartments using TSA-Seq as a cytological ruler. J Cell Biol 217, 4025-4048. de Gennes, P.-G. (1979). Scaling theory of polymer physics (Cornell University Press). Dillinger, S., Straub, T., and Nemeth, A. (2017). Nucleolus association of chromosomal domains is largely maintained in cellular senescence despite massive nuclear reorganisation. PLoS One 12, e0178821.

Dostie, J., Richmond, T.A., Arnaout, R.A., Selzer, R.R., Lee, W.L., Honan, T.A., Rubio, E.H., Krumm, A., Lamb, J., Nusbaum, C., et al. (2006). Chromosome Conformation Capture Carbon Copy (5C): A Massively Parallel Solution for Mapping Interactions between Genomic Elements. Genome Res 16, 1299-1309.

Ferraiuolo, M.A., Sanyal, A., Naumova, N., Dekker, J., and Dostie, J. (2012). From cells to chromatin: capturing snapshots of genome organization with $5 \mathrm{C}$ technology. Methods 58, 255267.

Imakaev, M., Fudenberg, G., McCord, R.P., Naumova, N., Goloborodko, A., Lajoie, B.R., Dekker, J., and Mirny, L.A. (2012). Iterative correction of Hi-C data reveals hallmarks of chromosome organization. Nature Methods 9, 999-1003.

Lajoie, B.R., Dekker, J., and Kaplan, N. (2015). The Hitchhiker's guide to Hi-C analysis: Practical guidelines. Methods 72, 65-75.

Lajoie, B.R., van Berkum, N.L., Sanyal, A., and Dekker, J. (2009). My5C: web tools for chromosome conformation capture studies. Nat Methods 6, 690-691.

Langmead, B., Trapnell, C., Pop, M., and Salzberg, S.L. (2009). Ultrafast and memory-efficient alignment of short DNA sequences to the human genome. Genome Biol 10, R25.

Leibler, L. (1980). Theory of microphase separation in block copolymers. Macromolecules 13, 1602-1617.

Lieberman-Aiden, E., van Berkum, N.L., Williams, L., Imakaev, M., Ragoczy, T., Telling, A., Amit, I., Lajoie, B.R., Sabo, P.J., Dorschner, M.O., et al. (2009). Comprehensive mapping of long-range interactions reveals folding principles of the human genome. Science 326, 289-293. Marko, J.F. (1993). Microphase separation of block copolymer rings. Macromolecules 26, $1442-$ 1444. 
Marko, J.F., and Siggia, E.D. (1997). Polymer models of meiotic and mitotic chromosomes. Mol Biol Cell 8, 2217-2231.

Marko, J.F., and Witten, T.A. (1991). Phase separation in a grafted polymer layer. Phys Rev Lett 66, 1541-1544.

Matsen, M.W., and Schick, M. (1994). Stable and unstable phases of a linear multiblcok copolymer melt. Macromolecules 27, 7157-7163.

Rao, S.S.P., Huntley, M.H., Durand, N.C., Stamenova, E.K., Bochkov, I.D., Robinson, J.T., Sanborn, A.L., Machol, I., Omer, A.D., Lander, E.S., et al. (2014). A 3D map of the human genome at kilobase resolution reveals principles of chromatin looping. Cell 159, 1665-1680. Sanyal, A., Lajoie, B.R., Jain, G., and Dekker, J. (2012). The long-range interaction landscape of gene promoters. Nature 489, 109-113.

Stephens, A.D., Banigan, E.J., Adam, S.A., Goldman, R.D., and Marko, J.F. (2017). Chromatin and lamin A determine two different mechanical response regimes of the cell nucleus. Mol Biol Cell 28, 1984-1996.

Xiong, K., and Ma, J. (2019). Revealing Hi-C subcompartments by imputing high-resolution inter-chromosomal interactions. BioRxiv doi: https://doi.org/10.1101/505503. 